\title{
The moduli space of regular stable maps
}

\section{Journal Article}

\section{Author(s):}

Robbin, Joel W.; Ruan, Yongbin; Salamon, Dietmar A.

Publication date:

2008

Permanent link:

https://doi.org/10.3929/ethz-b-000000359

Rights / license:

In Copyright - Non-Commercial Use Permitted

Originally published in:

Mathematische Zeitschrift 259(3), https://doi.org/10.1007/s00209-007-0237-x 


\title{
The moduli space of regular stable maps
}

\author{
Joel W. Robbin · Yongbin Ruan • Dietmar A. Salamon
}

Received: 15 March 2006 / Accepted: 17 June 2007 / Published online: 25 September 2007

C Springer-Verlag 2007

\begin{abstract}
We prove that the moduli space of regular stable maps in a complex manifold admits a natural complex orbifold structure. Our proof is based on Hardy decompositions and Fredholm intersection theory.
\end{abstract}

\section{Introduction}

This paper is a sequel to [4]. It studies the moduli space of stable maps whereas [4] studied the moduli space of stable marked nodal Riemann surfaces. The latter can be considered as a special case of the former by taking the target manifold $M$ to be a point. In both cases the moduli space is the orbit space of a groupoid where the objects are compact surfaces with additional structure. (We think of a map from a surface to another manifold as a structure on the surface.) In both cases the difficulty is that to achieve compactness of this moduli space it is necessary to include objects whose underlying surfaces are not homeomorphic.

Here we study only that part of the moduli space of stable maps which can be represented by regular stable maps. Only by restricting attention to regular stable maps can we hope to construct an orbifold structure. We also limit attention to target manifolds $M$ which are integrable complex and not just almost complex.

As in [4] we make heavy use of "Hardy decompositions". The idea is to decompose a Riemann surface $\Sigma$ into two surfaces $\Sigma^{\prime}$ and $\Sigma^{\prime \prime}$ intersecting in their common boundary $\Gamma$. A holomorphic map from $\Sigma$ into a complex manifold $M$ is uniquely determined by its

The authors would like to thank the referee for his/her diligent work. We are grateful for the careful attention to detail in the report.

J. W. Robbin · Y. Ruan

Mathematics Department, University of Wisconsin, Madison, WI 53706, USA

e-mail: robbin@math.wisc.edu

D. A. Salamon $(\bowtie)$

Department Mathematik, ETH Zürich, Rämistrasse 101, 8092 Zürich, Switzerland

e-mail: salamon@math.ethz.ch 
restriction to $\Gamma$ and so the space of all such holomorphic maps can be embedded into the space $\mathcal{V}$ of smooth maps from $\Gamma$ to $M$. In this way we identify the holomorphic maps with $\mathcal{V}^{\prime} \cap \mathcal{V}^{\prime \prime}$ where $\mathcal{V}^{\prime}$ and $\mathcal{V}^{\prime \prime}$ are the maps from $\Gamma$ to $M$ which extend holomorphically to $\Sigma^{\prime}$ and $\Sigma^{\prime \prime}$ respectively. (In the case where $\Sigma$ is the Riemann sphere, $M=\mathbb{C} \cup\{\infty\}$, and $\Gamma$ is the equator, $\mathcal{V}^{\prime}$ would consist of those maps whose negative Fourier coefficients vanish and $\mathcal{V}^{\prime \prime}$ would consist of those maps whose positive Fourier coefficients vanish. Hence the name Hardy decomposition.) The importance of this construction becomes clear when we consider a parameterized family $\left\{\Sigma_{b}\right\}_{b \in B}$ of Riemann surfaces. By judiciously choosing the decomposition we can arrange that the one dimensional manifolds $\Gamma_{b}$ are all diffeomorphic, even though the manifolds $\Sigma_{b}^{\prime}$ are not all homeomorphic. Then we identify the various $\Gamma_{b}$ with a disjoint union $\Gamma$ of circles. Under suitable hypotheses we are able to represent the holomorphic maps from $\Sigma_{b}$ to $M$ (for varying $b$ ) as a submanifold of the manifold of smooth maps from $\Gamma \cong \partial \Sigma_{b}^{\prime}=\partial \Sigma_{b}^{\prime \prime}$ to $M$.

Our theorems led to a theory of Fredholm triples in Sect. 6. These are triples $\left(X, X^{\prime}, X^{\prime \prime}\right)$ where $X$ is a Hilbert manifold and $X^{\prime}, X^{\prime \prime}$ are Hilbert submanifolds such that $T_{x} X^{\prime} \cap T_{x} X^{\prime \prime}$ and $T_{x} X /\left(T_{x} X^{\prime}+T_{x} X^{\prime \prime}\right)$ are finite dimensional for every $x \in X^{\prime} \cap X^{\prime \prime}$. We prove a finite dimensional reduction theorem for morphisms of such triples. We hope this theory has separate interest.

In Sect. 8 we show that the orbifold topology is the same as the well known topology of Gromov convergence.

Naming the additional structures which occur in this paper as opposed to [4] caused us to exhaust the Latin and Greek alphabets. Accordingly we have changed notation somewhat. For example, the aforementioned decomposition $\Sigma=\Sigma^{\prime} \cup \Sigma^{\prime \prime}$ was $\Sigma=\Delta \cup \Omega$ in [4]. We also use the following notations

$$
\begin{aligned}
\mathrm{g} & :=\text { arithmetic genus of } \Sigma / \nu, \\
\mathrm{n} & :=\text { number of marked points, } \\
\mathrm{k} & :=\text { number of nodal points, } \\
\mathrm{a} & :=\text { complex dimension of } A, \\
\mathrm{~b} & :=\text { complex dimension of } B, \\
\mathrm{~m} & :=\text { complex dimension of } M .
\end{aligned}
$$

We have used the $\backslash$ maths font for these integers so that we can write $a \in A, b \in B$ for the elements. We will also use the symbol $d$ to denote a homology class in $H_{2}(M ; \mathbb{Z})$.

\section{Stable maps}

2.1 Throughout let $(M, J)$ be a complex manifold without boundary. A configuration in $M$ is a tuple $\left(\Sigma, s_{*}, v, j, v\right)$ where $\left(\Sigma, s_{*}, v, j\right)$ is a marked nodal Riemann surface (see [4, Sect. 3]) whose quotient $\Sigma / v$ is connected and $v: \Sigma \rightarrow M$ is a smooth map satisfying the nodal conditions

$$
\{x, y\} \in v \Longrightarrow v(x)=v(y) .
$$

Thus $v$ descends to the quotient $\Sigma / v$ and we write $v: \Sigma / v \rightarrow M$ for a smooth map $v: \Sigma \rightarrow M$ satisfying the nodal conditions. We say that the configuration has type $(\mathrm{g}, \mathrm{n})$ if the marked nodal surface $\left(\Sigma, s_{*}, v\right)$ has type $(\mathrm{g}, \mathrm{n})$ in the sense of [4, Definition 3.7] and that 
it has type $(\mathrm{g}, \mathrm{n}, \mathrm{d})$ if in addition the map $v$ sends the fundamental class of $\Sigma$ to the homology class $\mathrm{d} \in \mathrm{H}_{2}(M ; \mathbb{Z})$. The configurations form the objects of a groupoid; an isomorphism

$$
\phi:\left(\Sigma^{\prime}, s_{*}^{\prime}, v^{\prime}, j^{\prime}, v^{\prime}\right) \rightarrow\left(\Sigma, s_{*}, v, j, v\right)
$$

is an isomorphism $\phi: \Sigma^{\prime} \rightarrow \Sigma$ of the underlying marked nodal Riemann surfaces such that

$$
v^{\prime}=v \circ \phi \text {. }
$$

Given two nonnegative integers $\mathrm{g}$ and $\mathrm{n}$ and a homology class $\mathrm{d} \in \mathrm{H}_{2}(M ; \mathbb{Z})$ we denote by $\mathcal{B}_{\mathrm{g}, \mathrm{n}}(M, J)$ the groupoid of configurations of type $(\mathrm{g}, \mathrm{n})$ and by $\mathcal{B}_{\mathrm{g}, \mathrm{n}, \mathrm{d}}(M, J)$ the subgroupoid of configurations of type $(g, n, d)$.

2.2 The configuration $\left(\Sigma, s_{*}, v, j, v\right)$ is called holomorphic if the map $v$ is holomorphic, i.e. if

$$
\bar{\partial}_{j, J}(v):=\frac{1}{2}(d v+J(v) d v \circ j)=0 .
$$

A stable map is a holomorphic configuration whose automorphism group is finite. This means that each genus- 0 component of $\Sigma$ on which $v$ is constant carries at least three special points and each genus- 1 component of $\Sigma$ on which $v$ is constant carries at least one special point. A component on which $v$ is constant is commonly called a ghost component so a stable map is a holomorphic configuration such that each ghost component is stable in the sense of [4, Definition 3.7]. The stable maps of type $(\mathrm{g}, \mathrm{n})$ are a subgroupoid of $\mathcal{B}_{\mathrm{g}, \mathrm{n}}(M, J)$; the orbit space $\overline{\mathcal{M}}_{\mathrm{g}, \mathrm{n}}$ of this subgroupoid is (set theoretically) the moduli space of stable maps of type $(\mathrm{g}, \mathrm{n})$. Similarly define the subset $\overline{\mathcal{M}}_{\mathrm{g}, \mathrm{n}, \mathrm{d}}$. Our goal is to construct a canonical orbifold structure on the regular part of this space.

Definition 2.3 A holomorphic configuration $\left(\Sigma, s_{*}, v, j, v\right)$ is called regular if

$$
\Omega_{j}^{0,1}\left(\Sigma, v^{*} T M\right)=\operatorname{im} D_{v}+d v \cdot \Omega_{j}^{0,1}(\Sigma, T \Sigma)
$$

where

$$
D_{v}: \Omega^{0}\left(\Sigma / \nu, v^{*} T M\right) \rightarrow \Omega_{j}^{0,1}\left(\Sigma, v^{*} T M\right)
$$

is the linearized Cauchy Riemann operator (see [2, p. 41] and 2.6 below).

2.4 Fix $v$ and $s_{*}$. Let $\mathcal{J}(\Sigma) \subset \operatorname{End}(T \Sigma)$ denote the manifold of complex structures on $\Sigma$ and let

$$
\mathcal{B}:=\mathcal{J}(\Sigma) \times C^{\infty}(\Sigma / v, M)
$$

Form the vector bundle $\mathcal{E} \rightarrow \mathcal{B}$ with fiber

$$
\mathcal{E}_{j, v}:=\Omega_{j}^{0,1}\left(\Sigma, v^{*} T M\right)
$$

and let $\mathcal{S}: \mathcal{B} \rightarrow \mathcal{E}$ denote the section defined by the nonlinear Cauchy-Riemann operator

$$
\mathcal{S}(j, v):=\bar{\partial}_{j, J}(v)
$$

A configuration $(j, v)$ is holomorphic and only if $\mathcal{S}(j, v)=0$. The intrinsic derivative of $\mathcal{S}$ at a zero $(j, v) \in \mathcal{S}^{-1}(0)$ is the operator $\mathcal{D}_{j, v}: T_{j, v} \mathcal{B} \rightarrow \mathcal{E}_{j, v}$ given by

$$
\mathcal{D}_{j, v}(\hat{\jmath}, \hat{v})=D_{v} \hat{v}+\frac{1}{2} J(v) d v \cdot \hat{\jmath} .
$$


A holomorphic configuration $(j, v)$ is regular if and only if the operator $\mathcal{D}_{j, v}$ is surjective. This follows from the following three assertions: (1) the tangent space to $\mathcal{B}$ at $(j, v)$ is

$$
T_{j, v} \mathcal{B}=\Omega_{j}^{0,1}(\Sigma, T \Sigma) \times \Omega^{0}\left(\Sigma, v^{*} T M\right)
$$

(2) When $v$ is holomorphic, we have $J(v) d v \cdot \hat{\jmath}=d v \cdot j \hat{\jmath}$. (3) The map

$$
\Omega_{j}^{0,1}(\Sigma, T \Sigma) \rightarrow \Omega_{j}^{0,1}(\Sigma, T \Sigma): \hat{\jmath} \mapsto j \hat{\jmath}
$$

is bijective. Hence, for a regular holomorphic configuration, the zero set of $\mathcal{S}$ is a Fréchet manifold near $(j, v)$ with tangent space $\operatorname{ker} \mathcal{D}_{j, v}$. This zero set is the "stratum" consisting of the holomorphic configurations of type $(\mathrm{g}, \mathrm{n})$ obtained by fixing $v$ and varying $(j, v)$. Fixing $j$ gives the vector bundle over $C^{\infty}(\Sigma / v, M)$ with fibers $\Omega_{j}^{0,1}\left(\Sigma, v^{*} T M\right)$. When the configuration $(j, v)$ is holomorphic, the operator $D_{v}$ is the intrinsic derivative of the section $v \mapsto \mathcal{S}(j, v)$.

2.5 The section $(j, v) \mapsto \mathcal{S}(j, v)=\bar{\partial}_{j, J}(v)$ is equivariant under the action of the group $\operatorname{Diff}(\Sigma, v)$ of orientation preserving diffeomorphisms that preserve the nodal structure. The Lie algebra of $\operatorname{Diff}(\Sigma, v)$ is the space

$$
\operatorname{Vect}(\Sigma, \nu):=\left\{\xi \in \Omega^{0}(\Sigma, T \Sigma) \mid \xi(z)=0 \forall z \in \cup \nu\right\}
$$

of vector fields on $\Sigma$ that vanish on the nodal set. The infinitesimal equivariance condition is

$$
D_{v}(d v \cdot \xi)=d v \cdot \bar{\partial}_{j} \xi
$$

for every $\xi \in \operatorname{Vect}(\Sigma, v)$. The diffeomorphism group $\operatorname{Diff}(\Sigma, v)$ acts on the space

$$
\mathcal{Z}_{\mathrm{n}}(\Sigma, v ; M, J):=\left(\Sigma^{\mathrm{n}} \backslash \Delta\right) \times \mathcal{S}^{-1}(0)
$$

(where $\Delta$ is the fat diagonal) by

$$
g^{*}\left(s_{1}, \ldots, s_{\mathrm{n}}, j, v\right):=\left(g^{-1}\left(s_{1}\right), \ldots, g^{-1}\left(s_{\mathrm{n}}\right), g^{*} j, v \circ g\right)
$$

for $g \in \operatorname{Diff}(\Sigma, v)$. Let $\mathcal{P}_{\mathrm{n}}(\Sigma, v ; M, J) \subset \mathcal{Z}_{\mathrm{n}}(\Sigma, v ; M, J)$ denote the subset of stable maps, i.e. the subset where $\operatorname{Diff}(\Sigma, v)$ acts with finite isotropy. Then the quotient space

$$
\mathcal{M}_{\mathrm{n}}(\Sigma, v ; M, J):=\mathcal{P}_{\mathrm{n}}(\Sigma, v ; M, J) / \operatorname{Diff}(\Sigma, v)
$$

is a stratum of the moduli space $\overline{\mathcal{M}}_{\mathrm{g}, \mathrm{n}}(M, J)$ of all stable maps of genus $\mathrm{g}$ with $\mathrm{n}$ marked points. The stratum can also be expressed as the quotient $\mathcal{M}_{\mathrm{n}}(\Sigma, v ; M, J)=\mathcal{S}^{-1}(0)_{\text {stable }}$ / $\operatorname{Diff}\left(\Sigma, v, s_{*}\right)$ where $\operatorname{Diff}\left(\Sigma, v, s_{*}\right) \subset \operatorname{Diff}(\Sigma, v)$ denotes the subgroup of all diffeomorphisms $\phi \in \operatorname{Diff}(\Sigma, v)$ that satisfy $\phi\left(s_{\mathrm{i}}\right)=s_{\mathrm{i}}$ for $\mathrm{i}=1, \ldots, \mathrm{n}$.

2.6 Let $(\Sigma, v, j)$ be a nodal Riemann surface and $v: \Sigma \rightarrow M$ be a smooth map. Fix a connection on $T M$ and define

$$
D_{v} \hat{v}:=\frac{1}{2}(\nabla \hat{v}+J(v) \nabla \hat{v} \circ j)-\frac{1}{2} J(v) \nabla_{\hat{v}} J(v) \partial_{j, J}(v) .
$$

(See [2, p. 41].) The definition for $D_{v}$ is meaningful even when $J$ is not integrable. If $\bar{\partial}_{j, J}(v)=0$, then the right hand side of (4) is independent of the choice of the connection $\nabla$ and is the operator of Definition 2.3. If $J$ is integrable, $v^{*} T M \rightarrow \Sigma$ is a holomorphic vector bundle and $D_{v}$ is its Cauchy Riemann operator. If $\nabla$ is the Levi Civita connection of a Kähler metric, then $\nabla J=0$ and the last term vanishes. In general (assuming neither integrability nor that $(j, v)$ is a zero) the formula for $D_{v}$ still defines a Cauchy-Riemann operator on $v^{*} T M$ which depends however on the connection and might not be complex linear, but it is always Fredholm. 


\section{Unfoldings of stable maps}

3.1 Fix two nonnegative integers $\mathrm{g}$ and $\mathrm{n}$ and a homology class $\mathrm{d} \in H_{2}(M ; \mathbb{Z}$ ). A (holomorphic) family of maps (of type $(g, n, d)$ ) is a triple

$$
\left(\pi: Q \rightarrow B, S_{*}, H\right)
$$

where $\left(\pi, S_{*}\right)$ is a marked nodal Riemann family (of type $\left.(\mathrm{g}, \mathrm{n})\right)$ and

$$
H: Q \rightarrow M
$$

is a holomorphic map such that the restriction of $H$ to each fiber $Q_{b}$ represents the homology class d. A desingularization $u: \Sigma \rightarrow Q_{b}$ of a fiber induces a holomorphic configuration $\left(\Sigma, s_{*}, v, j, v\right)$ with

$$
v:=H \circ u \text {. }
$$

The family of maps is called stable if each configuration that arises from a desingularization of a fiber is a stable map. Given two families of maps $\left(\pi_{A}: P \rightarrow A, R_{*}, H_{A}\right)$ and $\left(\pi_{B}: Q \rightarrow B, S_{*}, H_{B}\right)$ a map $f: P_{a} \rightarrow Q_{b}$ is called a fiber isomorphism if it is a fiber isomorphism of marked nodal Riemann families and

$$
H_{A} \mid P_{a}=H_{B} \circ f \text {. }
$$

A morphism between two families of maps $\left(\pi_{A}, R_{*}, H_{A}\right)$ and $\left(\pi_{B}, S_{*}, H_{B}\right)$ is a commutative diagram

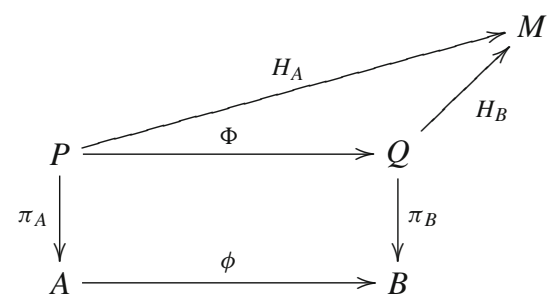

such that, for each $a \in A$, the restriction of $\Phi$ to the fiber $P_{a}$ is a fiber isomorphism. The morphism is called continuous, continuously differentiable, smooth, or holomorphic if both maps $\phi$ and $\Phi$ are.

Definition 3.2 An unfolding of maps is a quadruple $\left(\pi_{B}, S_{*}, H_{B}, b\right)$ where $\left(\pi_{B}, S_{*}, H_{B}\right)$ is a family of maps and $b \in B$. An unfolding $\left(\pi_{B}, S_{*}, H_{B}, b\right)$ is called universal if, for every other unfolding $\left(\pi_{A}, R_{*}, H_{A}, a\right)$ and every fiber isomorphism $f: P_{a} \rightarrow Q_{b}$, there is a unique morphism

$$
(\phi, \Phi):\left(\pi_{A}, R_{*}, H_{A}, a\right) \rightarrow\left(\pi_{B}, S_{*}, H_{B}, b\right)
$$

of families of maps such that

$$
\Phi \mid P_{a}=f
$$

This is to be understood in the sense of germs; the morphism may only be defined after shrinking $A$, and two morphisms are considered equal if they agree on some neighborhood of $P_{a}$. 
Definition 3.3 Let $\left(\pi: Q \rightarrow B, S_{*}, H, b\right)$ be an unfolding of maps and $u: \Sigma \rightarrow Q_{b}$ be a desingularization with induced structures $s_{*}, v, j$, and $v$ on $\Sigma$ Define the spaces

$$
\begin{aligned}
\mathcal{X}_{u} & :=\left\{\hat{u} \in \Omega^{0}\left(\Sigma / \nu, u^{*} T Q\right) \mid d \pi(u) \hat{u} \equiv \text { constant, } \hat{u}\left(s_{\mathrm{i}}\right) \in T_{u\left(s_{\mathrm{i}}\right)} S_{\mathrm{i}}\right\}, \\
\mathcal{Y}_{u} & :=\left\{\eta \in \Omega_{j}^{0,1}\left(\Sigma, u^{*} T Q\right) \mid d \pi(u) \eta=0\right\}, \\
\mathcal{X}_{v} & :=\Omega^{0}\left(\Sigma / \nu, v^{*} T M\right), \quad \mathcal{Y}_{v}:=\Omega_{j}^{0,1}\left(\Sigma, v^{*} T M\right) .
\end{aligned}
$$

Consider the diagram

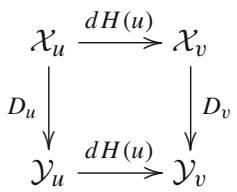

where the vertical maps are the restrictions to the indicated subspaces of the linearized Cauchy-Riemann operators (see 2.6)

$$
\begin{aligned}
& D_{u}: \Omega^{0}\left(\Sigma, u^{*} T Q\right) \rightarrow \Omega^{0,1}\left(\Sigma, u^{*} T Q\right), \\
& D_{v}: \Omega^{0}\left(\Sigma, v^{*} T M\right) \rightarrow \Omega^{0,1}\left(\Sigma, v^{*} T M\right)
\end{aligned}
$$

associated to the holomorphic maps $u$ and $v$. Thus $D_{v}$ is the intrinsic derivative in 2.3. The diagram (5) commutes because $H$ is holomorphic and hence $\bar{\partial}_{j, J_{M}}(H \circ u)=d H(u) \cdot \bar{\partial}_{j, J_{Q}}(u)$. The commutative diagram (5) determines maps

$$
d H(u): \operatorname{ker} D_{u} \rightarrow \operatorname{ker} D_{v}, \quad d H(u): \operatorname{coker} D_{u} \rightarrow \operatorname{coker} D_{v}
$$

The unfolding is called infinitesimally universal if the maps in (6) are both bijective.

Remark 3.4 Let $\left(\Sigma, s_{*}, v, j, v\right)$ be induced by a desingularization $u: \Sigma \rightarrow Q_{b}$ of an unfolding $\left(\pi: Q \rightarrow B, S_{*}, H, b\right)$. Then $\left(\Sigma, s_{*}, v, j, v\right)$ is regular if and only if the map $d H(u)$ : $\operatorname{coker} D_{u} \rightarrow \operatorname{coker} D_{v}$ is surjective. To see this note that $d H(u): \operatorname{coker} D_{u} \rightarrow \operatorname{coker} D_{v}$ is surjective if and only if

$$
\mathcal{Y}_{v}=\operatorname{im} D_{v}+\operatorname{im}\left(d H(u): \mathcal{Y}_{u} \rightarrow \mathcal{Y}_{v}\right)
$$

Since $u$ is an immersion, the map

$$
T_{j} \mathcal{J}(\Sigma)=\Omega_{j}^{0,1}(\Sigma, T \Sigma) \rightarrow \mathcal{Y}_{u}: \eta \mapsto d u \cdot \eta
$$

is an isomorphism. But $v=H \circ u$ so $d v \cdot \eta=d H(u) \circ d u \cdot \eta$ so

$$
d v \cdot \Omega_{j}^{0,1}(\Sigma, T \Sigma)=\operatorname{im}\left(d H(u): \mathcal{Y}_{u} \rightarrow \mathcal{Y}_{v}\right) .
$$

Hence equation (1) is equivalent to equation (7) which asserts that the holomorphic configuration $\left(\Sigma, s_{*}, v, j, v\right)$ is regular.

When $M$ is a point the above definitions and the following theorems agree with the corresponding ones in [4].

Theorem 3.5 A holomorphic configuration $\left(\Sigma, s_{*}, v, j, v\right)$ admits an infinitesimally universal unfolding if and only if it is a regular stable map. 
Proof The hard part of the proof is to show that 'if' holds under the additional assumption that the underlying marked nodal Riemann surface $\left(\Sigma, s_{*}, v, j\right)$ is stable. We will prove this in Sect. 7. Here we give the easy parts of the proof.

We prove 'if' (assuming the aforementioned result of Sect. 7). By adding marked points in the appropriate components we may construct a stable map whose underlying marked nodal Riemann surface is stable. Hence, by backwards induction, it is enough to prove the following

Claim If a stable map admits an infinitesimally universal unfolding and the configuration which results on deleting a marked point is also a stable map, then it too admits an infinitesimally universal unfolding.

To prove the claim let $\left(\pi: Q \rightarrow B, S_{1}, \ldots, S_{\mathrm{n}}, H, b_{0}\right)$ be an infinitesimally universal unfolding of $\left(\Sigma, s_{1}, \ldots, s_{\mathrm{n}}, v, j, v\right)$ with associated desingularization $u: \Sigma \rightarrow Q_{b_{0}}$ and assume that $\left(\Sigma, s_{1}, \ldots, s_{\mathrm{n}-1}, v, j, v\right)$ is still stable. We will construct an infinitesimally universal unfolding $\left(\pi: Q^{\prime} \rightarrow B^{\prime}, S_{1}^{\prime}, \ldots, S_{\mathrm{n}-1}^{\prime}, H^{\prime}, b_{0}\right)$ such that $B^{\prime}$ is a submanifold of $B, Q^{\prime}:=\pi^{-1}\left(B^{\prime}\right)$ is a submanifold of $Q, H^{\prime}:=H \mid Q^{\prime}$, and $S_{\mathrm{i}}^{\prime}=S_{\mathrm{i}} \cap Q^{\prime}$ for $\mathrm{i}=1, \ldots, \mathrm{n}-1$. Define the space

$$
\widetilde{\mathcal{X}}_{u}:=\left\{\hat{u} \in \Omega^{0}\left(\Sigma / v, u^{*} T Q\right) \mid d \pi(u) \hat{u} \equiv \text { constant, } \hat{u}\left(s_{\mathrm{i}}\right) \in T_{u\left(s_{\mathrm{i}}\right)} S_{\mathrm{i}} \text { for } \mathrm{i}<\mathrm{n}\right\} .
$$

Note that $\widetilde{\mathcal{X}}_{u}$ is obtained from $\mathcal{X}_{u}$ by removing the constraint on the value $\hat{u}\left(s_{\mathrm{n}}\right)$ at the last marked point. Thus $\mathcal{X}_{u}$ is a subspace of $\widetilde{\mathcal{X}}_{u}$ of complex codimension one; a complement of $\mathcal{X}_{u}$ in $\widetilde{\mathcal{X}}_{u}$ is spanned by any vertical vector field along $u$, satisfying the nodal condition, that vanishes at the marked points $s_{\mathrm{i}}$ for $\mathrm{i}<1$ and does not vanish at $s_{\mathrm{n}}$. Denote by

$$
\widetilde{D}_{u}: \widetilde{\mathcal{X}}_{u} \rightarrow \mathcal{Y}_{u}
$$

the operator given by the same formula as $D_{u}$ on the larger domain. Note that the diagram (5) continues to commute when we replace $\mathcal{X}_{u}$ and $D_{u}$ by $\widetilde{\mathcal{X}}_{u}$ and $\widetilde{D}_{u}$, respectively. We prove the following.

(a) $\operatorname{im} D_{u}=\operatorname{im} \widetilde{D}_{u}$ and $\operatorname{ker} D_{u} \subset \operatorname{ker} \widetilde{D}_{u}$ is a subspace of codimension one.

(b) There is an element $\hat{u} \in \operatorname{ker} \widetilde{D}_{u}$ with $d H(u) \hat{u} \equiv 0$ and $\hat{b}:=d \pi(u) \hat{u} \neq 0$.

With this understood we choose a complex submanifold $B^{\prime} \subset B$ of codimension one such that $\pi$ is tranverse to $B^{\prime}$ and $\hat{b} \notin T_{b_{0}} B^{\prime}$. Then the kernel of the resulting operator $D_{u}^{\prime}$ is a complex subspace of the kernel of $\widetilde{D}_{u}$ of codimension one. Since $\hat{b} \notin T_{b_{0}} B^{\prime}$, the kernel of $D_{u}^{\prime}$ is mapped under $d H(u)$ isomorphically onto the kernel of $D_{v}$. Since $D_{u}^{\prime}$ has the same image as $\widetilde{D}_{u}$ and $D_{u}$ we deduce that $d H(u)$ also induces an isomorphism from the cokernel of $D_{u}^{\prime}$ to that of $D_{v}$. Hence $\left(\pi: Q^{\prime} \rightarrow B^{\prime}, S_{1}^{\prime}, \ldots, S_{\mathrm{n}-1}^{\prime}, H^{\prime}, b_{0}\right)$ is an infinitesimally universal unfolding of $\left(\Sigma, s_{1}, \ldots, s_{\mathrm{n}-1}, v, j, v\right)$ as claimed.

It remains to prove (a) and (b). To prove (a) note that $\widetilde{D}_{u}$ has the same image as $D_{u}$. (If $\eta \in \mathcal{Y}_{u}$ belongs to the image of $\widetilde{D}_{u}$ then $d H(u) \eta \in \operatorname{im} D_{v}$ and, since the second map in (6) is injective, this implies that $\eta$ belongs to the image of $D_{u}$.) Hence (a) follows from the fact that $\mathcal{X}_{u}$ has codimension one in $\widetilde{\mathcal{X}}_{u}$. To prove (b) we use the fact that the first map in (6) is surjective and $d H(u)$ maps the kernel of $\widetilde{D}_{u}$ to the kernel of $D_{v}$. Hence there is an element

$$
\hat{u} \in \operatorname{ker} \widetilde{D}_{u} \cap \operatorname{ker} d H(u) \backslash \operatorname{ker} D_{u} .
$$

Any such element satisfies

$$
d \pi(u) \hat{u} \neq 0 .
$$


Otherwise there is a vector field $\xi \in \operatorname{Vect}(\Sigma)$ with $\hat{u}=d u \cdot \xi$; since $\hat{u} \in \widetilde{\mathcal{X}}_{u}$ this implies that $\xi$ belongs to the Lie algebra of the stabilizer subgroup of $\left(\Sigma, s_{1}, \ldots, s_{\mathrm{n}-1}, v, j, v\right)$, contradicting stablility. Thus we have proved (a) and (b) and hence the claim.

We prove 'only if'. Let $\left(\Sigma, s_{*}, v, j, v\right)$ be induced by a desingularization $u: \Sigma \rightarrow Q_{b}$ of the infinitesimally universal unfolding $\left(\pi: Q \rightarrow B, S_{*}, H, b\right)$. Then the holomorphic configuration $\left(\Sigma, s_{*}, v, j, v\right)$ is regular, by Remark 3.4. Next we argue as in [4]. Assume that $\left(\Sigma, s_{*}, v, j, v\right)$ is regular but not stable. Then either $\Sigma$ has genus one, $v$ is constant, and there are no special points or else $\Sigma$ contains a component of genus zero on which $v$ is constant and which carries at most two special points. In either case there is an abelian complex Lie group $A$ (namely $A=\Sigma$ in the former case and $A=\mathbb{C}^{*}$ in the latter) and an effective holomorphic action

$$
A \times \Sigma \rightarrow \Sigma:(a, z) \mapsto a_{\Sigma}(z)
$$

that preserves the given structures. Let $P:=A \times \Sigma, \pi_{A}$ be the projection on the first factor, $R_{*}:=A \times s_{*}, f_{A}(a, z):=v(z)$, and $a_{0} \in A$ be the identity. If $u_{0}: \Sigma \rightarrow Q$ is any desingularization of a fiber $Q_{b_{0}}$ of an unfolding ( $\left.\pi_{B}: Q \rightarrow B, S_{*}, f_{B}, b_{0}\right)$ which induces the given structures on $\Sigma$, then

$$
\Phi_{1}(a, z):=u_{0}(z), \quad \Phi_{2}(a, z):=u_{0}\left(a_{\Sigma}(z)\right)
$$

are distinct morphisms from $\left(\pi_{A}, R_{*}, f_{A}, a_{0}\right)$ to $\left(\pi_{B}, S_{*}, f_{B}, b_{0}\right)$ which extend the fiber isomorphism $P_{a_{0}} \rightarrow Q_{b_{0}}:\left(a_{0}, z\right) \mapsto u_{0}(z)$. Hence $\left(\pi_{B}, S_{*}, f_{B}, b_{0}\right)$ is not a universal unfolding.

Theorem 3.6 An unfolding of a regular stable map is universal if and only if it is infinitesimally universal.

Proof We prove 'if' in Sect. 7. For 'only if' we argue as in [4]. A composition of morphisms (of nodal families of maps) is again a morphism. The only morphism which is the identity on the central fiber of a universal unfolding is the identity. It follows that any two universal unfoldings of the same holomorphic configuration are isomorphic. By Theorem 3.5 there is an infinitesimally universal unfolding and by 'if' it is universal and hence isomorphic to every other universal unfolding. Any unfolding isomorphic to an infinitesimally universal unfolding is itself infinitesimally universal.

Example 3.7 Here is an example of an unfolding which is universal but not infinitesimally universal. Let $B=\mathbb{C}, b_{0}=0, \Sigma$ be a Riemann surface of genus $\mathrm{g} \geq 1, Q=M=B \times \Sigma$, $\pi_{B}: Q \rightarrow B$ be the projection on the first factor, and $H_{B}: Q \rightarrow M$ be the identity map. This is trivially universal as follows. If $\left(\pi_{A}, H_{A}, a_{0}\right)$ is another unfolding and $f_{0}: P_{a_{0}} \rightarrow Q_{b_{0}}$ is a fiber isomorphism as in 3.1, then $f_{0}=H_{A} \mid P_{a_{0}}$, the unique solution of $H_{B} \circ \Phi=H_{A}$ is $\Phi=H_{A}$, and $\phi$ is uniquely determined by the condition $\pi_{B} \circ \Phi=\phi \circ \pi_{A}$. To show that that the example is not infinitesimally universal it is enough (by Theorem 3.5) to show that the fiber is not regular, i.e. that

$$
\operatorname{im} D_{v}+d v \cdot \Omega_{j}^{0,1}(\Sigma, T \Sigma) \subsetneq \Omega_{j}^{0,1}(\Sigma, T M)
$$

where $v: \Sigma \rightarrow M$ is the map $v(z):=\left(b_{0}, z\right)$. Now $T M$ is the direct sum of $d v \cdot T \Sigma$ with a trivial bundle, so it is enough to show that $D_{v}$ followed by projection of the trivial bundle is not surjective. But this is the linear operator $\bar{\partial}: \Omega^{0}(\Sigma) \rightarrow \Omega_{j}^{0,1}(\Sigma)$. Its cokernel is the space of holomorphic 1-forms and it has dimension $\mathrm{g}$.

Theorem 3.8 If an unfolding $\left(\pi, S_{*}, H, b_{0}\right)$ is infinitesimally universal, then the unfolding $\left(\pi, S_{*}, H, b\right)$ is infinitesimally universal for $b$ sufficiently near $b_{0}$. 
3.9 Fix two nonnegative integers $\mathrm{g}$ and $\mathrm{n}$ and a homology class $\mathrm{d} \in H_{2}(M ; \mathbb{Z})$. A universal family of maps of type $(\mathrm{g}, \mathrm{n}, \mathrm{d})$ is a marked nodal family of maps $\left(\pi_{B}: Q \rightarrow B, S_{*}, H_{B}\right)$ satisfying the following conditions.

(1) $\left(\pi_{B}, S_{*}, H_{B}, b\right)$ is a universal unfolding of maps of type ( $\left.\mathrm{g}, \mathrm{n}, \mathrm{d}\right)$ for every $b \in B$.

(2) Every regular stable map of type $(g, n, d)$ arises from a desingularization of at least one fiber of $\pi_{B}$.

(3) $B$ is second countable.

The existence of a universal marked nodal family of maps for every triple $(\mathrm{g}, \mathrm{n}, \mathrm{d})$ follows immediately from Theorems 3.5, 3.6, and 3.8 as in [4, Proposition 6.3].

3.10 Every universal family $\left(\pi_{B}: Q \rightarrow B, S_{*}, H_{B}\right.$ ) of maps of type ( $\left.\mathrm{g}, \mathrm{n}, \mathrm{d}\right)$ determines a groupoid $(B, \Gamma, s, t, e, i, m)$ as in [4, Definition 6.4]; here $\Gamma$ denotes the set of all triples $(a, f, b)$ such that $a, b \in B$ and $f: Q_{a} \rightarrow Q_{b}$ is a fiber isomorphism satisfying $H_{B} \circ f=H_{B} \mid Q_{a}$, and the structure maps $s, t: \Gamma \rightarrow B, e: B \rightarrow \Gamma, i: \Gamma \rightarrow \Gamma$, and $m: \Gamma_{s} \times_{t} \Gamma \rightarrow \Gamma$ are defined by

$$
\begin{aligned}
s(a, f, b) & :=a, \quad t(a, f, b):=b, \quad e(a):=(a, \mathrm{id}, a), \\
i(a, f, b) & :=\left(b, f^{-1}, a\right), \quad m((b, g, c),(a, f, b)):=(a, g \circ f, c) .
\end{aligned}
$$

The associated groupoid is equipped with a functor $B \rightarrow \overline{\mathcal{B}}_{\mathrm{g}, \mathrm{n}, \mathrm{d}}^{\text {reg }}(M, J): b \mapsto \Sigma_{b}$ to the groupoid of Definition 2.3, i.e. $\iota_{b}: \Sigma_{b} \rightarrow Q_{b}$ denotes the canonical desingularization in [4, Remark 4.4]. By definition the induced map

$$
B / \Gamma \rightarrow \overline{\mathcal{M}}_{\mathrm{g}, \mathrm{n}, \mathrm{d}}^{\mathrm{reg}}(M, J)
$$

on orbit spaces is bijective. As in [4, Theorem 6.5] the groupoid $(B, \Gamma)$ equips the moduli space $\overline{\mathcal{M}}_{\mathrm{g}, \mathrm{n}, \mathrm{d}}^{\text {reg }}(M, J)$ with an orbifold structure which is independent of the choice of the universal family.

Theorem 3.11 Let $\left(\pi_{B}: Q \rightarrow B, S_{*}, H_{B}\right)$ be a universal family of maps of type $(\mathrm{g}, \mathrm{n}, \mathrm{d})$ as in 3.9. Then the associated groupoid $(B, \Gamma)$ constructed in 3.10 is proper in the sense of $[4,2.2]$.

Proof See Sect. 7.

Corollary 3.12 Fix a homology class $\mathrm{d} \in \mathrm{H}_{2}(M ; \mathbb{Z})$. Then the moduli space $\overline{\mathcal{M}}_{\mathrm{g}, \mathrm{n}, \mathrm{d}}^{\mathrm{reg}}(M, J)$ of isomorphism classes of regular stable maps of genus $\mathrm{g}$ with $\mathrm{n}$ marked points representing the class $\mathrm{d}$ is a complex orbifold of dimension

$$
\operatorname{dim}_{\mathbb{C}} \overline{\mathcal{M}}_{\mathrm{g}, \mathrm{n}, \mathrm{d}}^{\mathrm{reg}}(M, J)=(g-1)\left(3-\operatorname{dim}_{\mathbb{C}} M\right)+\left\langle c_{1}(T M), \mathrm{d}\right\rangle+\mathrm{n} .
$$

Remark 3.13 If $(M, \omega, J)$ is a Kähler manifold with a transitive action by a compact Lie group $G$, then every genus zero configuration in $M$ is regular (see [5] or [2, Proposition 7.4.3]). Hence the moduli space $\overline{\mathcal{M}}_{0, \mathrm{n}, \mathrm{d}}(M, J)$ is a (compact) complex orbifold for every $\mathrm{d} \in H_{2}(M ; \mathbb{Z})$. For $M=\mathbb{C} P^{\mathrm{m}}$ this result is due to Fulton and Pandharipande [1]. Their result applies to all projective manifolds whenever all the stable maps are regular. In such cases they show that the moduli space is an algebraic orbifold. In contrast, our result shows that the set of regular maps into any complex manifold is an orbifold. 


\section{Stable maps without nodes}

In this section we restrict attention to regular stable maps without nodes. Let $\left(\Sigma, s_{*}, j_{0}, v_{0}\right)$ be a regular stable map of type $(\mathrm{g}, \mathrm{n}, \mathrm{d})$ without nodes. We will construct an infinitesimally universal unfolding $\left(\pi_{B}, S_{*}, H_{B}, b_{0}\right)$ of $\left(\Sigma, s_{*}, j_{0}, v_{0}\right)$, show that it is universal, and prove that every other infinitesimally universal unfolding of $\left(\Sigma, s_{*}, j_{0}, v_{0}\right)$ is isomorphic to the one we've constructed.

4.1 Fix two nonnegative integers $\mathrm{n}$ and $\mathrm{g}$, a homology class $\mathrm{d} \in \mathrm{H}_{2}(M ; \mathbb{Z})$, and a compact oriented surface $\Sigma$ without boundary of genus $g$. Denote

$$
\mathcal{P}:=\left\{\begin{array}{l|l}
\left(s_{1}, \ldots, s_{\mathrm{n}}, j, v\right) & \begin{array}{l}
s_{*} \in \Sigma^{\mathrm{n}} \backslash \Delta, j \in \mathcal{J}(\Sigma), v \in C^{\infty}(\Sigma, M) \\
\bar{\partial}_{j, J}(v)=0,[v]=\mathrm{d} \\
\mathcal{D}_{j, v} \text { is onto, }\left(s_{*}, j, v\right) \text { is stable }
\end{array}
\end{array}\right\}
$$

where $\Delta \subset \Sigma^{\mathrm{n}}$ denotes the fat diagonal, $[v]:=v_{*}[\Sigma]$ denotes the homology class represented by $v$, and

$$
\mathcal{D}_{j, v}: \Omega_{j}^{0,1}(\Sigma, T \Sigma) \times \Omega^{0}\left(\Sigma, v^{*} T M\right) \rightarrow \Omega^{0,1}\left(\Sigma, v^{*} T M\right)
$$

denotes the linearized Cauchy-Riemann operator of 2.4. Thus $\mathcal{P}$ is the regular part of the space $\mathcal{P}_{\mathrm{n}, \mathrm{d}}(\Sigma ; M, J)$ in 2.5. The group

$$
\mathcal{G}:=\operatorname{Diff}_{0}(\Sigma)
$$

of orientation preserving diffeomorphisms of $\Sigma$ that are isotopic to the identity acts on $\mathcal{P}$ as in equation (3):

$$
g^{*}\left(s_{1}, \ldots, s_{\mathrm{n}}, j, v\right):=\left(g^{-1}\left(s_{1}\right), \ldots, g^{-1}\left(s_{\mathrm{n}}\right), g^{*} j, g^{*} v\right)
$$

for $g \in \mathcal{G}$.

Remark 4.2 Roughly speaking, the tuple $\left(\mathcal{Q} \rightarrow \mathcal{B}, \mathcal{S}_{*}, \mathcal{H}\right)$ defined by

$$
\begin{aligned}
\mathcal{B} & :=\mathcal{P} / \mathcal{G}, \quad \mathcal{Q}:=\mathcal{P} \times \mathcal{G} \Sigma \\
\mathcal{H}\left(\left[s_{1}, \ldots, s_{\mathrm{n}}, j, v, z\right]\right) & :=v(z), \quad \mathcal{S}_{\mathrm{i}}:=\left\{\left[s_{1}, \ldots, s_{\mathrm{n}}, j, v, z\right] \in \mathcal{Q} \mid z=s_{\mathrm{i}}\right\}
\end{aligned}
$$

is a universal family. Our task is to make sense of these quotients. In the case

$$
\mathrm{n}>2-2 \mathrm{~g}
$$

the action is free. In general, the action is only semi-free, i.e. the isotropy group of a point in $\mathcal{P}$ is always finite but it might be nontrivial. (Example: $\mathrm{n}=0, \Sigma=M=S^{2}, v(z)=z^{2}$.) In this case the quotient spaces $\mathcal{B}$ and $\mathcal{Q}$ cannot be manifolds and hence do not qualify as universal unfoldings. However, we shall prove that even in this case every point in $\mathcal{P}$ admits a holomorphic local slice for the $\mathcal{G}$-action and that these slices can be used to construct universal unfoldings.

4.3 The space $\mathcal{P}$ is an infinite dimensional Frechét manifold. Its tangent space at a point $p=\left(s_{*}, j, v\right) \in \mathcal{P}$ is the space $T_{p} \mathcal{P}$ of all tuples $\hat{p}=\left(\hat{s}_{*}, \hat{\jmath}, \hat{v}\right)$ with $\hat{s}_{\mathrm{i}} \in T_{s_{\mathrm{i}}} \Sigma, \hat{\jmath} \in T_{j} \mathcal{J}(\Sigma)$, $\hat{v} \in \Omega^{0}\left(\Sigma, v^{*} T M\right)$ that satisfy

$$
D_{v} \hat{v}+\frac{1}{2} J(v) d v \circ \hat{\jmath}=0 .
$$


The Lie algebra of $\mathcal{G}$ is $\operatorname{Lie}(\mathcal{G})=\operatorname{Vect}(\Sigma)$ and its (contravariant) infinitesimal action at $p \in \mathcal{P}$ is the operator $\mathcal{L}_{p}: \operatorname{Vect}(\Sigma) \rightarrow T_{p} \mathcal{P}$ defined by

$$
\mathcal{L}_{p} \xi:=\left.\frac{d}{d t} g_{t}^{*} p\right|_{t=0}
$$

where $p=\left(s_{*}, j, v\right) \in \mathcal{P}$ and $\mathbb{R} \rightarrow \mathcal{G}: t \mapsto g_{t}$ satisfies

$$
g_{0}=\mathrm{id},\left.\quad \frac{d}{d t} g_{t}\right|_{t=0}=\xi .
$$

(The right hand side of (9) is independent of the choice of $g_{t}$ satisfying (10).) Since $2 j \bar{\partial}_{j} \xi=$ $\mathcal{L}_{\xi} j \in T_{j} \mathcal{J}(\Sigma)$ is the Lie derivative of $j$ in the direction $\xi$, equation (9) may be written

$$
\mathcal{L}_{p} \xi=\left(-\xi\left(s_{1}\right), \ldots,-\xi\left(s_{\mathrm{n}}\right), 2 j \bar{\partial}_{j} \xi, d v \cdot \xi\right), \quad p=\left(s_{1}, \ldots, s_{\mathrm{n}}, j, v\right) .
$$

The image of $\mathcal{L}_{p}$ is the tangent space $T_{p} \mathcal{G}^{*} p$ to the $\mathcal{G}$-orbit of $p$. The space $T_{p} \mathcal{P}$ carries a natural complex structure $\mathcal{I}(p): T_{p} \mathcal{P} \rightarrow T_{p} \mathcal{P}$ given by

$$
\mathcal{I}(p)\left(\hat{s}_{1}, \ldots, \hat{s}_{\mathrm{n}}, \hat{\jmath}, \hat{v}\right):=\left(j\left(s_{1}\right) \hat{s}_{1}, \ldots, j\left(s_{\mathrm{n}}\right) \hat{s}_{\mathrm{n}}, j \hat{\jmath}, J(v) \hat{v}\right)
$$

for $p=\left(s_{1}, \ldots, s_{\mathrm{n}}, j, v\right) \in \mathcal{P}$. The tangent space $T_{p} \mathcal{P}$ is invariant under $\mathcal{I}(p)$ because the differential $d v$ and the operator $D_{v}$ are complex linear. The $\mathcal{G}$-action preserves this complex structure and the formula

$$
\mathcal{L}_{p} j \xi=\mathcal{I}(p) \mathcal{L}_{p} \xi, \quad p=\left(s_{*}, j, v\right) \in \mathcal{P},
$$

shows that $T_{p} \mathcal{G}^{*} p$ is a complex subspace of $T_{p} \mathcal{P}$. In other words, the orbits of $\mathcal{G}$ are complex submanifolds of $\mathcal{P}$ and the complex structure descends to the quotient $\mathcal{P} / \mathcal{G}$. The space $\mathcal{P}$ (without marked points) is the zero set of the section $(j, v) \mapsto \bar{\partial}_{j, J}(v)$ of an infinite dimensional vector bundle. The intrinsic differential of this section at a zero $(j, v)$ is the operator $\mathcal{D}_{j, v}$ in 2.4 and this operator is surjective by assumption. Condition (8) asserts that the pair $(\hat{\jmath}, \hat{v})$ belongs to the kernel of $\mathcal{D}_{j, v}$. Choosing a suitable Sobolev completion $\mathcal{P}^{s}$ of $\mathcal{P}$ (see the proof of Theorem 4.6 below) we can deduce that $\mathcal{P}^{s}$ is a smooth Hilbert manifold whose tangent space is given by (8). The action of $\mathcal{G}$ on this Hilbert manifold is not smooth; on any Sobolev completion its differential takes values in another Sobolev completion with one derivative less. However, in the Frechét category, where $B$ is a finite dimensional smooth manifold, the notion of a smooth map $\iota: B \rightarrow \mathcal{P}$ and its differential $d_{\iota}(b): T_{b} B \rightarrow T_{\iota(b)} \mathcal{P}$ have well defined meanings via evaluation maps.

Lemma 4.4 Let A be a complex manifold (with complex structure $\sqrt{-1}$ ),

$$
A \rightarrow \mathcal{P}: a \mapsto p(a)=\left(r_{1}(a), \ldots, r_{\mathrm{n}}(a), j(a), v(a)\right)
$$

be a smooth map and $\eta: T A \rightarrow \operatorname{Vect}(\Sigma)$ be a 1-form on A with values in the space of vector fields on $\Sigma$ such that

$$
\eta(a, \sqrt{-1} \hat{a})=-j(a) \eta(a, \hat{a})
$$

for all $(a, \hat{a}) \in T A$. Define an almost complex structure $J_{P}$ on $P:=A \times \Sigma$, sections $R_{1}, \ldots, R_{\mathrm{n}} \subset P$, and a map $H_{A}: P \rightarrow M$ by

$$
\begin{gathered}
J_{P}(a, z)(\hat{a}, \hat{z}):=(\sqrt{-1} \hat{a}, j(a)(z) \hat{z}+\eta(a, \hat{a})(z)), \\
R_{\mathrm{i}}:=\left\{\left(a, r_{\mathrm{i}}(a)\right) \mid a \in A\right\}, \quad H_{A}(a, z):=v(a)(z) .
\end{gathered}
$$

Then the following are equivalent. 
(i) The tuple $\left(\pi_{A}, R_{*}, H_{A}\right)$ is a (holomorphic) family of maps, i.e. $J_{P}$ is integrable, each $R_{\mathrm{i}}$ is a complex submanifold of $P$, and $H_{A}: P \rightarrow M$ is holomorphic.

(ii) $p$ and $\eta$ satisfy the differential equation

$$
d p(a) \hat{a}+\mathcal{I}(p(a)) d p(a) \sqrt{-1} \hat{a}-\mathcal{L}_{p(a)} \eta(a, \sqrt{-1} \hat{a})=0
$$

for every $a \in A$ and every $\hat{a} \in T_{a} A$.

Proof We prove that (i) implies (ii). If the almost complex structure $J_{P}$ is integrable then, by $[4$, Corrigendum, Lemma A], we have

$$
d j(a) \hat{a}+j(a) d j(a) \sqrt{-1} \hat{a}-\mathcal{L}_{\eta(a, \sqrt{-1} \hat{a})} j(a)=0 .
$$

Moreover, for $\mathrm{i}=1, \ldots, \mathrm{n}$ the set $R_{\mathrm{i}}$ is a complex submanifold of $A \times \Sigma$, if and only if

$$
d r_{\mathrm{i}}(a) \hat{a}+j(a) d r_{\mathrm{i}}(a) \sqrt{-1} \hat{a}+\eta(a, \sqrt{-1} \hat{a})\left(r_{\mathrm{i}}(a)\right)=0
$$

and $H_{A}: A \times \Sigma \rightarrow M$ is holomorphic if and only if

$$
(d v(a) \hat{a})(z)+J(v(a)(z))(d v(a) \sqrt{-1} \hat{a})(z)-d(v(a))(z) \eta(a, \sqrt{-1} \hat{a})(z)=0 .
$$

In the last formula $(d v(a) \hat{a})(z)$ denotes the derivative of $v(a)(z)$ with respect to $a$ and $d(v(a))(z) \hat{z}$ denotes the derivative of $v(a)(z)$ with respect to $z$.

This proves that (i) implies (ii). Conversely, assume (ii) and, without loss of generality, that $A$ is an open set in $\mathbb{C}^{\mathrm{a}}$. Fix two vectors $\hat{a}, \hat{b} \in \mathbb{C}^{\mathrm{a}}$ and, for $a \in A$, define $\zeta(a) \in \operatorname{Vect}(\Sigma)$ by

$$
\begin{aligned}
\zeta(a):= & \partial_{1} \eta(a, \hat{a}) \sqrt{-1} \hat{b}-j(a) \partial_{1} \eta(a, \hat{a}) \hat{b} \\
& -\partial_{1} \eta(a, \hat{b}) \sqrt{-1} \hat{a}+j(a) \partial_{1} \eta(a, \hat{b}) \hat{a}+[\eta(a, \hat{a}), \eta(a, \hat{b})] .
\end{aligned}
$$

Then

$$
\mathcal{L}_{\zeta(a)} j(a)=0, \quad \zeta(a)\left(r_{i}(a)\right)=0, \quad \mathcal{L}_{\zeta(a)} v(a)=0
$$

for $a \in A$ and $i=1, \ldots, \mathrm{n}$. Here the first equation follows from [4, Corrigendum, Lemma B] and the other two equations follow from similar, though somewhat lengthy, calculations. Now it follows from the stability condition in the definition of $\mathcal{P}$ that $\zeta(a)=0$ for every $a \in A$ and hence, by [4, Corrigendum, Lemma A] the almost complex structure $J_{P}$ is integrable. This proves the lemma.

4.5 Let $p_{0}:=\left(s_{0, *}, j_{0}, v_{0}\right) \in \mathcal{P}, B$ be a complex manifold with base point $b_{0} \in B$, and $\iota: B \rightarrow \mathcal{P}$ be a smooth map such that $\iota\left(b_{0}\right)=p_{0}$. The map $\iota$ is called holomorphic if its differential $d \iota(b): T_{b} B \rightarrow T_{\iota(b)} \mathcal{P}$ is complex linear for every $b \in B$. The map $\iota$ is called a slice at $b_{0}$ if for every smooth map $p:\left(A, a_{0}\right) \rightarrow\left(\mathcal{P}, p_{0}\right)$ there is a neighborhood $A_{0}$ of $a_{0}$ in $A$ and unique smooth maps $\Phi:\left(A_{0}, a_{0}\right) \rightarrow(\mathcal{G}$, id $)$ and $\phi:\left(A_{0}, a_{0}\right) \rightarrow\left(B, b_{0}\right)$ such that

$$
p(a)=\Phi(a)^{*} \iota(\phi(a))
$$

for $a \in A_{0}$. The map $\iota$ is called an infinitesimal slice at $b_{0}$ if

$$
\operatorname{im} d \iota\left(b_{0}\right) \oplus T_{p_{0}} \mathcal{G}^{*} p_{0}=T_{p_{0}} \mathcal{P}, \quad \operatorname{ker} d \iota\left(b_{0}\right)=0 .
$$

Write $\iota(b)=:\left(\sigma_{1}(b), \ldots, \sigma_{\mathrm{n}}(b), j(b), v(b)\right)$. Then (18) can be expressed as follows. 
$(\dagger)$ If $\hat{b} \in T_{b_{0}} B$ and $\hat{u} \in \operatorname{Vect}(\Sigma)$ satisfy

$$
\left.\begin{array}{r}
d \sigma_{\mathrm{i}}\left(b_{0}\right) \hat{b}-\hat{u}\left(s_{0, \mathrm{i}}\right)=0 \\
d j\left(b_{0}\right) \hat{b}+2 j_{0} \bar{\partial}_{j_{0}} \hat{u}=0 \\
d v\left(b_{0}\right) \hat{b}+d v_{0} \cdot \hat{u}=0
\end{array}\right\} \quad \Longrightarrow \quad \hat{b}=0, \hat{u}=0 .
$$

(†) If $\hat{s}_{\mathrm{i}} \in T_{s_{0, \mathrm{i}}} \Sigma, \hat{\jmath} \in T_{j_{0}} \mathcal{J}(\Sigma)$, and $\hat{v} \in \Omega^{0}\left(\Sigma, v_{0}^{*} T M\right)$ satisfy (8) then there exists a pair $(\hat{b}, \hat{u}) \in T_{b_{0}} B \times \operatorname{Vect}(\Sigma)$ such that

$$
\begin{aligned}
& d \sigma_{\mathrm{i}}\left(b_{0}\right) \hat{b}-\hat{u}\left(s_{0, \mathrm{i}}\right)=\hat{s}_{\mathrm{i}}, \\
& d j\left(b_{0}\right) \hat{b}+2 j_{0} \bar{\partial}_{j_{0}} \hat{u}=\hat{\jmath}, \\
& d v\left(b_{0}\right) \hat{b}+d v_{0} \cdot \hat{u}=\hat{v} .
\end{aligned}
$$

Theorem 4.6 (Slice Theorem) (i) A smooth infinitesimal slice is a slice.

(ii) If $\iota: B \rightarrow \mathcal{P}$ is an infinitesimal slice at $b_{0} \in B$ then it is an infinitesimal slice at $b$ for $b$ sufficiently near $b_{0}$.

(iii) Every point in $\mathcal{P}$ admits a holomorphic infinitesimal slice $\iota: B \rightarrow \mathcal{P}$ of complex dimension $\operatorname{dim}_{\mathbb{C}} B=(\mathrm{m}-3)(1-\mathrm{g})+\left\langle c_{1}, \mathrm{~d}\right\rangle+\mathrm{n}$.

Proof Choose an integer $s \geq 3$ and let $\mathcal{G}^{s}$ denote the Sobolev completion of $\mathcal{G}$ in the $H^{s}$ topology and $\mathcal{P}^{s}$ denote the Sobolev completion of $\mathcal{P}$ in the $H^{s-1}$ topology on $j$ and the $H^{s}$ topology on $v$. Then

$$
\mathcal{P}^{s} \subset \Sigma^{\mathrm{n}} \times \mathcal{J}^{s-1}(\Sigma) \times H^{s}(\Sigma, M)
$$

is a smooth Hilbert submanifold. Now let $\iota:\left(B, b_{0}\right) \rightarrow\left(\mathcal{P}, p_{0}\right)$ be a smooth infinitesimal slice.

Claim 1 The map

$$
B \times \mathcal{G}^{s} \rightarrow \mathcal{P}^{s}:(b, g) \mapsto \mathcal{F}^{s}(b, g):=g^{*} \iota(b)
$$

is a $C^{s-2}$ map between Hilbert manifolds. The tangent space of $\mathcal{G}^{s}$ at $\phi=$ id is the space $H^{s}(\Sigma, T \Sigma)$ of vector fields of class $H^{s}$ and the differential of $\mathcal{F}^{s}$ at the pair $(b$, id $)$ is

$$
d \mathcal{F}^{s}(b, \mathrm{id})(\hat{b}, \xi)=d \iota(b) \hat{b}+\mathcal{L}_{\iota(b)} \xi
$$

for $\hat{b} \in T_{b} B$ and $\xi \in H^{s}(\Sigma, T \Sigma)$. (See (11) for the definition of $\mathcal{L}_{l(b)}$.)

Denote the value of $\iota(b)$ at a point $x \in \Sigma$ by

$$
\iota(b)(x)=\left(\sigma_{1, b}, \ldots, \sigma_{\mathrm{n}, b}, j_{b}(x), v_{b}(x) .\right.
$$

The maps $\sigma_{i}: B \rightarrow \Sigma, j: B \times \Sigma \rightarrow \operatorname{End}(T \Sigma)$, and $v: B \times \Sigma \rightarrow M$ are all smooth by hypothesis. The map $\mathcal{G}^{s} \rightarrow \mathcal{G}^{s}: g \mapsto g^{-1}$ is smooth. Hence the map $B \times \mathcal{G}^{s} \rightarrow$ $\Sigma:(b, g) \mapsto g^{-1}\left(\sigma_{i, b}\right)$ is as smooth as the evaluation map $\mathcal{G}^{s} \times \Sigma \rightarrow \Sigma$, i.e. it is $C^{s-2}$ by Sobelov. Moreover, the map $g \mapsto d g$ is smooth as a map from $H^{s}$ to $H^{s-1}$. Since $\left(g^{*} j_{b}\right)(x)=d g(x)^{-1} j_{b}(g(x)) d g(x)$ this shows that the map

$$
B \times \mathcal{G}^{s} \rightarrow \mathcal{J}^{s-1}(\Sigma):(b, g) \mapsto g^{*} j_{b}
$$

is smooth. The map $B \times \mathcal{G}^{s} \rightarrow H^{s}(\Sigma, M):(b, g) \mapsto v_{b} \circ g$ is smooth because the map $v: B \times \Sigma \rightarrow M$ is smooth. This proves claim 1 . 
Claim 2 The operator $d \mathcal{F}^{s}(b$, id) is bijective if and only if $\iota$ is an infinitesimal slice at $b$.

To see this, assume first that $\iota$ is an infinitesimal slice at $b$. Then, by elliptic regularity, every element in the kernel of $d \mathcal{F}^{s}(b, \mathrm{id})$ is smooth and hence the operator is injective by ( $\left.\dagger\right)$. For surjectivity we observe that the image of $d \mathcal{F}^{S}(b$, id $)$ is closed by the elliptic estimate, that the smooth elements are dense in $T_{l(b)} \mathcal{P}^{s}$, and that the smooth elements of $T_{\iota(b)} \mathcal{P}^{s}$ are contained in the image of $d \mathcal{F}^{s}(b$, id) by ( $)$. Conversely, if $d \mathcal{F}^{s}(b$, id) is bijective, it follows from elliptic regularity that $\iota$ satisfies the infinitesimal slice conditions $(\dagger)$ and (†) at $b$. This proves claim 2 .

Shrinking $B$ if necessary, we may assume that $d \mathcal{F}^{s}(b, \mathrm{id})$ is bijective for every $b \in B$. By Claim 2 this implies that $\iota$ is an infinitesimal slice at every point $b \in B$ and $d \mathcal{F}^{s^{\prime}}$ (b, id) is bijective for every $b$ and every $s^{\prime}$. Hence, by equivariance, $d \mathcal{F}^{s^{\prime}}(b, g)$ is bijective for every integer $s^{\prime} \geq 2$, every $b \in B$, and every $g \in \mathcal{G}^{s^{\prime}}$. In particular, we have proved (ii).

Now fix an integer $s_{0} \geq 3$. Then it follows from the inverse function theorem that $\mathcal{F}^{s_{0}}$ maps an open $H^{s_{0}}$ neighborhood of $\left(b_{0}\right.$, id) in $B \times \mathcal{G}^{s_{0}}$ by a $C^{s_{0}-2}$-diffeomorphism onto an open neighborhood of $p_{0}$ in $\mathcal{P}^{s_{0}}$. Given a smooth map $p:\left(A, a_{0}\right) \rightarrow\left(\mathcal{P}, p_{0}\right)$ choose $A_{0} \subset A$ to be the preimage of this neighborhood of $p_{0}$ and define the $C^{s_{0}-2}$ map

$$
A_{0} \rightarrow B \times \mathcal{G}^{s_{0}}: a \mapsto(\phi(a), \Phi(a))
$$

by

$$
(\phi(a), \Phi(a)):=\left(\mathcal{F}^{s_{0}}\right)^{-1}(p(a))
$$

Then

$$
p(a)=\Phi(a)^{*} \iota(\phi(a))
$$

for every $a \in A_{0}$. Since the complex structures on $\Sigma$ associated to $\iota \circ \phi(a)$ and $p(a)$ are smooth it follows from elliptic regularity that $\Phi(a) \in \mathcal{G}$ is smooth for every $a \in A_{0}$. Thus $\Phi(a) \in \mathcal{G}^{s}$ and $\mathcal{F}^{s}(\phi(a), \Phi(a))=p(a)$ for every $a \in A_{0}$ and every $s$. Since the differential $d \mathcal{F}^{s}(\phi(a), \Phi(a))$ is bijective for every $a \in A_{0}$ and every integer $s \geq 2$, it follows that the map $a \mapsto(\phi(a), \Phi(a))$ is a $C^{s-2}$ map from $A_{0}$ to $B \times \mathcal{G}^{s}$ for every integer $s \geq 3$. Hence this map is smooth. This proves (i).

We prove (iii). Fix an element $\left(s_{0, *}, j_{0}, v_{0}\right) \in \mathcal{P}$. Let $\mathrm{G} \subset \mathcal{G}$ denote the identity component of the isotropy subgroup of the tuple $\left(s_{0, *}, j_{0}\right)$. Thus

$$
\mathrm{G}:= \begin{cases}\{11\}, & \text { if } \mathrm{n}>2-2 \mathrm{~g}, \\ \mathbb{T}^{2}, & \text { if } \mathrm{g}=1, \mathrm{n}=0, \\ \mathbb{C}^{*}, & \text { if } \mathrm{g}=0, \mathrm{n}=2, \\ \mathbb{C}^{*} \ltimes \mathbb{C}, & \text { if } \mathrm{g}=0, \mathrm{n}=1, \\ \operatorname{PSL}(2, \mathbb{C}), & \text { if } \mathrm{g}=0, \mathrm{n}=0 .\end{cases}
$$

First we choose a G-invariant holomorphic map

$$
\iota_{0}: A \rightarrow\left(\Sigma^{\mathrm{n}} \backslash \Delta\right) \times \mathcal{J}(\Sigma), \quad \iota_{0}(a)=\left(\sigma_{1}(a), \ldots, \sigma_{\mathrm{n}}(a), j(a)\right),
$$

defined on an open neighborhood $A \subset \mathbb{C}^{3 \mathrm{~g}-3+\mathrm{n}+\operatorname{dim}_{\mathbb{C}} \mathrm{G}}$ of a point $a_{0}$, that is transverse to the $\mathcal{G}$-action and satisfies

$$
\iota_{0}\left(a_{0}\right)=\left(s_{0, *}, j_{0}\right) .
$$

We do this as follows. In the case $n>2-2 \mathrm{~g}$ we choose a slice in Teichmüller space $\mathcal{T}_{\mathrm{g}, \mathrm{n}}$ as in the proof of [4, Theorem 8.9]. There are two cases with $n \leq 2-2 \mathrm{~g}$. If $\mathrm{g}=1$ (so $\Sigma \cong \mathbb{T}^{2}$ ) 
and $\mathrm{n}=0$ we take $A=\mathbb{H}$ to be the upper half plane and define $\iota_{0}: A \rightarrow \mathcal{J}(\Sigma)$ as the standard map to the complex structures on the torus (see [4, Sect. 7]). If $\mathrm{g}=0$ (so $\Sigma \cong S^{2}$ ) and $\mathrm{n} \leq 2$ we take $A$ to be a point. Note that

$$
\operatorname{dim}_{\mathbb{C}} A-\operatorname{dim}_{\mathbb{C}} \mathrm{G}=3 \mathrm{~g}-3+\mathrm{n}
$$

in all cases and that $\mathrm{G}$ is the isotropy group of each element of the slice, i.e. for $g \in \mathcal{G}$ and $a \in A$ we have $g^{*} \iota_{0}(a)=\iota_{0}(a)$ if and only if $g \in \mathrm{G}$.

The map $\iota_{0}$ gives rise to an infinite dimensional vector bundle

$$
\mathcal{E} \rightarrow A \times C^{\infty}(\Sigma, M)
$$

with fibers

$$
\mathcal{E}_{a, v}:=\Omega_{j(a)}^{0,1}\left(\Sigma, v^{*} T M\right) .
$$

The Cauchy-Riemann operator defines a section

$$
A \times C^{\infty}(\Sigma, M) \rightarrow \mathcal{E}:(a, v) \mapsto \bar{\partial}_{j(a), J}(v)
$$

whose intrinsic derivative at a point $(a, v)$ is the operator

$$
\mathcal{D}_{a, v}: T_{a} A \times \Omega^{0}\left(\Sigma, v^{*} T M\right) \rightarrow \Omega_{j(a)}^{0,1}\left(\Sigma, v^{*} T M\right)
$$

given by

$$
\mathcal{D}_{a, v}(\hat{a}, \hat{v}):=\mathcal{D}_{j(a), v}(\operatorname{dj}(a) \hat{a}, \hat{v})=D_{v} \hat{v}+\frac{1}{2} J(v) d v \cdot d j(a) \hat{a} .
$$

Since the operator $\mathcal{D}_{j_{0}, v_{0}}$ is surjective and $\iota_{0}$ is an infinitesimal slice, it follows that the Sect. (23) is transverse to the zero section at $\left(a_{0}, v_{0}\right)$. Hence it follows from the implicit function theorem in suitable Sobolev completions (see e.g. [2, Chap. 3]) that a neighborhood of $\left(a_{0}, v_{0}\right)$ in the zero set of (23) is a smooth submanifold of $A \times C^{\infty}(\Sigma, M)$. It is denoted by

$$
Z:=\left\{(a, v) \in A \times C^{\infty}(\Sigma, M) \mid \bar{\partial}_{j(a), J}(v)=0, \sup _{z \in \Sigma} d_{M}\left(v(z), v_{0}(z)\right)<\varepsilon\right\} .
$$

The group $\mathrm{G}$ acts on $Z$. Since

$$
\operatorname{index}_{\mathbb{R}}\left(D_{v}\right)=\mathrm{m}(2-2 \mathrm{~g})+2\left\langle c_{1}, \mathrm{~d}\right\rangle
$$

by the Riemann-Roch theorem, it follows from (22) that

$$
\operatorname{dim}_{\mathbb{R}} Z-\operatorname{dim}_{\mathbb{R}} \mathrm{G}=(\mathrm{m}-3)(2-2 \mathrm{~g})+2\left\langle c_{1}, \mathrm{~d}\right\rangle+2 \mathrm{n} .
$$

Since $\iota$ is holomorphic and $J$ is integrable, the operator (24) is complex linear for all $(a, v) \in$ $Z$. This shows that $Z$ is a finite dimensional submanifold of $A \times C^{\infty}(\Sigma, M)$ whose tangent space at each point $(a, v) \in Z$ is a complex subspace of $T_{a} A \times \Omega^{0}\left(\Sigma, v^{*} T M\right)$. The almost complex structure on any such submanifold is integrable, because $C^{\infty}(\Sigma, M)$ is a complex manifold and the graph of a smooth function between complex vector spaces is a complex submanifold if and only if the function is holomorphic. With this understood we obtain the desired infinitesimal slice from a holomorphic slice $B \subset Z$ for the $\mathrm{G}$ action. This proves the theorem.

Remark 4.7 In the proof of part (iii) of Theorem 4.6 one can reduce the case $\mathrm{n} \leq 2-2 \mathrm{~g}$ with $G \neq\{11\}$ to the case $n>2-2 \mathrm{~g}$ with $\mathrm{G}=\{11\}$ by a similar argument as we used in the proof of Theorem 3.5. 
4.8 Let $\left(s_{0, *}, j_{0}, v_{0}\right) \in \mathcal{P}, B$ be a manifold with base point $b_{0} \in B$, and

$$
B \rightarrow \mathcal{P}: b \mapsto \iota(b)=\left(\sigma_{1}(b), \ldots, \sigma_{\mathrm{n}}(b), j(b), v(b)\right)
$$

be a holomorphic map such that

$$
j\left(b_{0}\right)=j_{0}, \quad v\left(b_{0}\right)=v_{0}, \quad \sigma_{\mathrm{i}}\left(b_{0}\right)=s_{0, \mathrm{i}}, \quad \mathrm{i}=1, \ldots, \mathrm{n} .
$$

Define the unfolding $\left(\pi_{\iota}: Q_{\iota} \rightarrow B, S_{\iota, *}, H_{\iota}, b_{0}\right)$ by

$$
Q_{\iota}:=B \times \Sigma, \quad J_{\iota}(b, z):=\left(\begin{array}{cc}
\sqrt{-1} & 0 \\
0 & j(b)(z)
\end{array}\right)
$$

where $\sqrt{-1}$ denotes the complex structure on $B$ and

$$
H_{\iota}(b, z):=v(b)(z), \quad S_{\iota, \mathrm{i}}:=\left\{\left(b, \sigma_{\mathrm{i}}(b)\right) \mid b \in B\right\}, \quad \mathrm{i}=1, \ldots, \mathrm{n} .
$$

Lemma 4.9 Let $\left(\pi_{\iota}, S_{\iota, *}, H_{\iota}, b_{0}\right)$ be the unfolding associated to a holomorphic map $\iota: B \rightarrow$ $\mathcal{P}$ as in 4.8. Then the following are equivalent.

(i) The unfolding $\left(\pi_{\iota}, S_{\iota, *}, H_{\iota}, b_{0}\right)$ is infinitesimally universal.

(ii) The map $\iota$ is an infinitesimal slice at $b_{0}$.

Proof Let $u_{0}:\left(\Sigma, j_{0}\right) \rightarrow Q_{\iota}$ be the holomorphic embedding

$$
u_{0}(z):=\left(b_{0}, z\right)
$$

so that $H_{\iota} \circ u_{0}=v_{0}$. Then the operator $D_{u_{0}}$ has domain

$$
\mathcal{X}_{u}:=\left\{(\hat{u}, \hat{b}) \in \Omega^{0}(\Sigma, T \Sigma) \times T_{b_{0}} B \mid \hat{u}\left(s_{0, \mathrm{i}}\right)=d \sigma_{\mathrm{i}}\left(b_{0}\right) \hat{b}\right\},
$$

target space $\mathcal{Y}_{u}:=\Omega_{j_{0}}^{0,1}(\Sigma, T \Sigma)$, and is given by

$$
D_{u_{0}}(\hat{u}, \hat{b})=\bar{\partial}_{j_{0}} \hat{u}-\frac{1}{2} j_{0} d j\left(b_{0}\right) \hat{b} .
$$

The linearized operator in 2.6 is

$$
D_{v_{0}}: \mathcal{X}_{v} \rightarrow \mathcal{Y}_{v}, \quad \mathcal{X}_{v}:=\Omega^{0}\left(\Sigma, v_{0}^{*} T M\right), \quad \mathcal{Y}_{v}:=\Omega^{0,1}\left(\Sigma, v_{0}^{*} T M\right)
$$

The homomorphisms

$$
\operatorname{ker} D_{u_{0}} \rightarrow \operatorname{ker} D_{v_{0}}, \quad \operatorname{coker} D_{u_{0}} \rightarrow \operatorname{coker} D_{v_{0}}
$$

are induced by the maps

$$
\mathcal{X}_{u} \rightarrow \mathcal{X}_{v}:(\hat{u}, \hat{b}) \mapsto d v_{0} \cdot \hat{u}+d v\left(b_{0}\right) \hat{b}, \quad \mathcal{Y}_{u} \rightarrow \mathcal{Y}_{v}: \eta \mapsto d v_{0} \cdot \eta
$$

We must prove that the maps in (25) are isomorphisms if and only if (ii) holds. Note that the second map in (25) is necessarily surjective because $\left(\Sigma, s_{0, *}, j_{0}, v_{0}\right)$ is a regular stable map.

We prove that (ii) implies (i). We prove that the first map in (25) is bijective. Let $(\hat{u}, \hat{b}) \in$ ker $D_{u_{0}}$ and assume that its image in ker $D_{v_{0}}$ vanishes. Then

$$
\bar{\partial}_{j_{0}} \hat{u}-\frac{1}{2} j_{0} d j\left(b_{0}\right) \hat{b}=0, \quad d v_{0} \cdot \hat{u}+d v\left(b_{0}\right) \hat{b}=0 .
$$

Since $(\hat{u}, \hat{b}) \in \mathcal{X}_{u}$, we have $d \sigma_{\mathrm{i}}\left(b_{0}\right)=\hat{u}\left(s_{0, \mathrm{i}}\right)$ for $\mathrm{i}=1, \ldots, \mathrm{n}$ and hence, by (ii) and $(\dagger)$ in $4.5, \hat{b}=0$ and $\hat{u}=0$. Thus we have proved that the homomorphism $\operatorname{ker} D_{u_{0}} \rightarrow \operatorname{ker} D_{v_{0}}$ 
is injective. Next we prove that this map is surjective. Let $\hat{v} \in \Omega^{0}\left(\Sigma, v_{0}^{*} T M\right)$ be a vector field along $v_{0}$ such that $D_{v_{0}} \hat{v}=0$. Then the tuple $\left(\hat{s}_{1}, \ldots \hat{s}_{\mathrm{n}}, \hat{\jmath}, \hat{v}\right)$ with $\hat{s}_{\mathrm{i}}=0$ and $\hat{\jmath}=0$ satisfies (8). Hence, by (ii) and (†) in 4.5 , there is a pair $(\hat{u}, \hat{b})$ such that

$$
d \sigma_{\mathrm{i}}\left(b_{0}\right) \hat{a}-\hat{u}\left(s_{0, \mathrm{i}}\right)=0, \quad d j\left(b_{0}\right) \hat{b}+2 j_{0} \bar{\partial}_{j_{0}} \hat{u}=0, \quad d v\left(b_{0}\right) \hat{b}+d v_{0} \cdot \hat{u}=\hat{v} .
$$

This implies

$$
\bar{\partial}_{j_{0}} \hat{u}-\frac{1}{2} j_{0} d j\left(b_{0}\right) \hat{b}=0, \quad \hat{v}=d v\left(b_{0}\right) \hat{b}+d v_{0} \cdot \hat{u}
$$

and so $\hat{v}$ belongs to the image of the map ker $D_{u_{0}} \rightarrow \operatorname{ker} D_{v_{0}}$. This shows that the first map in (25) is an isomorphism.

Next we prove that the second map in (25) is bijective. Let $\eta \in \mathcal{Y}_{u}$ such that $d v_{0} \cdot \eta \in \operatorname{im} D_{v_{0}}$ and choose $\hat{v} \in \Omega^{0}\left(\Sigma, v_{0}^{*} T M\right)$ such that

$$
d v_{0} \cdot \eta+D_{v_{0}} \hat{v}=0 .
$$

Then $\hat{v}$ and $\hat{\jmath}:=-2 j_{0} \eta$ satisfy (8). Hence, by (ii) and $(\ddagger)$ in 4.5 , there is a pair $(\hat{u}, \hat{b})$ such that

$$
d \sigma_{\mathrm{i}}\left(b_{0}\right) \hat{b}-\hat{u}\left(s_{0, \mathrm{i}}\right)=0, \quad d j\left(b_{0}\right) \hat{b}+2 j_{0} \bar{\partial}_{j_{0}} \hat{u}=\hat{\jmath}, \quad d v\left(b_{0}\right) \hat{b}+d v_{0} \cdot \hat{u}=\hat{v} .
$$

This implies

$$
(\hat{u}, \hat{b}) \in \mathcal{X}_{u}, \quad D_{u_{0}}(\hat{u}, \hat{b})=-\frac{1}{2} j_{0} \hat{\jmath}=-\eta,
$$

and hence $\eta \in \operatorname{im} D_{u_{0}}$. This shows that the second map in (25) is injective and, since we have already proved surjectivity, it is an isomorphism. Thus we have proved that (ii) implies (i).

We prove that (i) implies (ii). Assume that the maps in (25) are bijective. If $\hat{u}$ and $\hat{b}$ satisfy (19) then $(\hat{u}, \hat{b}) \in \mathcal{X}_{u}, D_{u_{0}}(\hat{u}, \hat{b})=0$, and the image of $(\hat{u}, \hat{b})$ under the homomorphism $\mathcal{X}_{u} \rightarrow \mathcal{X}_{v}$ vanishes. Since the first map in (25) is injective, this implies $\hat{u}=0$ and $\hat{b}=0$. Now suppose that $\hat{\jmath}$ and $\hat{v}$ satisfy (8) with $v=v_{0}$, i.e.

$$
0=D_{v_{0}} \hat{v}+\frac{1}{2} J\left(v_{0}\right) d v_{0} \circ \hat{\jmath}=D_{v_{0}} \hat{v}+d v_{0} \circ \eta, \quad \eta:=\frac{1}{2} j_{0} \hat{\jmath} .
$$

Hence $d v_{0} \circ \eta=-D_{v_{0}} \hat{v} \in \operatorname{im} D_{v_{0}}$. Since the second map in (25) is injective this implies $\eta \in \operatorname{im} D_{u_{0}}$. Choose a pair $(\hat{u}, \hat{b}) \in \mathcal{X}_{u}$ such that $D_{u_{0}}(\hat{u}, \hat{b})=-\eta$. Then $\hat{u}$ and $\hat{b}$ satisfy

$$
d \sigma_{\mathrm{i}}\left(b_{0}\right) \hat{b}-\hat{u}\left(s_{0, \mathrm{i}}\right)=0, \quad \hat{\jmath}=-2 j_{0} \eta=2 j_{0} D_{u_{0}}(\hat{u}, \hat{b})=2 j_{0} \bar{\partial}_{j_{0}} \hat{u}+\operatorname{dj}\left(b_{0}\right) \hat{b} .
$$

Hence

$$
D_{v_{0}} \hat{v}=-d v_{0} \cdot \eta=d v_{0} \cdot D_{u_{0}}(\hat{u}, \hat{b})=D_{v_{0}}\left(d v_{0} \cdot \hat{u}+d v\left(b_{0}\right) \hat{b}\right) .
$$

The last equation follows from the fact that and the diagram (5) in Definition 3.3 commutes, reading $H_{\iota}(p, z)=v(b)(z)$ for $H$. Since the first map in (25) is surjective, there exists a pair $\left(\hat{u}_{0}, \hat{b}_{0}\right) \in \operatorname{ker} D_{u_{0}}$ such that

$$
\hat{v}=d v_{0} \cdot\left(\hat{u}+\hat{u}_{0}\right)+d v\left(b_{0}\right)\left(\hat{b}+\hat{b}_{0}\right) .
$$

Hence the pair $\left(\hat{u}+\hat{u}_{0}, \hat{b}+\hat{b}_{0}\right)$ satisfies (20) with $\hat{s}_{\mathrm{i}}=0$. In the case $\hat{s}_{\mathrm{i}} \neq 0$ choose first a vector field $\hat{u}_{0} \in \operatorname{Vect}(\Sigma)$ such that $-\hat{u}_{0}\left(s_{0, \mathrm{i}}\right)=\hat{s}_{\mathrm{i}}$ for $\mathrm{i}=1, \ldots, \mathrm{n}$ and denote

$$
\hat{\jmath}_{1}:=\hat{\jmath}-2 j_{0} \bar{\partial}_{j_{0}} \hat{u}_{0}, \quad \hat{v}_{1}:=\hat{v}-d v_{0} \cdot \hat{u}_{0} .
$$


This pair still satisfies (8). Hence, by what we have already proved, there exists a pair $\left(\hat{u}_{1}, \hat{b}_{1}\right)$ that satisfies (20) with $\left(\hat{s}_{\mathrm{i}}, \hat{\jmath}, \hat{v}\right)$ replaced by $\left(0, \hat{\jmath}_{1}, \hat{v}_{1}\right)$. Hence the pair $\hat{u}:=\hat{u}_{0}+\hat{u}_{1}, \hat{b}:=\hat{b}_{1}$ satisfies (20). Thus we have proved that (i) implies (ii). This completes the proof of the lemma.

Lemma 4.10 Fix a regular stable map $\left(\Sigma, s_{0, *}, j_{0}, v_{0}\right)$ and let

$$
B \rightarrow \mathcal{P}: b \mapsto \iota(b)=\left(\sigma_{1}(b), \ldots, \sigma_{\mathrm{n}}(b), j(b), v(b)\right)
$$

be a holomorphic infinitesimal slice such that

$$
\iota\left(b_{0}\right)=\left(s_{0, *}, j_{0}, v_{0}\right) .
$$

Let $\left(\pi_{\iota}, S_{\iota, *}, H_{l}, b_{0}\right)$ be the unfolding constructed in 4.8 . Then every continuously differentiable morphism $(\phi, \Phi)$ from $\left(\pi_{A}: P \rightarrow A, R_{*}, H_{A}, a_{0}\right)$ to $\left(\pi_{\iota}, S_{\iota, *}, H_{\iota}, b_{0}\right)$ is holomorphic.

Proof Choose a smooth trivialization

$$
A \times \Sigma \rightarrow P:(a, z) \mapsto \tau(a, z)=\tau_{a}(z)
$$

so that $\tau_{a}: \Sigma \rightarrow P_{a}$ is a desingularization (with no singularities) for every $a \in A$. The stable map on $\Sigma$, induced by $\tau_{a}$, is the tuple

$$
p(a):=\iota \circ \phi(a)=\left(\sigma_{1}(\phi(a)), \ldots, \sigma_{\mathrm{n}}(\phi(a)), j(\phi(a)), v(\phi(a))\right) \in \mathcal{P} .
$$

The complex structure on $A \times \Sigma$ induced by $\tau$ has the form

$$
(\hat{a}, \hat{z}) \mapsto(\sqrt{-1} \hat{a}, j(\phi(a))(z) \hat{z}+\eta(a, \hat{a})(z))
$$

for a suitable 1-form $T_{a} A \rightarrow \operatorname{Vect}(\Sigma): \hat{a} \mapsto \eta(a, \hat{a})$. Since this complex structure is integrable, the map $H_{A} \circ \tau: A \times \Sigma \rightarrow M$ is holomorphic, and $\tau^{-1}\left(R_{\mathrm{i}}\right)$ is a complex submanifold of $A \times \Sigma$ for every i, it follows from Lemma 4.4 that

$$
d p(a) \hat{a}+\mathcal{I}(p(a)) d p(a) \sqrt{-1} \hat{a}-\mathcal{L}_{p(a)} \eta(a, \sqrt{-1} \hat{a})=0
$$

for every $a \in A$ and every $\hat{a} \in T_{a} A$. Since $p=\iota \circ \phi$ and $\iota$ is holomorphic, this implies

$$
d \iota(\phi(a))(d \phi(a) \hat{a}+\sqrt{-1} d \phi(a) \sqrt{-1} \hat{a})=\mathcal{L}_{l(\phi(a))} \eta(a, \sqrt{-1} \hat{a})
$$

for all $a$ and $\hat{a}$. Since $\iota$ is a slice this implies that $\eta \equiv 0$ and $\phi$ is holomorphic. Hence $\Phi$ is holomorphic as well and this proves the lemma.

Theorem 4.11 Theorems 3.5,3.6, and 3.8 holdfor regular stable maps without nodes. Moreover, if $\left(\pi_{B}: Q \rightarrow B, S_{*}, H_{B}, b_{0}\right)$ is any universal unfolding without nodes and $(\phi, \Phi)$ is a continuously differentiable morphism from $\left(\pi_{A}: P \rightarrow A, R_{*}, H_{A}, a_{0}\right)$ to $\left(\pi_{B}, S_{*}, H_{B}, b_{0}\right)$ then $\phi$ and $\Phi$ are holomorphic.

Proof Step 1. Theorem 3.5 holds for stable maps without nodes. We proved "only if" immediately after the statement of Theorem 3.5; we prove "if" here. Fix a regular stable map $\left(\Sigma, s_{0, *}, j_{0}, v_{0}\right)$, let $\iota: B \rightarrow \mathcal{P}$ be a holomorphic infinitesimal slice such that $\iota\left(b_{0}\right)=\left(s_{0, *}, j_{0}, v_{0}\right)$, and let $\left(\pi_{\iota}, S_{\iota, *}, H_{\iota}, b_{0}\right)$ be the unfolding constructed in 4.8 . Then it follows from Lemma 4.9 that $\left(\pi_{\iota}, S_{\iota, *}, H_{\iota}, b_{0}\right)$ is infinitesimally universal.

Step 2. The unfolding $\left(\pi_{\iota}, S_{\iota, *}, H_{\iota}, b_{0}\right)$ is universal. Let $\left(\pi_{A}, R_{*}, H_{A}, a_{0}\right)$ be an unfolding of $\left(\Sigma, s_{0, *}, j_{0}, v_{0}\right)$ and $f_{0}: P_{a_{0}} \rightarrow Q_{b_{0}}$ be a fiber isomorphism. Assume w.l.o.g. that

$$
P=A \times \Sigma, \quad f_{0}\left(a_{0}, z\right)=\left(b_{0}, z\right) .
$$


Denote by $p(a)=\left(r_{*}(a), j(a), v(a)\right) \in \mathcal{P}$ the regular stable map on the fiber over $a$ determined by $\left(\pi_{A}, R_{*}, H_{A}, a_{0}\right)$. Then

$$
p\left(a_{0}\right)=\left(s_{0, *}, j_{0}, v_{0}\right)=\iota\left(b_{0}\right) .
$$

Now any two smooth maps $\phi: A \rightarrow B$ and $\Phi: P \rightarrow Q_{\iota}$ that intertwine the projections and satisfy $\Phi \mid P_{a_{0}}=f_{0}$ have the form

$$
\Phi(a, z)=\left(\phi(a), \Phi_{a}(z)\right),
$$

where $A \rightarrow \operatorname{Diff}(\Sigma): a \mapsto \Phi_{a}$ is a smooth map such that $\Phi_{a_{0}}=$ id. The pair $(\phi, \Phi)$ is a smooth morphism from $\left(\pi_{A}, R_{*}, H_{A}, a_{0}\right)$ to $\left(\pi_{\iota}, S_{\iota, *}, H_{\iota}, b_{0}\right)$ if and only if

$$
p(a)=\Phi_{a}^{*} \iota(\phi(a))
$$

for every $a \in A$. Hence the existence and uniqueness of smooth morphisms follows from the Theorem 4.6 (i). That every smooth morphism is holomorphic follows from Lemma 4.10.

Step 3. Every infinitesimally universal unfolding of $\left(\Sigma, s_{0, *}, j_{0}, v_{0}\right)$ is isomorphic to $\left(\pi_{\iota}, S_{\iota, *}, H_{\iota}, b_{0}\right)$. Let $\left(\pi_{A}, R_{*}, H_{A}, a_{0}\right)$ be an unfolding and

$$
f_{0}: P_{a_{0}} \rightarrow Q_{b_{0}}
$$

be a fiber isomorphism. By Step 2, there exists a holomorphic morphism $(\phi, \Phi)$ from $\left(\pi_{A}, R_{*}, H_{A}, a_{0}\right)$ to $\left(\pi_{\iota}, S_{\iota, *}, H_{\iota}, b_{0}\right)$. The map

$$
p:=\iota \circ \phi: A \rightarrow \mathcal{P}
$$

is holomorphic. Since $\left(\pi_{A}, R_{*}, H_{A}, a_{0}\right)$ is infinitesimally universal, $p$ is a infinitesimal slice at $a_{0}$, by Lemma 4.9. Hence the differential $d \phi\left(a_{0}\right)$ is bijective. This implies that $(\phi, \Phi)$ is an isomorphism.

Step 4. Since every infinitesimally universal unfolding of $\left(\Sigma, s_{0, *}, j_{0}, v_{0}\right)$ is isomorphic to $\left(\pi_{\iota}, S_{\iota, *}, H_{\iota}, b_{0}\right)$ and $\left(\pi_{\iota}, S_{\iota, *}, H_{\iota}, b_{0}\right)$ is universal we have proved Theorem 3.6 for stable maps without nodes. By Lemma 4.9 and Theorem 4.6, the unfolding $\left(\pi_{\iota}, S_{\iota, *}, H_{\iota}, b\right)$ is infinitesimally universal for $b$ near $b_{0}$ and hence Theorem 3.8 holds for stable maps without nodes. The 'moreover' assertion follows from Lemma 4.10 and Step 3. This proves Theorem 4.11.

\section{Hardy decompositions}

This section follows closely Sects. 9 and 11 of [4]. It is convenient to use slightly different notation; for example $P=N \cup M$ in [4] becomes $P=P^{\prime} \cup P^{\prime \prime}$ and the open sets $U, V \subset Q$ in [4] are replaced by $U^{\prime}, U^{\prime \prime}$. With these changes we review the notation from [4].

5.1 Throughout this section

$$
\left(\pi_{A}: P \rightarrow A, R_{*}, H_{A}, a_{0}\right), \quad\left(\pi_{B}: Q \rightarrow B, S_{*}, H_{B}, b_{0}\right)
$$

are unfoldings of maps,

$$
f_{0}: P_{a_{0}} \rightarrow Q_{b_{0}}
$$

is a fiber isomorphism, and $p_{1}, p_{2}, \ldots, p_{\mathrm{k}}$ are the nodal points of the central fiber $P_{a_{0}}$, so $q_{\mathrm{i}}:=f_{0}\left(p_{\mathrm{i}}\right)$ (for $\left.\mathrm{i}=1, \ldots, \mathrm{k}\right)$ are the nodal points of the central fiber $Q_{b_{0}}$. As in [4] we denote by $C_{A} \subset P$ and $C_{B} \subset Q$ the critical points of $\pi_{A}$ and $\pi_{B}$, respectively. 
5.2 Let $U^{\prime} \subset Q$ be an open neighborhood of $C_{B}$ equipped with nodal coordinates. This means

$$
U^{\prime}=U_{1}^{\prime} \cup \cdots \cup U_{\mathrm{k}}^{\prime}
$$

where the sets $U_{\mathrm{i}}^{\prime}$ have pairwise disjoint closures, each $U_{\mathrm{i}}^{\prime}$ is a connected neighborhood of one of the components of $C_{B}$, and for $\mathrm{i}=1, \ldots, \mathrm{k}$ there is a holomorphic coordinate system

$$
\left(\zeta_{\mathrm{i}}, \tau_{\mathrm{i}}\right): B \rightarrow \mathbb{C} \times \mathbb{C}^{\mathrm{b}-1}
$$

and holomorphic functions $\xi_{\mathrm{i}}, \eta_{\mathrm{i}}: U_{\mathrm{i}}^{\prime} \rightarrow \mathbb{C}$ such that

$$
\left(\xi_{\mathrm{i}}, \eta_{\mathrm{i}}, \tau_{\mathrm{i}} \circ \pi_{B}\right): U_{\mathrm{i}}^{\prime} \rightarrow \mathbb{C} \times \mathbb{C} \times \mathbb{C}^{\mathrm{b}-1}
$$

is a holomorphic coordinate system and $\xi_{\mathrm{i}} \eta_{\mathrm{i}}=\zeta_{\mathrm{i}} \circ \pi_{B}$. Assume that $\bar{U}^{\prime} \cap S_{*}=\varnothing$. Let $U^{\prime \prime} \subset Q$ be an open set such that

$$
Q=U^{\prime} \cup U^{\prime \prime}, \quad \bar{U}^{\prime \prime} \cap C_{B}=\emptyset,
$$

and $U_{\mathrm{i}}^{\prime} \cap U^{\prime \prime}$ intersects each fiber $Q_{b}$ in two open annuli with $\left|\xi_{\mathrm{i}}\right|>\left|\eta_{\mathrm{i}}\right|$ on one component and $\left|\xi_{\mathrm{i}}\right|<\left|\eta_{\mathrm{i}}\right|$ on the other. Introduce the abbreviations

$$
\begin{gathered}
U:=U^{\prime} \cap U^{\prime \prime}, \quad U_{\mathrm{i}}:=U_{\mathrm{i}}^{\prime} \cap U^{\prime \prime}, \quad U_{\mathrm{i}, 1}:=\left\{\left|\xi_{\mathrm{i}}\right|>\left|\eta_{\mathrm{i}}\right|\right\}, \quad U_{\mathrm{i}, 2}:=\left\{\left|\xi_{\mathrm{i}}\right|<\left|\eta_{\mathrm{i}}\right|\right\}, \\
U_{b}^{\prime}:=U^{\prime} \cap Q_{b}, \quad U_{b}^{\prime \prime}:=U^{\prime \prime} \cap Q_{b}, \quad U_{b}:=U \cap Q_{b} .
\end{gathered}
$$

5.3 As in [4] we use a Hardy decomposition (Fig. 1)

$$
P=P^{\prime} \cup P^{\prime \prime}, \quad \partial P^{\prime}=\partial P^{\prime \prime}=P^{\prime} \cap P^{\prime \prime},
$$

for $\left(\pi_{A}, R_{*}, a_{0}\right)$. Thus $P^{\prime}$ and $P^{\prime \prime}$ are submanifolds of $P$ intersecting in their common boundary and

$$
P^{\prime}=P_{1}^{\prime} \cup \cdots \cup P_{\mathrm{k}}^{\prime}
$$

Fig. 1 Hardy decomposition of $P$
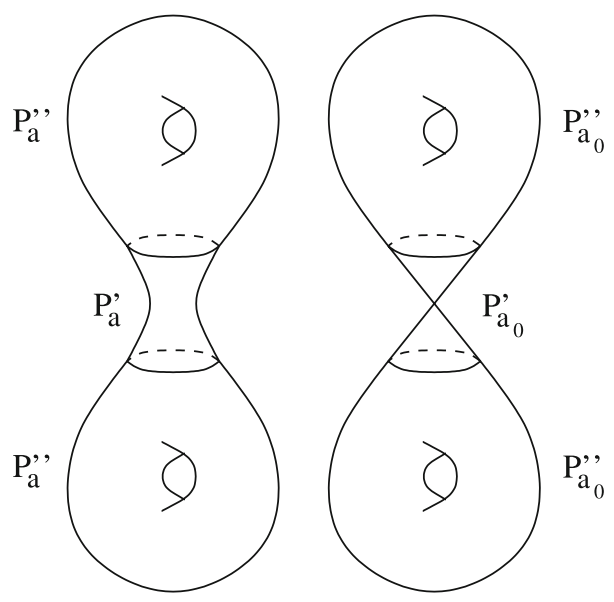
where $P_{\mathrm{i}}^{\prime}$ is a closed neighborhood of $p_{\mathrm{i}}$ disjoint from the elements of $R_{*}$, the $P_{\mathrm{i}}^{\prime}$ are pairwise disjoint, and each $P_{\mathrm{i}}^{\prime}$ is the domain of a nodal coordinate system. The latter consists of three holomorphic maps

$$
\left(x_{\mathrm{i}}, y_{\mathrm{i}}\right): P_{\mathrm{i}}^{\prime} \rightarrow \mathbb{D}^{2}, \quad z_{\mathrm{i}}: A \rightarrow \mathbb{C}, \quad t_{\mathrm{i}}: A \rightarrow \mathbb{C}^{\mathrm{a}-1},
$$

such that each map

$$
A \rightarrow \mathbb{D} \times \mathbb{C}^{\mathrm{a}-1}: a \mapsto\left(z_{\mathrm{i}}(a), t_{\mathrm{i}}(a)\right)
$$

is a holomorphic coordinate system, each map

$$
P_{\mathrm{i}}^{\prime} \rightarrow \mathbb{D}^{2} \times \mathbb{C}^{\mathrm{a}-1}: p \mapsto\left(x_{\mathrm{i}}(p), y_{\mathrm{i}}(p), t_{\mathrm{i}}\left(\pi_{A}(p)\right)\right)
$$

is a holomorphic coordinate system, and

$$
x_{\mathrm{i}}\left(p_{\mathrm{i}}\right)=y_{\mathrm{i}}\left(p_{\mathrm{i}}\right)=0, \quad z_{\mathrm{i}} \circ \pi_{A}=x_{\mathrm{i}} y_{\mathrm{i}} .
$$

Restricting to a fiber gives a decomposition

$$
P_{a}=P_{a}^{\prime} \cup P_{a}^{\prime \prime}, \quad P_{a}^{\prime}:=P^{\prime} \cap P_{a}, \quad P_{a}^{\prime \prime}:=P^{\prime \prime} \cap P_{a},
$$

where $P_{a}^{\prime \prime}$ is a Riemann surface with boundary and each component of $P_{a}^{\prime}$ is either a closed annulus or a pair of transverse closed disks. Abbreviate

$$
\Gamma_{a}:=P_{a}^{\prime} \cap P_{a}^{\prime \prime}=\partial P_{a}^{\prime}=\partial P_{a}^{\prime \prime} .
$$

The nodal coordinate system determines a trivialization

$$
\iota: A \times \Gamma \rightarrow \partial P^{\prime}, \quad \Gamma:=\bigcup_{\mathrm{i}=1}^{\mathrm{k}}\{(\mathrm{i}, 1),(\mathrm{i}, 2)\} \times S^{1},
$$

given by

$$
\begin{array}{ll}
\iota^{-1}(p):=\left(\pi_{A}(p),(\mathrm{i}, 1), x_{\mathrm{i}}(p)\right), & p \in \partial_{1} P_{\mathrm{i}}^{\prime}:=\left\{\left|x_{\mathrm{i}}\right|=1\right\}, \\
\iota^{-1}(q):=\left(\pi_{A}(p),(\mathrm{i}, 2), y_{\mathrm{i}}(q)\right), & q \in \partial_{2} P_{\mathrm{i}}^{\prime}:=\left\{\left|y_{\mathrm{i}}\right|=1\right\} .
\end{array}
$$

For $a \in A$ and $\mathrm{i}=1, \ldots, \mathrm{k}$ define $\iota_{a}: \Gamma \rightarrow \Gamma_{a}$ by $\iota_{a}(\lambda):=\iota(a, \lambda)$ and denote

$$
\partial_{\mathrm{i}, 1} P_{a}^{\prime}:=\partial_{1} P_{\mathrm{i}}^{\prime} \cap P_{a}, \quad \partial_{\mathrm{i}, 2} P_{a}^{\prime}:=\partial_{2} P_{\mathrm{i}}^{\prime} \cap P_{a}, \quad P_{a, \mathrm{i}}^{\prime}:=P_{a}^{\prime} \cap P_{\mathrm{i}}^{\prime} .
$$

5.4 Lemma 11.3 in [4] asserts that, after shrinking $A$ and $B$ if necessary, there is a Hardy decomposition $P=P^{\prime} \cup P^{\prime \prime}$ as in 5.3 and there are open subsets $U^{\prime}=U_{1}^{\prime} \cup \cdots \cup U_{\mathrm{k}}^{\prime}, U^{\prime \prime}$, $U$ of $Q$ and functions $\xi_{\mathrm{i}}, \eta_{\mathrm{i}}, \zeta_{\mathrm{i}}, \tau_{\mathrm{i}}$ as described in 5.2 such that

$$
\begin{aligned}
& f_{0}\left(P_{a_{0}}^{\prime}\right) \subset U_{b_{0}}^{\prime}, \quad f_{0}\left(P_{a_{0}}^{\prime \prime}\right) \subset U_{b_{0}}^{\prime \prime}, \\
& \xi_{\mathrm{i}} \circ f_{0} \circ x_{\mathrm{i}}^{-1}(x, 0,0)=x, \quad \eta_{\mathrm{i}} \circ f_{0} \circ y_{\mathrm{i}}^{-1}(0, y, 0)=y
\end{aligned}
$$

for $x, y \in \mathbb{D}$. Fix a Hardy decomposition $P=P^{\prime} \cup P^{\prime \prime}$ for $\left(\pi_{A}, R_{*}, a_{0}\right)$, open subsets $U^{\prime}=U_{1}^{\prime} \cup \cdots \cup U_{\mathrm{k}}^{\prime}, U^{\prime \prime}, U$ of $Q$, and functions $\xi_{\mathrm{i}}, \eta_{\mathrm{i}}, \zeta_{\mathrm{i}}, \tau_{\mathrm{i}}$ as described in 5.2, such that these conditions are satisfied.

5.5 Fix an integer $s+1 / 2>1$. For $a \in A$ and $b \in B$ define an open subset

$$
\mathcal{U}(a, b) \subset H^{s}\left(\Gamma_{a}, U_{b}\right)
$$


by the condition that for $\alpha \in H^{s}\left(\Gamma_{a}, U_{b}\right)$ we have $\alpha \in \mathcal{U}(a, b)$ if

$$
\alpha\left(\partial_{\mathrm{i}, 1} P_{a}^{\prime}\right) \subset U_{\mathrm{i}, 1}, \quad \alpha\left(\partial_{\mathrm{i}, 2} P_{a}^{\prime}\right) \subset U_{\mathrm{i}, 2},
$$

(see 5.2 for the notation $U_{\mathrm{i}, 1}$ and $U_{\mathrm{i}, 2}$ ) and the curves $\xi_{\mathrm{i}} \circ \alpha \circ x_{\mathrm{i}}^{-1}$ and $\eta_{\mathrm{i}} \circ \alpha \circ y_{\mathrm{i}}^{-1}$ from $S^{1}$ to $\mathbb{C} \backslash 0$ both have winding number one about the origin.

$$
\begin{gathered}
\mathcal{U}^{\prime}(a, b):=\left\{\begin{array}{l|l}
\alpha \in \mathcal{U}(a, b) & \begin{array}{l}
\exists f^{\prime} \in \mathrm{Hol}^{s+1 / 2}\left(P_{a}^{\prime}, U_{b}^{\prime}\right): \alpha=f^{\prime} \mid \Gamma_{a} \\
\text { and } f^{\prime}\left(C_{A} \cap P_{a}\right)=C_{B} \cap Q_{b},
\end{array}
\end{array}\right\}, \\
\mathcal{U}^{\prime \prime}(a, b):=\left\{\begin{array}{l|l}
\alpha \in \mathcal{U}(a, b) & \begin{array}{l}
\exists f^{\prime \prime} \in \mathrm{Hol}^{s+1 / 2}\left(P_{a}^{\prime \prime}, U_{b}^{\prime \prime}\right): \alpha=f^{\prime \prime} \mid \Gamma_{a} \\
\text { and } f^{\prime \prime}\left(R_{*} \cap P_{a}\right)=S_{*} \cap Q_{b}
\end{array}
\end{array}\right\} .
\end{gathered}
$$

Here $\mathrm{Hol}^{s+1 / 2}(X, Y)$ denotes the set of maps of class $H^{s+1 / 2}$ from $X$ to $Y$ which are holomorphic on the interior of $X$. Holomorphicity at a nodal point is defined as in [4, Sect. 11.1]. Note that the function $f^{\prime}: P_{a}^{\prime} \rightarrow U_{b}^{\prime}$ in the definition of $\mathcal{U}^{\prime}(a, b)$ maps the boundary $\Gamma_{a}=\partial P_{a}^{\prime}$ into $U_{b}=U_{b}^{\prime} \cap U_{b}^{\prime \prime}$; similarly for $f^{\prime \prime}$ in the definition of $\mathcal{U}^{\prime \prime}(a, b)$. Define

$$
\begin{gathered}
\mathcal{U}_{a}:=\bigsqcup_{b \in B} \mathcal{U}(a, b), \quad \mathcal{U}_{a}^{\prime}:=\bigsqcup_{b \in B} \mathcal{U}^{\prime}(a, b), \quad \mathcal{U}_{a}^{\prime \prime}:=\bigsqcup_{b \in B} \mathcal{U}^{\prime \prime}(a, b), \\
\mathcal{U}:=\bigsqcup_{a \in A} \mathcal{U}_{a}, \quad \mathcal{U}^{\prime}:=\bigsqcup_{a \in A} \mathcal{U}_{a}^{\prime}, \quad \mathcal{U}^{\prime \prime}:=\bigsqcup_{a \in A} \mathcal{U}_{a}^{\prime \prime} .
\end{gathered}
$$

Our notation means that the three formulas $(a, \alpha, b) \in \mathcal{U},(\alpha, b) \in \mathcal{U}_{a}$, and $\alpha \in \mathcal{U}(a, b)$ have the same meaning.

5.6 We use the nodal coordinate system of 5.3 to construct an auxiliary Hilbert manifold structure on $\mathcal{U}$. The domains of the maps in this space vary with $a$ so we replace them with a constant domain by using an appropriate trivialization. Define an open set

$$
\mathcal{U}_{0} \subset\left\{(a, \alpha, b) \in A \times H^{s}(\Gamma, U) \times B \mid \pi_{B} \circ \alpha=b\right\}
$$

by the condition that the map

$$
\mathcal{U}_{0} \rightarrow \mathcal{U}:(a, \alpha, b) \mapsto\left(a, \alpha \circ \iota_{a}^{-1}, b\right)
$$

is a bijection. In particular $\alpha\left((\mathrm{i}, 1) \times S^{1}\right) \subset U_{\mathrm{i}, 1}$ and $\alpha\left((\mathrm{i}, 2) \times S^{1}\right) \subset U_{\mathrm{i}, 2}$ for $(a, \alpha, b) \in$ $\mathcal{U}_{0}$. (By a standard construction $H^{s}(\Gamma, U)$ is a complex Hilbert manifold and the subset $\left\{(a, \alpha, b) \mid \pi_{B} \circ \alpha=b\right\}$ is a complex Hilbert submanifold of $A \times H^{s}(\Gamma, U) \times B$. This is because the map $H^{s}(\Gamma, U) \rightarrow H^{s}(\Gamma, B)$ induced by $\pi_{B}$ is a holomorphic submersion. Note that $\mathcal{U}_{0}$ is a connected component of $\left\{(a, \alpha, b) \mid \pi_{B} \circ \alpha=b\right\}$ and hence inherits its Hilbert manifold structure.) We emphasize that the resulting Hilbert manifold structure on $\mathcal{U}$ depends on the choice of the Hardy trivialization. Two different Hardy trivializations give rise to a homeomorphism which is of class $C^{\ell}$ on the dense subset $\mathcal{U} \cap H^{s+\ell}$.

5.7 The fiber isomorphism $f_{0}: P_{a_{0}} \rightarrow Q_{b_{0}}$ determines a point

$$
\left(a_{0}, \alpha_{0}:=f_{0} \mid \Gamma_{a_{0}}, b_{0}\right) \in \mathcal{U}
$$

this point lies in $\mathcal{U}^{\prime} \cap \mathcal{U}^{\prime \prime}$ as

$$
\alpha_{0}=f_{0}^{\prime}\left|\Gamma_{a_{0}}=f_{0}^{\prime \prime}\right| \Gamma_{a_{0}}, \quad \text { where } \quad f_{0}^{\prime}:=f_{0}\left|P_{a_{0}}^{\prime}, \quad f_{0}^{\prime \prime}:=f_{0}\right| P_{a_{0}}^{\prime \prime} .
$$

In the sequel we will denote neighborhoods of $a_{0}$ in $A$ and $\left(a_{0}, \alpha_{0}, b_{0}\right)$ in $\mathcal{U}^{\prime}, \mathcal{U}^{\prime \prime}$, or $\mathcal{U}$ by the same letters $A$, respectively $\mathcal{U}^{\prime}, \mathcal{U}^{\prime \prime}$, or $\mathcal{U}$, and signal this with the text "shrinking $A, \mathcal{U}^{\prime}$, $\mathcal{U}^{\prime \prime}$, or $\mathcal{U}$, if necessary". 
Lemma 5.8 For every $(a, \alpha, b) \in \mathcal{U}^{\prime} \cap \mathcal{U}^{\prime \prime}$ there is a unique fiber isomorphism $f: P_{a} \rightarrow Q_{b}$ with $f \mid \Gamma_{a}=\alpha$.

Proof This follows immediately from [4, Lemma 9.4].

Theorem 5.9 Fix an integer $s+1 / 2>4$. After shrinking $A, \mathcal{U}^{\prime}, \mathcal{U}^{\prime \prime}, \mathcal{U}$, if necessary, the following holds.

(i) For each $a \in A, \mathcal{U}_{a}^{\prime}$ and $\mathcal{U}_{a}^{\prime \prime}$ are complex submanifolds of $\mathcal{U}_{a}$.

(ii) Let $(a, \alpha, b) \in \mathcal{U}^{\prime} \cap \mathcal{U}^{\prime \prime}$ and $f: P_{a} \rightarrow Q_{b}$ be the associated fiber isomorphism with $\alpha=f \mid \Gamma_{a}$. Let $w: \Sigma \rightarrow P_{a}$ be a desingularization with induced structures $j, v, s_{*}, u:=f \circ w$ on $\Sigma$ and $D_{u}$ be the operator in Definition 3.3. Then

$$
\operatorname{ker} D_{u} \cong T_{(\alpha, b)} \mathcal{U}_{a}^{\prime} \cap T_{(\alpha, b)} \mathcal{U}_{a}^{\prime \prime}, \quad \operatorname{coker} D_{u} \cong \frac{T_{(\alpha, b)} \mathcal{U}_{a}}{T_{(\alpha, b)} \mathcal{U}_{a}^{\prime}+T_{(\alpha, b)} \mathcal{U}_{a}^{\prime \prime}}
$$

(iii) $\mathcal{U}^{\prime}$ and $\mathcal{U}^{\prime \prime}$ are complex submanifolds of $\mathcal{U}$.

(iv) The projections $\mathcal{U} \rightarrow A, \mathcal{U}^{\prime} \rightarrow A, \mathcal{U}^{\prime \prime} \rightarrow A$ are holomorphic submersions.

Proof Theorems 9.5 and 11.9 in [4]. The condition $s+1 / 2>4$ is used in compactness arguments for the proofs of (i) and (iii). These compactness arguments can be eliminated by modifying the definition of $\mathcal{U}^{\prime \prime}$ along the lines of the definition of $\mathcal{V}^{\prime \prime}$ in 5.11 below.

5.10 As in [4, Definition 11.6], we use a Hardy trivialization for $\left(\pi_{A}: P \rightarrow A, R_{*}, a_{0}\right)$, i.e. a triple $\left(P^{\prime} \cup P^{\prime \prime}, \iota, \rho\right)$ where $P=P^{\prime} \cup P^{\prime \prime}$ is a Hardy decomposition with corresponding trivialization $\iota: A \times \Gamma \rightarrow \partial P^{\prime}$ as in 5.3 and

$$
\rho: P^{\prime \prime} \rightarrow P_{a_{0}}^{\prime \prime}=: \Omega
$$

is a trivialization such that $\rho_{a}:=\rho \mid P_{a}^{\prime \prime}: P_{a}^{\prime \prime} \rightarrow \Omega$ is a diffeomorphism satisfying

$$
\rho_{a_{0}}=\mathrm{id}, \quad \rho_{a} \circ \iota_{a}=\iota_{a_{0}}
$$

for $a \in A$. We require further that $\rho$ is holomorphic in a neighborhood of the boundary.

5.11 Let $\left(\pi_{A}: P \rightarrow A, R_{*}, a_{0}\right)$ be an unfolding of marked nodal Riemann surfaces and $h_{0}: P_{a_{0}} \rightarrow M$ be a holomorphic map. Choose a Hardy decomposition $P=P^{\prime} \cup P^{\prime \prime}$ as in 5.3 and a Hardy trivialization $\rho_{a}: P_{a} \rightarrow \Omega$ as in 5.10. We would like to imitate Theorem 5.9 and define subsets $\mathcal{V}_{a}^{\prime}, \mathcal{V}_{a}^{\prime \prime} \subset H^{s}\left(\Gamma_{a}, M\right)$ of those maps $\beta \in \mathcal{V}_{a}$ which extend holomorphically to $P_{a}^{\prime}, P_{a}^{\prime \prime}$ respectively, but it is convenient to restrict the extensions. Let

$$
V^{\prime}=V_{1}^{\prime} \cup \cdots \cup V_{\mathrm{k}}^{\prime} \subset M
$$

be an open neighborhood of the image $h_{0}\left(P_{a_{0}} \cap C_{A}\right)$ of the nodal set so that each pair $\left(V_{\mathrm{i}}^{\prime}, h_{0}\left(p_{\mathrm{i}}\right)\right)$ is holomorphically diffeomorphic to the open unit ball in $\mathbb{C}^{\mathrm{m}}$ centered at origin, the closures of the sets $V_{\mathrm{i}}^{\prime}$ are pairwise disjoint, and

$$
h_{0}\left(P_{a_{0}} \cap P_{\mathrm{i}}^{\prime}\right) \subset V_{\mathrm{i}}^{\prime} .
$$

For $a \in A$ abbreviate

$$
\mathcal{V}_{a}:=H^{s}\left(\Gamma_{a}, M\right)
$$

Let $\mathcal{V}_{a}^{\prime} \subset \mathcal{V}_{a}$ be the subspace of those $\beta$ that extend holomorphically to $P_{a}^{\prime}$, i.e.

$$
\mathcal{V}_{a}^{\prime}:=\left\{\beta \in \mathcal{V}_{a} \mid \exists h^{\prime} \in \operatorname{Hol}^{s+1 / 2}\left(P_{a}^{\prime}, M\right) \text { s.t. } h^{\prime}\left(P_{a, \mathrm{i}}^{\prime}\right) \subset V_{\mathrm{i}}^{\prime} \text { and } \beta=h^{\prime} \mid \Gamma_{a}\right\} .
$$


Let $\mathcal{W}_{0}$ be a neighborhood of $h_{0} \mid \Omega$ in $H^{s+1 / 2}(\Omega, M)$, where $\Omega=P_{a_{0}}^{\prime \prime}$ as in 5.10. Via the trivialization $\rho_{a}: P_{a}^{\prime \prime} \rightarrow \Omega$ this determines an open subset

$$
\mathcal{W}_{a}:=\left\{h^{\prime \prime} \in H^{s+1 / 2}\left(P_{a}^{\prime \prime}, M\right) \mid h^{\prime \prime} \circ \rho_{a}^{-1} \in \mathcal{W}_{0}\right\}
$$

of $H^{s+1 / 2}\left(P_{a}^{\prime \prime}, M\right)$ for $a \in A$. Let

$$
\mathcal{V}_{a}^{\prime \prime}:=\left\{\beta \in \mathcal{V}_{a} \mid \exists h^{\prime \prime} \in \mathcal{W}_{a} \cap \mathrm{Hol}^{s+1 / 2}\left(P_{a}^{\prime \prime}, M\right) \text { s.t. } \beta=h^{\prime \prime} \mid \Gamma_{a}\right\}
$$

Define

$$
\mathcal{V}:=\bigsqcup_{a \in A} \mathcal{V}_{a}, \quad \mathcal{V}^{\prime}:=\bigsqcup_{a \in A} \mathcal{V}_{a}^{\prime}, \quad \mathcal{V}^{\prime \prime}:=\bigsqcup_{a \in A} \mathcal{V}_{a}^{\prime \prime}
$$

Then every pair $(a, \beta) \in \mathcal{V}^{\prime} \cap \mathcal{V}^{\prime \prime}$ determines a holomorphic map $h: P_{a} \rightarrow M$ such that $h \mid \Gamma_{a}=\beta$. As in 5.6 we use the nodal coordinate system of 5.3 to construct an auxiliary Hilbert manifold structure on $\mathcal{V}$ via the bijection

$$
\mathcal{V} \rightarrow A \times H^{s}(\Gamma, M):(a, \beta) \mapsto\left(a, \beta \circ \iota_{a}\right) .
$$

Theorem 5.12 Continue the notation of 5.3, 5.10, and 5.11. Fix an integer $s+1 / 2>1$. After shrinking $A$ and $\mathcal{W}_{0}$, if necessary, the following holds.

(i) For each $a \in A, \mathcal{V}_{a}^{\prime}$ and $\mathcal{V}_{a}^{\prime \prime}$ are complex submanifolds of $\mathcal{V}_{a}$

(ii) Let $(a, \beta) \in \mathcal{V}^{\prime} \cap \mathcal{V}^{\prime \prime}$ and $h: P_{a} \rightarrow M$ be the associated holomorphic map with $\beta=h \mid \Gamma_{a}$. Let $w: \Sigma \rightarrow P_{a}$ be a desingularization with induced structures $s_{*}, v, j$, $v:=h \circ w$ on $\Sigma$ and $D_{v}$ be the operator in 3.3. Then

$$
\operatorname{ker} D_{v} \cong T_{\beta} \mathcal{V}_{a}^{\prime} \cap T_{\beta} \mathcal{V}_{a}^{\prime \prime}, \quad \operatorname{coker} D_{v} \cong \frac{T_{\beta} \mathcal{V}_{a}}{T_{\beta} \mathcal{V}_{a}^{\prime}+T_{\beta} \mathcal{V}_{a}^{\prime \prime}}
$$

(iii) $\mathcal{V}^{\prime}$ and $\mathcal{V}^{\prime \prime}$ are complex submanifolds of $\mathcal{V}$

(iv) The projections $\mathcal{V} \rightarrow A, \mathcal{V}^{\prime} \rightarrow A, \mathcal{V}^{\prime \prime} \rightarrow A$ are holomorphic submersions.

Proof of Theorem 5.12 (i) and (ii) In parts (i) and (ii) the point $a$ is fixed. We introduce the following notation to make the proof look more like the proof of [4, Theorem 9.5]. Use the notation of part (ii). Abbreviate

$$
\Sigma^{\prime}:=w^{-1}\left(P_{a}^{\prime}\right), \quad \Sigma^{\prime \prime}:=w^{-1}\left(P_{a}^{\prime \prime}\right) .
$$

Thus $\Sigma^{\prime}$ and $\Sigma^{\prime \prime}$ are submanifolds of $\Sigma$ such that

$$
\Sigma=\Sigma^{\prime} \cup \Sigma^{\prime \prime}, \quad \partial \Sigma^{\prime}=\partial \Sigma^{\prime \prime}=\Sigma^{\prime} \cap \Sigma^{\prime \prime} .
$$

Now $w^{-1} \circ \iota_{a}$ is a diffeomorphism from $\Gamma$ in (26) to $\Sigma^{\prime} \cap \Sigma^{\prime \prime}$. To simplify the notation we assume that $\Gamma=\Sigma^{\prime} \cap \Sigma^{\prime \prime}$. The submanifold $\Sigma^{\prime}$ is a disjoint union

$$
\Sigma^{\prime}=\Sigma_{1}^{\prime} \cup \cdots \cup \Sigma_{\mathrm{k}}^{\prime}
$$

where each set $\Sigma_{\mathrm{i}}^{\prime}$ is either an embedded closed annulus or else the union of two disjoint embedded closed disks centered at two equivalent nodal points. It follows that every pair of equivalent nodal points appears in some $\Sigma_{\mathrm{i}}^{\prime}$. In case $\Sigma_{\mathrm{i}}^{\prime}$ is a disjoint union of two disks, say $\Sigma_{\mathrm{i}}^{\prime}=\Sigma_{\mathrm{i}, 1}^{\prime} \cup \Sigma_{\mathrm{i}, 2}^{\prime}$, choose holomorphic diffeomorphisms $x_{\mathrm{i}}: \Sigma_{\mathrm{i}, 1}^{\prime} \rightarrow \mathbb{D}$ and $y_{\mathrm{i}}: \Sigma_{\mathrm{i}, 2}^{\prime} \rightarrow \mathbb{D}$ which send the nodal point to 0 . In case $\Sigma_{\mathrm{i}}^{\prime}$ is an annulus choose a holomorphic diffeomorphism $x_{\mathrm{i}}: \Sigma_{\mathrm{i}}^{\prime} \rightarrow \mathbb{A}\left(\delta_{\mathrm{i}}, 1\right)$ and define $y_{\mathrm{i}}: \Sigma_{\mathrm{i}}^{\prime} \rightarrow \mathbb{A}\left(\delta_{\mathrm{i}}, 1\right)$ by $y_{\mathrm{i}}=\delta_{\mathrm{i}} / x_{\mathrm{i}}$.

Let $\mathcal{V}_{0}^{\prime} \subset H^{s}(\Gamma, M)$ be the subspace of those $H^{s}$-functions $\gamma: \Gamma \rightarrow M$ that extend holomorphically to $H^{s+1 / 2}$-functions $v^{\prime}: \Sigma^{\prime} \rightarrow M$ which map each pair of equivalent nodal 
points to the same point in $M$ and take $\Sigma_{\mathrm{i}}^{\prime}$ to $V_{\mathrm{i}}^{\prime}$. Let $\mathcal{V}_{0}^{\prime \prime} \subset H^{s}(\Gamma, M)$ be the subspace of those $H^{s}$-functions $\gamma: \Gamma \rightarrow M$ that extend holomorphically to $H^{s+1 / 2}$-functions $v^{\prime \prime}: \Sigma^{\prime \prime} \rightarrow M$ such that $h^{\prime \prime}:=v^{\prime \prime} \circ w^{-1} \mid P_{a}^{\prime \prime} \in \mathcal{W}_{a}$. In this notation part (i) asserts that $\mathcal{V}_{0}^{\prime}$ and $\mathcal{V}_{0}^{\prime \prime}$ are complex submanifolds of $H^{s}(\Gamma, M)$.

We prove that $\mathcal{V}_{0}^{\prime}$ is a complex submanifold of $H^{s}(\Gamma, M)$. Choose coordinate charts $\psi_{\mathrm{i}}: V_{\mathrm{i}}^{\prime} \rightarrow \mathbb{C}^{\mathrm{m}}$ such that $\psi_{\mathrm{i}}\left(H_{A}\left(p_{\mathrm{i}}\right)\right)=0$ and $\psi_{\mathrm{i}}\left(V_{\mathrm{i}}^{\prime}\right)$ is the open unit ball in $\mathbb{C}^{\mathrm{m}}$ for every $\mathrm{i}$. Define the map

$$
\mathcal{V}_{0}^{\prime} \rightarrow\left(H^{s}\left(S^{1}, \mathbb{C}^{\mathrm{m}}\right)\right)^{2 \mathrm{k}}: \gamma \mapsto\left(\xi_{1}, \eta_{1}, \ldots, \xi_{\mathrm{k}}, \eta_{\mathrm{k}}\right)
$$

by

$$
\xi_{\mathrm{i}}:=\psi_{\mathrm{i}} \circ \gamma \circ x_{\mathrm{i}}^{-1}, \quad \eta_{\mathrm{i}}:=\psi_{\mathrm{i}} \circ \gamma \circ y_{\mathrm{i}}^{-1} .
$$

The image of (28) is the set of all tuples $\left(\xi_{1}, \eta_{1}, \ldots, \xi_{\mathrm{k}}, \eta_{\mathrm{k}}\right)$ in $\left(H^{s}\left(S^{1}, \mathbb{C}^{\mathrm{m}}\right)\right)^{2 \mathrm{k}}$ that satisfy the following conditions.

(a) The functions $\xi_{\mathrm{i}}, \eta_{\mathrm{i}}: S^{1} \rightarrow \mathbb{C}^{\mathrm{m}}$ take values in the open unit ball.

(b) If $\Sigma_{\mathrm{i}}^{\prime}$ is the disjoint union of two discs then all negative Fourier coefficients of $\xi_{\mathrm{i}}$ and $\eta_{\mathrm{i}}$ vanish and the zeroth coefficients agree.

(c) If $\Sigma_{\mathrm{i}}^{\prime}$ is an annulus then $\gamma_{\mathrm{i}, 1}$ extends holomorphically to an $H^{s+1 / 2}$ function on the annulus $\mathbb{A}\left(\delta_{\mathrm{i}}, 1\right)$ and $\eta_{\mathrm{i}}(y)=\xi_{\mathrm{i}}\left(\delta_{\mathrm{i}} / y\right)$ for every $y \in S^{1}$.

Conditions (b) and (c) define a closed subspace of $\left(H^{s}\left(S^{1}, \mathbb{C}^{\mathrm{m}}\right)\right)^{2 \mathrm{k}}$ and condition (a) defines an open set in this subspace. Hence the image of (28) is an open set in a Hilbert subspace and this shows that $\mathcal{V}_{0}^{\prime}$ is a Hilbert submanifold of $H^{s}(\Gamma, M)$.

We prove that $\mathrm{Hol}^{s+1 / 2}\left(\Sigma^{\prime \prime}, M\right)$ is a complex submanifold of $H^{s+1 / 2}\left(\Sigma^{\prime \prime}, M\right)$. To see this note that the Cauchy-Riemann operator $v^{\prime \prime} \mapsto \bar{\partial}_{j, J}\left(v^{\prime \prime}\right)$ defines a holomorphic section of the vector bundle $\mathcal{E} \rightarrow \mathcal{B}:=H^{s+1 / 2}\left(\Sigma^{\prime \prime}, M\right)$ with fibers

$$
\mathcal{E}_{v^{\prime \prime}}:=H^{s-1 / 2}\left(\Sigma^{\prime \prime}, \Lambda^{0,1} T^{*} \Sigma^{\prime \prime} \otimes\left(v^{\prime \prime}\right)^{*} T M\right)
$$

The intrinsic derivative of this section at a zero $v^{\prime \prime}$ is the Cauchy-Riemann operator $D_{v^{\prime \prime}}$ : $T_{v^{\prime \prime}} \mathcal{B} \rightarrow \mathcal{E}_{v^{\prime \prime}}$ of the holomorphic vector bundle $\left(v^{\prime \prime}\right)^{*} T M \rightarrow \Sigma^{\prime \prime}$. Since each component of $\Sigma^{\prime \prime}$ has nonempty boundary the operator $D_{v^{\prime \prime}}$ is surjective; a right inverse can be constructed from an appropriate Lagrangian boundary condition (see [2, Appendix C.1.10]). This proves that $\mathrm{Hol}^{s+1 / 2}\left(\Sigma^{\prime \prime}, M\right)$ is a complex submanifold of $H^{s+1 / 2}\left(\Sigma^{\prime \prime}, M\right)$.

We prove that $\mathcal{V}_{0}^{\prime \prime}$ is a complex submanifold of $H^{s}(\Gamma, M)$. The restriction map

$$
\operatorname{Hol}^{s+1 / 2}\left(\Sigma^{\prime \prime}, M\right) \rightarrow \mathcal{V}_{0}: v^{\prime \prime} \mapsto v^{\prime \prime} \mid \Gamma
$$

is an injective holomorphic immersion. That it is holomorphic is obvious, that it is injective follows from unique continuation, and that it is an immersion follows from the elliptic boundary estimate in [4, Theorem B.4]. It follows that the image of a sufficiently small neighborhood of $H_{A} \circ w \mid \Sigma^{\prime \prime}$ under the restriction map is a complex submanifold of $H^{s}(\Gamma, M)$; this image is $\mathcal{V}_{0}^{\prime \prime}$. This proves (i).

We prove (ii). It follows directly from the definitions that there is a map

$$
\operatorname{ker} D_{v} \rightarrow T_{\beta} \mathcal{V}_{a}^{\prime} \cap T_{\beta} \mathcal{V}_{a}^{\prime \prime}: \hat{v} \mapsto \hat{v} \circ w^{-1} \mid \Gamma_{a} .
$$

As in the proof of Theorem 9.5 (ii) in [4] this map is injective by unique continuation and is surjective by elliptic regularity. Now define a map

$$
\operatorname{coker} D_{v} \rightarrow \frac{T_{\beta} \mathcal{V}_{a}}{T_{\beta} \mathcal{V}_{a}^{\prime}+T_{\beta} \mathcal{V}_{a}^{\prime \prime}}:[\eta] \mapsto[\hat{\beta}]
$$


as follows. Given $\eta \in \Omega^{0,1}\left(\Sigma, v^{*} T M\right)$ choose two vector fields $\xi^{\prime}$ along $v^{\prime}:=v \mid \Sigma^{\prime}$ and $\xi^{\prime \prime}$ along $v^{\prime \prime}:=v \mid \Sigma^{\prime \prime}$ that satisfy

$$
D_{v^{\prime}} \xi^{\prime}=\eta\left|\Sigma^{\prime}, \quad D_{v^{\prime \prime}} \xi^{\prime \prime}=\eta\right| \Sigma^{\prime \prime}, \quad \xi^{\prime}\left|\Gamma-\xi^{\prime \prime}\right| \Gamma=\hat{\beta} \circ w \mid \Gamma .
$$

One verifies as in the proof of [4, Theorem 9.5 (iii)] that this map is well defined and bijective. That this map is well defined follows directly from the definitions and that it is injective uses elliptic regularity. The proof of surjectivity is based on the following two assertions.

(a) Each element in the quotient $T_{\beta} \mathcal{V}_{a} /\left(T_{\beta} \mathcal{V}_{a}^{\prime}+T_{\beta} \mathcal{V}_{a}^{\prime \prime}\right)$ can be represented by a smooth vector field along $\beta$.

(b) For every smooth vector field $\hat{\beta}$ along $\beta$ there exist vector fields $\xi^{\prime}$ along $v^{\prime}$ and $\xi^{\prime \prime}$ along $v^{\prime \prime}$ such that $\xi^{\prime}\left|\Gamma-\xi^{\prime \prime}\right| \Gamma=\hat{\beta} \circ w \mid \Gamma$ and the $(0,1)$-form $\eta$ along $v$ defined by $\eta \mid \Sigma^{\prime}:=D_{v^{\prime}} \xi^{\prime}$ and $\eta \mid \Sigma^{\prime \prime}:=D_{v^{\prime \prime}} \xi^{\prime \prime}$ is smooth.

One first proves (b) by an argument in local coordinates, using the construction due to Emile Borel of a smooth function with a prescribed Taylor series at a point. Once (b) is established assertion (a) follows from the observation that the subspace of those elements of the quotient $T_{\beta} \mathcal{V}_{a} /\left(T_{\beta} \mathcal{V}_{a}^{\prime}+T_{\beta} \mathcal{V}_{a}^{\prime \prime}\right)$ that admit smooth representatives is both finite dimensional and dense. The details are exactly as in the proof of [4, Theorem 9.5 (iii)] and will be omitted. Thus we have proved (ii). The proofs of (iii) and (iv) are given below after some preparation.

5.13 Let $\mathbb{D} \subset \mathbb{C}$ be the closed unit disc. The standard node is defined as the map

$$
N \rightarrow \operatorname{int}(\mathbb{D}):(x, y) \mapsto x y, \quad N:=\{(x, y) \in \mathbb{D} \times \mathbb{D}|| x y \mid<1\} .
$$

For $z \in \operatorname{int}(\mathbb{D})$ denote

$$
N_{z}:=\{(x, y) \in \mathbb{D} \times \mathbb{D} \mid x y=z\} .
$$

The boundary $\partial N_{z}$ has two components

$$
\partial_{1} N_{z}:=\left\{(x, y) \in N_{z}|| x \mid=1\right\}, \quad \partial_{2} N_{z}:=\left\{(x, y) \in N_{z}|| y \mid=1\right\}
$$

which can be identified with the unit circle $S^{1}=\partial \mathbb{D} \subset \mathbb{C}$ via the embeddings $\iota_{1}, \iota_{2}: S^{1} \rightarrow N_{z}$ given by

$$
\iota_{1, z}\left(e^{i \theta}\right):=\left(e^{i \theta}, e^{-i \theta} z\right), \quad \iota_{2, z}\left(e^{i \theta}\right):=\left(e^{-i \theta} z, e^{i \theta}\right) .
$$

We study the set of all triples $(z, \xi, \eta)$ where $z \in \operatorname{int}(\mathbb{D})$ and $\xi: S^{1} \rightarrow \mathbb{C}^{\mathrm{m}}, \eta: S^{1} \rightarrow \mathbb{C}^{\mathrm{m}}$ are the boundary values a holomorphic map $v: N_{z} \rightarrow \mathbb{C}^{\mathrm{m}}$, namely

$$
\xi:=v \circ \iota_{1, z}, \quad \eta:=v \circ \iota_{2, z} .
$$

At $z=0$, the functions $\xi$ and $\eta$ extend to the closed unit disk and agree at the origin. More precisely, fix an integer $s+1 / 2>1$. For $z \in \operatorname{int}(\mathbb{D}) \backslash 0$ let $\mathrm{Hol}^{s+1 / 2}\left(N_{z}, \mathbb{C}^{\mathrm{m}}\right)$ be the space of all maps $v: N_{z} \rightarrow \mathbb{C}^{\mathrm{m}}$ of class $H^{s+1 / 2}$ which are holomorphic in $\operatorname{int}\left(N_{z}\right)$. The space $N_{0}$ consists of two disks $\mathbb{D} \times 0$ and $0 \times \mathbb{D}$ intersecting in $(0,0)$. In this case let $\operatorname{Hol}^{s+1 / 2}\left(N_{0}, \mathbb{C}^{\mathrm{m}}\right)$ denote the space of all continuous maps $v: N_{0} \rightarrow \mathbb{C}^{\mathrm{m}}$ such that $v_{1}:=v \mid \mathbb{D} \times 0$ and $v_{2}:=v \mid 0 \times \mathbb{D}$ are holomorphic in the interior and restrict to $H^{s}$ functions on the boundary. In both cases the trace theorem gives rise to a map

$$
\operatorname{Hol}^{s+1 / 2}\left(N_{z}, \mathbb{C}^{\mathrm{m}}\right) \rightarrow H^{s}\left(S^{1}, \mathbb{C}^{\mathrm{m}}\right) \times H^{s}\left(S^{1}, \mathbb{C}^{\mathrm{m}}\right) ; v \mapsto\left(v \circ \iota_{1, z}, v \circ \iota_{2, z}\right) .
$$

The norm on $H^{s}\left(S^{1}, \mathbb{C}^{\mathrm{m}}\right)$ is given by

$$
\|\zeta\|_{s}:=\sqrt{\sum_{n \in \mathbb{Z}}(1+|n|)^{2 s}\left|\zeta_{n}\right|^{2}}, \quad \zeta\left(e^{i \theta}\right)=\sum_{n \in \mathbb{Z}} \zeta_{n} e^{i n \theta} .
$$




\section{Lemma 5.14 (i) The set}

$$
\mathcal{N}:=\left\{(z, \xi, \eta) \mid \exists v \in \operatorname{Hol}^{s+1 / 2}\left(N_{z}, \mathbb{C}^{\mathrm{m}}\right) \text { s.t. } \xi=v \circ \iota_{1, z}, \eta=v \circ \iota_{2, z}\right\} .
$$

is a complex submanifold of $H^{s}\left(S^{1}, \mathbb{C}^{\mathrm{m}}\right) \times H^{s}\left(S^{1}, \mathbb{C}^{\mathrm{m}}\right) \times \operatorname{int}(\mathbb{D})$.

(ii) The projection $\mathcal{N} \rightarrow \operatorname{int}(\mathbb{D}):(\xi, \eta, z) \mapsto z$ is a surjective submersion.

(iii) Let $A \subset \operatorname{int}(\mathbb{D}) \times \mathbb{C}^{\mathrm{a}-1}$ be an open set and $A \rightarrow \mathcal{N}:(z, t) \mapsto\left(z, \xi_{z, t}, \eta_{z, t}\right)$ be a holomorphic map. Then the map

$$
H:\left\{(x, y, t) \in \mathbb{C}^{\mathrm{a}+1} \mid x, y \in \operatorname{int}(\mathbb{D}),(x y, t) \in A\right\} \rightarrow \mathbb{C}^{\mathrm{m}}
$$

well defined by

$$
H(x, y, t):= \begin{cases}\xi_{x y, t}(x), & \text { if } y \neq 0 \\ \eta_{x y, t}(y), & \text { if } x \neq 0 \\ \xi_{0, t}(0)=\eta_{0, t}(0), & \text { if } x=y=0\end{cases}
$$

is holomorphic.

Proof Let $(z, \xi, \eta) \in \operatorname{int}(\mathbb{D}) \times H^{s}\left(S^{1}, \mathbb{C}^{\mathrm{m}}\right) \times H^{s}\left(S^{1}, \mathbb{C}^{\mathrm{m}}\right)$ and write

$$
\xi(x)=: \sum_{n \in \mathbb{Z}} \xi_{n} x^{n}, \quad \eta(y)=: \sum_{n \in \mathbb{Z}} \eta_{n} y^{n},
$$

i.e. $\xi_{n}, \eta_{n} \in \mathbb{C}^{\mathrm{m}}$ are the Fourier coefficients of $\xi, \eta$. When $(z, \xi, \eta) \in \mathcal{N}$ each of these series converges on the annulus with inner radius $|z|$ and outer radius one. (Thus was used in defining $H$.) When $z \neq 0$ we have

$$
(z, \xi, \eta) \in \mathcal{N} \Longleftrightarrow \eta_{-n}=z^{n} \xi_{n} \quad \text { for all } n \in \mathbb{Z},
$$

but

$$
(0, \xi, \eta) \in \mathcal{N} \Longleftrightarrow \xi_{0}=\eta_{0}, \quad \xi_{n}=\eta_{n}=0 \quad \text { for } n<0 .
$$

Denote by $H_{ \pm}^{s}\left(S^{1}, \mathbb{C}^{\mathrm{m}}\right) \subset H^{s}\left(S^{1}, \mathbb{C}^{\mathrm{m}}\right)$ the Hardy space of all $\zeta \in H^{s}\left(S^{1}, \mathbb{C}^{\mathrm{m}}\right)$ whose Fourier coefficients $\zeta_{n}$ vanish for $\mp n \geq 0$. For $z \in \operatorname{int}(\mathbb{D})$ define the bounded linear operator $\mathcal{T}_{z}: H_{+}^{s}\left(S^{1}, \mathbb{C}^{\mathrm{m}}\right) \rightarrow H_{-}^{s}\left(S^{1}, \mathbb{C}^{\mathrm{m}}\right)$ by

$$
\mathcal{T}_{z}\left(\sum_{n>0} c_{n} e^{i n \theta}\right):=\sum_{n>0} z^{n} c_{n} e^{-i n \theta} .
$$

Then the resulting map

$$
\operatorname{int}(\mathbb{D}) \times H_{+}^{s}\left(S^{1}, \mathbb{C}^{\mathrm{m}}\right) \rightarrow H_{-}^{s}\left(S^{1}, \mathbb{C}^{\mathrm{m}}\right):\left(z, \zeta_{+}\right) \mapsto \mathcal{T}_{z}\left(\zeta_{+}\right)
$$

is holomorphic. Moreover, the set $\mathcal{N}$ can be written in the form

$$
\mathcal{N}=\left\{\begin{array}{l|l}
\left(z, \xi_{+}+\lambda+\mathcal{T}_{z}\left(\eta_{+}\right), \eta_{+}+\lambda+\mathcal{T}_{z}\left(\xi_{+}\right)\right) & \begin{array}{l}
\xi_{+}, \eta_{+} \in H_{+}^{s}\left(S^{1}, \mathbb{C}^{\mathrm{m}}\right), \\
\lambda \in \mathbb{C}^{\mathrm{m}}, z \in \operatorname{int}(\mathbb{D})
\end{array}
\end{array}\right\} .
$$

Hence $\mathcal{N}$ is a complex Hilbert submanifold of the space

$$
\mathbb{C} \times H^{s}\left(S^{1}, \mathbb{C}^{\mathrm{m}}\right)^{2} \cong \mathbb{C} \times H_{+}^{s}\left(S^{1}, \mathbb{C}^{\mathrm{m}}\right)^{2} \times\left(\mathbb{C}^{\mathrm{m}}\right)^{2} \times H_{-}^{s}\left(S^{1}, \mathbb{C}^{\mathrm{m}}\right)^{2} .
$$

The formula shows that the projection $\mathcal{N} \rightarrow \operatorname{int}(\mathbb{D})$ is a surjective submersion. This proves (i) and (ii). 
To prove (iii) we observe that the projection $H^{s}\left(S^{1}, \mathbb{C}^{\mathrm{m}}\right) \rightarrow H_{+}^{s}\left(S^{1}, \mathbb{C}^{\mathrm{m}}\right)$ and the evaluation map int $(\mathbb{D}) \times H_{+}^{s}\left(S^{1}, \mathbb{C}^{m}\right) \rightarrow \mathbb{C}^{\mathrm{m}}:(z, \zeta) \mapsto \zeta(z)$ are holomorphic. Hence (iii) follows from the identification

$$
H(x, y, t)=\xi_{x y, t,+}(x)+\eta_{x y, t,+}(y)+\lambda(x y, t)
$$

where $\lambda(z, t)$ denotes the common constant term of the power series $\xi_{z, t}$ and $\eta_{z, t}$. This proves the lemma.

Proof of Theorem 5.12 (iii) and (iv) We prove that $\mathcal{V}^{\prime}$ is a complex Hilbert submanifold of $\mathcal{V}$. As in the proof of (i) we choose holomorphic coordinate charts $\psi_{\mathrm{i}}: V_{\mathrm{i}}^{\prime} \rightarrow \mathbb{C}^{\mathrm{m}}$ such that $\psi_{\mathrm{i}}\left(p_{\mathrm{i}}\right)=0$ and $\psi_{\mathrm{i}}\left(V_{\mathrm{i}}^{\prime}\right)$ is the open unit disc in $\mathbb{C}^{\mathrm{m}}$ for every $\mathrm{i}$. Define the map

$$
\mathcal{V}^{\prime} \rightarrow A \times\left(H^{s}\left(S^{1}, \mathbb{C}^{\mathrm{m}}\right)\right)^{2 \mathrm{k}}:(a, \beta) \mapsto\left(a, \xi_{1}, \eta_{1}, \ldots, \xi_{\mathrm{k}}, \eta_{\mathrm{k}}\right)
$$

by

$$
\xi_{\mathrm{i}}:=\psi_{\mathrm{i}} \circ \beta \circ x_{\mathrm{i}}^{-1}, \quad \eta_{\mathrm{i}}:=\psi_{\mathrm{i}} \circ \beta \circ y_{\mathrm{i}}^{-1} .
$$

as in (29). The image of this map is the subset

$$
\left.\left\{\left(a, \xi_{1}, \eta_{1}, \ldots, \xi_{\mathrm{k}}, \eta_{\mathrm{k}}\right) \in A \times H^{s}\left(S^{1}, \mathbb{C}^{\mathrm{m}}\right)\right)^{2 \mathrm{k}} \mid\left(z_{\mathrm{i}}(a), \xi_{\mathrm{i}}, \eta_{\mathrm{i}}\right) \in \mathcal{N} \forall \mathrm{i}\right\} .
$$

By Lemma 5.14, this set is a complex Hilbert submanifold of $A \times\left(H^{s}\left(S^{1}, \mathbb{C}^{\mathrm{m}}\right)\right)^{2 \mathrm{k}}$. Hence $\mathcal{V}^{\prime}$ is a complex Hilbert submanifold of $\mathcal{V}^{\prime}$ and the projection $\mathcal{V} \rightarrow A$ is a submersion.

The proof that $\mathcal{V}^{\prime \prime}$ is a complex Hilbert submanifold of $\mathcal{V}$ follows the argument in the proof of $[4$, Theorem 11.9 (ii)]. Define

$$
\begin{aligned}
& \mathcal{B}:=\left\{\left(a, h^{\prime \prime}\right) \mid a \in A, h^{\prime \prime} \in H^{s+1 / 2}\left(P_{a}^{\prime \prime}, M\right)\right\}, \\
& \mathcal{Z}:=\left\{\left(a, h^{\prime \prime}\right) \in \mathcal{B} \mid h^{\prime \prime} \in \operatorname{Hol}^{s+1 / 2}\left(P_{a}^{\prime \prime}, M\right)\right\} .
\end{aligned}
$$

We construct an auxiliary Hilbert manifold structure on $\mathcal{B}$ and show that $\mathcal{Z}$ is a smooth submanifold of $\mathcal{B}$. Fix a Hardy trivialization $\left(P=P^{\prime} \cup P^{\prime \prime}, \iota, \rho\right)$ as in 5.10 and denote

$$
\mathcal{B}_{0}:=\left\{(a, w) \mid a \in A, w \in H^{s+1 / 2}(\Omega, M)\right\}
$$

This space is a Hilbert manifold and the Hardy trivialization induces a bijection

$$
\mathcal{B}_{0} \rightarrow \mathcal{B}:(a, w) \mapsto\left(a, h^{\prime \prime}:=w \circ \rho_{a}\right) .
$$

This defines the Hilbert manifold structure on $\mathcal{B}$. The bijection $\mathcal{B}_{0} \rightarrow \mathcal{B}$ identifies the subset $\mathcal{Z} \subset \mathcal{B}$ with the subset $\mathcal{Z}_{0} \subset \mathcal{B}_{0}$ given by

$$
\mathcal{Z}_{0}:=\left\{(a, w) \in \mathcal{B}_{0} \mid w \in \operatorname{Hol}^{s+1 / 2}((\Omega, j(a)), M)\right\},
$$

where $j(a):=\left(\rho_{a}\right)_{*}\left(J_{P} \mid P_{a}^{\prime \prime}\right), \rho_{a}: P_{a}^{\prime \prime} \rightarrow \Omega$ is the Hardy trivialization, and $J_{P}$ is the complex structure on $P$. (The map $a \mapsto j(a)$ need not be holomorphic).

We prove that $\mathcal{Z}_{0}$ is a smooth Hilbert submanifold of $\mathcal{B}_{0}$. The tangent space of $\mathcal{B}_{0}$ at a pair $(a, w)$ is

$$
T_{a, w} \mathcal{B}_{0}=T_{a} A \times H^{s+1 / 2}\left(\Omega, w^{*} T M\right) .
$$

Let $\mathcal{E} \rightarrow \mathcal{B}_{0}$ be the complex Hilbert space bundle whose fiber

$$
\mathcal{E}_{a, w}:=H^{s-1 / 2}\left(\Omega, \Lambda_{j(a)}^{0,1} T^{*} \Omega \otimes w^{*} T M\right)
$$


over $(a, w) \in \mathcal{B}_{0}$ is the Sobolev space of $(0,1)$-forms on $(\Omega, j(a))$ of class $H^{s-1 / 2}$ with values in the pullback tangent bundle $w^{*} T M$. As before the Cauchy-Riemann operator defines a smooth section $\bar{\partial}: \mathcal{B}_{0} \rightarrow \mathcal{E}$ given by

$$
\bar{\partial}(a, w):=\bar{\partial}_{j(a), J}(w)=\frac{1}{2}(d w+J \circ d w \circ j(a)) .
$$

Here $J$ denotes the complex structure on $M$. The zero set of this section is the set $\mathcal{Z}_{0}$ defined above. It follows as in the proof of (i) that the linearized operator $D_{a, w}: T_{a, v^{\prime \prime}} \mathcal{B}_{0} \rightarrow \mathcal{E}_{a, w}$ is surjective and has a right inverse. Hence the zero set $\mathcal{Z}_{0}$ is a smooth Hilbert submanifold of $\mathcal{B}_{0}$. Again as in the proof of (i) restriction to the boundary gives rise to a smooth injective immersion

$$
\mathcal{Z}_{0} \rightarrow \mathcal{V}:(a, w) \mapsto(a, \beta), \quad \beta:=w \circ \rho_{a}^{-1} \mid \Gamma_{a} .
$$

The image of a sufficiently small neighbourhood of $\left(a_{0}, w_{0}:=H_{A} \mid \Omega\right)$ under this immersion is $\mathcal{V}^{\prime \prime}$; the neighborhood is $\mathcal{Z}_{0} \cap\left(A \times \mathcal{W}_{0}\right)$ after shrinking $A$ and $\mathcal{W}_{0}$, if necessary. Hence $\mathcal{V}^{\prime \prime}$ is a smooth Hilbert submanifold of $\mathcal{V}$. That it is a complex submanifold follows, as in the proof of Theorem 11.9 in [4], by introducing an auxiliary (almost) complex structure on $\mathcal{Z}_{0}$. Namely, the push forward of the complex structure on $P^{\prime \prime}$ by the Hardy trivialization

$$
\pi_{A} \times \rho: P^{\prime \prime} \rightarrow A \times \Omega
$$

of 5.10 has the form (14) for a smooth map $j: A \rightarrow \mathcal{J}(\Omega)$ and a smooth 1-form $\eta: T A \rightarrow$ $\operatorname{Vect}(\Omega)$ satisfying (13) and (17). Since $\rho$ is holomorphic near $\partial P^{\prime}$ with respect to the complex structure of $\Omega$ it follows that $\eta$ vanishes near $A \times \partial \Omega$. The tangent space $T_{(a, w)} \mathcal{Z}_{0}$ is the kernel of the operator $\mathcal{D}_{a, w}$ from $T_{(a, w)} \mathcal{B}_{0}$ to $\Omega_{j(a)}^{0,1}\left(\Omega, w^{*} T M\right)$ given by

$$
\mathcal{D}_{(a, w)}(\hat{a}, \hat{w})=D_{w} \hat{w}+\frac{1}{2} J(w) d w \cdot d j(a) \hat{a} .
$$

It follows from (13) and (17) that the automorphisms

$$
(\hat{a}, \hat{w}) \mapsto(\sqrt{-1} \hat{a}, J(w) \hat{w}-d w \cdot \eta(a, \hat{a}))
$$

define an almost complex structure on $\mathcal{Z}_{0}$. Since $\eta$ vanishes near the boundary, the embedding

$$
\mathcal{Z}_{0} \rightarrow A \times H^{s}(\Gamma, M):(a, w) \mapsto\left(a, w \circ \iota_{a_{0}}\right)
$$

is holomorphic. Hence $\mathcal{V}^{\prime \prime}$ is a complex submanifold of $\mathcal{V}$ as claimed.

That the projection $\mathcal{V}^{\prime \prime} \rightarrow A$ is a submersion follows from the fact that the linearized operator (31) of the Sect. (30) is already surjective when differentiating in the direction of a vector field $\hat{v}$ along $v$. This completes the proof Theorem 5.12.

Definition 5.15 Let $\pi_{A}: P \rightarrow A$ be a nodal family and denote by

$$
C_{1}, \ldots, C_{\mathrm{k}} \subset P
$$

the components of the singular set near $P_{a_{0}}$. The set

$$
A_{0}:=\pi_{A}\left(C_{1}\right) \cap \cdots \cap \pi_{A}\left(C_{\mathrm{k}}\right)
$$

is called the core of the family. Recall from [4, Definition 12.1] that we call $\pi_{A}$ regular nodal if the submanifolds $\pi_{A}\left(C_{\mathrm{i}}\right)$ intersect transversally. In this case, the core $A_{0}$ is a complex submanifold of $A$ of codimension $\mathrm{k}$. We call an unfolding $\left(\pi_{A}: P \rightarrow A, R_{*}, a_{0}\right)$ regular nodal iff the ambient family $\pi_{A}: P \rightarrow A$ is regular nodal. In [4, Theorem 5.6] we constructed 
a universal unfolding which is regular nodal. By the uniqueness of universal unfoldings it follows (after shrinking $A$ if necessary) that every universal unfolding is regular nodal.

Theorem 5.16 Continue the notation of 5.3, 5.10, 5.11, and Definition 5.15, and fix an integer $s+1 / 2>1$. Assume that the unfolding $\left(\pi_{A}, R_{*}, a_{0}\right)$ (of marked nodal Riemann surfaces) is universal. Let $w_{0}: \Sigma \rightarrow P_{a_{0}}$ be a desingularization with induced structures $s_{0, *}, v_{0}, j_{0}$, $v_{0}:=h_{0} \circ w_{0}$ on $\Sigma$. Then the configuration $\left(\Sigma, s_{0, *}, v_{0}, j_{0}, v_{0}\right)$ is stable; assume that it is regular. Then the following holds.

(i) $\mathcal{V}^{\prime}$ and $\mathcal{V}^{\prime \prime}$ intersect transversally in $\mathcal{V}$ at $\left(a_{0}, \beta_{0}:=h_{0} \mid \Gamma_{a_{0}}\right)$.

(ii) The projection $\mathcal{V}^{\prime} \cap \mathcal{V}^{\prime \prime} \rightarrow A$ is tranverse to $A_{0}$ at $\left(a_{0}, \beta_{0}\right)$.

Proof Recall the auxiliary Hilbert manifold structure on $\mathcal{V}$ from 5.11 given by the bijection (27). The tangent space at $(a, \gamma) \in A \times H^{s}(\Gamma, M)$ is the set of pairs $(\hat{a}, \hat{\gamma})$ with $\hat{a} \in T_{a} A$ and $\hat{\gamma} \in H^{s}\left(\Gamma, \gamma^{*} T M\right)$. We abuse notation and write

$$
T_{(a, \beta)} \mathcal{V}=T_{a} A \times H^{s}\left(\Gamma, \gamma^{*} T M\right), \quad \gamma:=\beta \circ \iota_{a}
$$

Below we prove the following.

Claim If $\hat{\gamma} \in \Omega^{0}\left(\Gamma, \gamma_{0}^{*} T M\right)$ is a smooth vector field along $\gamma_{0}:=\beta_{0} \circ \iota_{a_{0}}$ then the pair $(0, \hat{\gamma})$ belongs to the sum $T_{\left(a_{0}, \beta_{0}\right)} \mathcal{V}^{\prime}+T_{\left(a_{0}, \beta_{0}\right)} \mathcal{V}^{\prime \prime}$.

We show first that this claim implies (i). By part (ii) of Theorem 5.12 the sum $T_{\beta_{0}} \mathcal{V}_{a_{0}}^{\prime}+$ $T_{\beta_{0}} \mathcal{V}_{a_{0}}^{\prime \prime}$ is a closed subspace of $T_{\beta_{0}} \mathcal{V}_{a_{0}}$ and hence $T_{\left(a_{0}, \beta_{0}\right)} \mathcal{V}^{\prime}+T_{\left(a_{0}, \beta_{0}\right)} \mathcal{V}^{\prime \prime}$ is a closed subspace of $T_{\left(a_{0}, \beta_{0}\right)} \mathcal{V}$. Hence the claim implies that every vertical tangent vector $(0, \hat{\gamma})$ with $\hat{\gamma} \in H^{s}\left(\Gamma, \gamma^{*} T M\right)$ is contained in the sum $T_{\left(a_{0}, \beta_{0}\right)} \mathcal{V}^{\prime}+T_{\left(a_{0}, \beta_{0}\right)} \mathcal{V}^{\prime \prime}$. Since the projection $\mathcal{V}^{\prime} \rightarrow A$ is a submersion by part (iv), this implies

$$
T_{\left(a_{0}, \beta_{0}\right)} \mathcal{V}^{\prime}+T_{\left(a_{0}, \beta_{0}\right)} \mathcal{V}^{\prime \prime}=T_{\left(a_{0}, \beta_{0}\right)} \mathcal{V}
$$

Thus we have proved that (i) follows from the claim.

The desingularization $w_{0}: \Sigma \rightarrow P_{a_{0}}$ induces a decomposition

$$
\Sigma=\Sigma^{\prime} \cup \Sigma^{\prime \prime}, \quad \Sigma^{\prime}:=w_{0}^{-1}\left(P_{a_{0}}^{\prime}\right), \quad \Sigma^{\prime \prime}:=w_{0}^{-1}\left(P_{a_{0}}^{\prime \prime}\right) .
$$

The intersection $\Sigma^{\prime} \cap \Sigma^{\prime \prime}=\partial \Sigma^{\prime}=\partial \Sigma^{\prime \prime}$ is diffeomorphic to the 1-manifold $\Gamma$ in (26). To simplify the notation we assume that

$$
\Gamma=\Sigma^{\prime} \cap \Sigma^{\prime \prime}
$$

The core admits a smooth desingularization

$$
\iota: A_{0} \times \Sigma \rightarrow P_{0}:=\pi_{A}^{-1}\left(A_{0}\right)
$$

that agrees with $w_{0}: \Sigma \rightarrow P_{a_{0}}$ at the base point $a_{0}$ and with the trivialization (26) on $A_{0} \times \Gamma$. Choose $\iota$ so that it maps each component of $A_{0} \times \cup v$ to the corresponding component $C_{\mathrm{i}}$ of the singular set and so that

$$
\iota^{-1}\left(R_{\mathrm{i}}\right)=A_{0} \times\left\{s_{0, \mathrm{i}}\right\}, \quad \mathrm{i}=1, \ldots, \mathrm{n} .
$$

For $a \in A_{0}$ define the desingularization $\iota_{a}: \Sigma \rightarrow P_{a}$ by

$$
\iota_{a}(z):=\iota(a, z)
$$


The trivialization induces a map $j: A_{0} \rightarrow \mathcal{J}(\Sigma)$ determined by the condition that $\iota_{a}$ is holomorphic with respect to $j(a)$ for every $a \in A_{0}$. Since $\left(\pi_{A}, R_{*}, a_{0}\right)$ is a universal unfolding as in [4], the map $j: A \rightarrow \mathcal{J}(\Sigma)$ contains a local slice of the $\operatorname{Diff}(\Sigma)$-action.

We prove the claim. Let $\hat{\gamma} \in \Omega^{0}\left(\Gamma, \gamma_{0}^{*} T M\right)$ be a smooth vector field along $\gamma_{0}$. There exist $\xi^{\prime} \in \Omega^{0}\left(\Sigma^{\prime}, v_{0}^{*} T M\right), \xi^{\prime \prime} \in \Omega^{0}\left(\Sigma^{\prime \prime}, v_{0}^{*} T M\right)$, and $\eta \in \Omega^{0,1}\left(\Sigma, v_{0}^{*} T M\right)$ such that

$$
\hat{\gamma}=\left(\xi^{\prime}-\xi^{\prime \prime}\right)\left|\Gamma, \quad D_{w_{0}} \xi^{\prime}=\eta\right| \Sigma^{\prime}, \quad D_{w_{0}} \xi^{\prime \prime}=\eta \mid \Sigma^{\prime \prime} .
$$

To see this take $\xi^{\prime}=0$ and construct $\xi^{\prime \prime}$ so that $D_{w_{0}} \xi^{\prime \prime}$ vanishes to infinite order along $\Gamma$. (The equation determines the Taylor expansion along $\Gamma$ and then use Emile Borel's extension theorem.) By the hypothesis that the stable map $\left(\Sigma, s_{0, *}, v_{0}, j_{0}, v_{0}\right)$ is regular, there exists $\hat{a} \in T_{a_{0}} A$ and $\hat{v} \in \Omega^{0}\left(\Sigma / \nu_{0}, w_{0}^{*} T M\right)$ such that

$$
\eta=\mathcal{D}_{a_{0}, v_{0}}(\hat{a}, \hat{v}):=D_{v_{0}} \hat{v}+\frac{1}{2} d v_{0} \cdot j_{0} d j(a) \hat{a} .
$$

It follows that the pair $\left(\left(\xi^{\prime}-\hat{v}\right) \mid \Gamma,-\hat{a}\right)$ represents a tangent vector to $\mathcal{V}^{\prime}$ and the pair $\left(\left(\xi^{\prime \prime}-\right.\right.$ $\hat{v}) \mid \Gamma,-\hat{a})$ represents a tangent vector to $\mathcal{V}^{\prime \prime}$. Their difference is equal to $(0, \hat{\gamma})$. This proves the claim and hence part (i) of the theorem.

We prove (ii). By (i) and Theorem 5.12 (ii), the intersection $\mathcal{V}^{\prime} \cap \mathcal{V}^{\prime \prime}$ has complex dimension

$$
\begin{aligned}
\operatorname{dim}_{\mathbb{C}}\left(\mathcal{V}^{\prime} \cap \mathcal{V}^{\prime \prime}\right) & =\operatorname{index}_{\mathbb{C}}\left(D_{v_{0}}\right)+\operatorname{dim}_{\mathbb{C}}(A) \\
& =(\mathrm{m}-3)(1-\mathrm{g})+\left\langle c_{1}, \mathrm{~d}\right\rangle+\mathrm{n}
\end{aligned}
$$

where $\mathrm{d}:=\left[v_{0}\right] \in H_{2}(M ; \mathbb{Z})$ denotes the homology class represented by $v_{0}$. Now abbreviate

$$
\gamma_{0}:=v_{0} \mid \Gamma=\beta_{0} \circ \iota_{a_{0}}: \Gamma \rightarrow M .
$$

Assertion (ii) follows from the fact that the subspace

$$
\mathcal{X}_{0}:=\left\{(\hat{a}, \hat{\gamma}) \in T_{\left(a_{0}, \beta_{0}\right)} \mathcal{V}^{\prime} \cap T_{\left(a_{0}, \beta_{0}\right)} \mathcal{V}^{\prime \prime} \mid \hat{a} \in T_{a_{0}} A_{0}\right\}
$$

has dimension

$$
\operatorname{dim}_{\mathbb{C}} \mathcal{X}_{0}=(\mathrm{m}-3)(1-\mathrm{g})+\left\langle c_{1}, \mathrm{~d}\right\rangle+\mathrm{n}-\mathrm{k} .
$$

To prove this we observe that the pair $(\hat{a}, \hat{\gamma}) \in T_{a_{0}} A \times \Omega^{0}\left(\Gamma, \gamma_{0}^{*} T M\right)$ belongs to the intersection $T_{\left(a_{0}, \beta_{0}\right)} \mathcal{V}^{\prime} \cap T_{\left(a_{0}, \beta_{0}\right)} \mathcal{V}^{\prime \prime}$ if and only if there exists a vector field $\hat{v} \in \Omega^{0}\left(\Sigma / \nu, v_{0}^{*} T M\right)$ satisfying

$$
\mathcal{D}_{a_{0}, v_{0}}(\hat{a}, \hat{v})=D_{v_{0}} \hat{v}+\frac{1}{2} d v_{0} \cdot j_{0} d j(a) \hat{a}=0, \quad \hat{v} \mid \Gamma=\hat{\gamma} .
$$

Since the restriction of the operator

$$
D_{v_{0}}: \Omega^{0}\left(\Sigma / \nu, v_{0}^{*} T M\right) \rightarrow \Omega^{0,1}\left(\Sigma, v_{0}^{*} T M\right)
$$

is Fredholm with index

$$
\operatorname{index}_{\mathbb{C}}\left(D_{v_{0}}\right)=\mathrm{m}(1-\mathrm{g})+\left\langle c_{1}, \mathrm{~d}\right\rangle
$$

and

$$
\operatorname{dim}_{\mathbb{C}} A_{0}=3 \mathrm{~g}-3+\mathrm{n}-\mathrm{k}
$$

and the augmented operator

$$
\mathcal{D}_{a_{0}, v_{0}}: T_{a_{0}} A_{0} \times \Omega^{0}\left(\Sigma / v, v_{0}^{*} T M\right) \rightarrow \Omega^{0,1}\left(\Sigma, v_{0}^{*} T M\right)
$$

is surjective, this implies (32) and hence part (ii) of the theorem. 
5.17 For every $a \in A$ there is a map

$$
\mathcal{U}_{a} \rightarrow \mathcal{V}_{a}:(\alpha, b) \mapsto \beta:=H_{B} \circ \alpha
$$

which sends $\mathcal{U}_{a}^{\prime}$ to $\mathcal{V}_{a}^{\prime}$ and $\mathcal{U}_{a}^{\prime \prime}$ to $\mathcal{V}_{a}^{\prime \prime}$. It follows from our definitions and Theorems 5.9 and 5.12 that the unfolding $\left(\pi_{B}, S_{*}, H_{B}, b\right)$ is infinitesimally universal if and only if the operator

$$
d H_{B}(\alpha): T_{(\alpha, b)} \mathcal{U}_{a} \rightarrow T_{\beta} \mathcal{V}_{a}
$$

induces isomorphisms

$$
\begin{aligned}
& d H_{B}(\alpha): T_{(\alpha, b)} \mathcal{U}_{a}^{\prime} \cap T_{(\alpha, b)} \mathcal{U}_{a}^{\prime \prime} \rightarrow T_{\beta} \mathcal{V}_{a}^{\prime} \cap T_{\beta} \mathcal{V}_{a}^{\prime \prime}, \\
& d H_{B}(\alpha): \frac{T_{(\alpha, b)} \mathcal{U}_{a}}{T_{(\alpha, b)} \mathcal{U}_{a}^{\prime}+T_{(\alpha, b)} \mathcal{U}_{a}^{\prime \prime}} \rightarrow \frac{T_{\beta} \mathcal{V}_{a}}{T_{\beta} \mathcal{V}_{a}^{\prime}+T_{\beta} \mathcal{V}_{a}^{\prime \prime}}
\end{aligned}
$$

for some (and hence every) unfolding $\left(\pi_{A}, R_{*}, H_{A}, a\right)$ and fiber isomorphism $f: P_{a} \rightarrow Q_{b}$. Thus (33) is an exact morphism of Fredholm quadruples as in 6.5 below.

\section{Fredholm intersection theory}

6.1 Let $E$ be a Hilbert space and $E^{\prime}, E^{\prime \prime} \subset E$ be closed subspaces. We call $\left(E, E^{\prime}, E^{\prime \prime}\right)$ a Fredholm triple (of subspaces) if the intersection $E^{\prime} \cap E^{\prime \prime}$ is finite dimensional, the sum $E^{\prime}+E^{\prime \prime}$ is a closed subspace of $E$, and the quotient $E /\left(E^{\prime}+E^{\prime \prime}\right)$ is finite dimensional. The triple $\left(E, E^{\prime}, E^{\prime \prime}\right)$ is Fredholm if and only if the operator

$$
E^{\prime} \times E^{\prime \prime} \rightarrow E:\left(x^{\prime}, x^{\prime \prime}\right) \mapsto x^{\prime}+x^{\prime \prime}
$$

is Fredholm. The Fredholm index of the triple is defined as the Fredholm index of the operator (34). The image of (34) is the sum $E^{\prime}+E^{\prime \prime}$ and its kernel is isomorphic to $E^{\prime} \cap E^{\prime \prime}$ via the inclusion

$$
E^{\prime} \cap E^{\prime \prime} \rightarrow E^{\prime} \times E^{\prime \prime}: x \mapsto(x,-x) .
$$

Hence the index of the triple $\left(E, E^{\prime}, E^{\prime \prime}\right)$ is

$$
\operatorname{index}\left(E, E^{\prime}, E^{\prime \prime}\right):=\operatorname{dim}\left(E^{\prime} \cap E^{\prime \prime}\right)-\operatorname{dim}\left(E /\left(E^{\prime}+E^{\prime \prime}\right)\right) .
$$

Standard Fredholm theory implies that the Fredholm property and the index are stable under small deformations of the subspaces $E^{\prime}$ and $E^{\prime \prime}$.

6.2 Let $X$ be a Hilbert manifold, $X^{\prime}, X^{\prime \prime} \subset X$ be smooth submanifolds, and $x_{0} \in X^{\prime} \cap X^{\prime \prime}$. We call the quadruple $\left(X, X^{\prime}, X^{\prime \prime}, x_{0}\right)$ Fredholm if the triple $\left(T_{x_{0}} X, T_{x_{0}} X^{\prime}, T_{x_{0}} X^{\prime \prime}\right)$ is Fredholm. Define its Fredholm index to be the index of the triple. If $\left(X, X^{\prime}, X^{\prime \prime}, x_{0}\right)$ is Fredholm then so is $\left(X, X^{\prime}, X^{\prime \prime}, x\right)$ for $x \in X^{\prime} \cap X^{\prime \prime}$ sufficiently close to $x_{0}$ and both quadruples have the same Fredholm index.

Lemma 6.3 (Normal coordinates) Let $\left(X, X^{\prime}, X^{\prime \prime}, x_{0}\right)$ be a Fredholm quadruple as in 6.2 and abbreviate

$$
E:=T_{x_{0}} X, \quad E^{\prime}:=T_{x_{0}} X^{\prime}, \quad E^{\prime \prime}:=T_{x_{0}} X^{\prime \prime} .
$$

Then there are coordinates $u, x^{\prime}, x^{\prime \prime}, \xi$ defined in a neighborhood of $x_{0}$ in $X$ satisfying the following conditions.

(i) $u$ takes values in $E^{\prime} \cap E^{\prime \prime}$ and $u\left(x_{0}\right)=0$. 
(ii) $x^{\prime}$ takes values in a complement to $E^{\prime} \cap E^{\prime \prime}$ in $E^{\prime}$ and $x^{\prime}\left(x_{0}\right)=0$.

(iii) $x^{\prime \prime}$ takes values in a complement to $E^{\prime} \cap E^{\prime \prime}$ in $E^{\prime \prime}$ and $x^{\prime \prime}\left(x_{0}\right)=0$.

(iv) $\xi$ takes values in a complement to $E^{\prime}+E^{\prime \prime}$ in $E$ and $\xi\left(x_{0}\right)=0$.

(v) Near $x_{0}$ the submanifolds $X^{\prime}, X^{\prime \prime}$ and the subset $X^{\prime} \cap X^{\prime \prime}$ are given by

$$
\begin{gathered}
X^{\prime \prime}=\left\{x^{\prime}=0, \xi=0\right\}, \quad X^{\prime}=\left\{x^{\prime \prime}=0, \xi=f\left(u, x^{\prime}\right)\right\}, \\
X^{\prime} \cap X^{\prime \prime}=\left\{x^{\prime}=0, x^{\prime \prime}=0, \xi=0, f(u, 0)=0\right\}
\end{gathered}
$$

for a smooth function $f$ with $f(0,0)=0$ and $d f(0,0)=0$.

Proof Choose any coordinate chart $\left(X^{\prime \prime}, x_{0}\right) \rightarrow\left(E^{\prime \prime}, 0\right)$ whose differential at $x_{0}$ is the identity. This coordinate chart can be written as $\left(u, x^{\prime \prime}\right)$ where $u$ takes values in $E^{\prime} \cap E^{\prime \prime}$ and $x^{\prime \prime}$ takes values in a complement of $E^{\prime} \cap E^{\prime \prime}$ in $E^{\prime \prime}$. Extend $\left(u, x^{\prime \prime}\right)$ to a coordinate chart $\left(X, x_{0}\right) \rightarrow(E, 0)$. This extended coordinate chart can be written as $\left(u, x^{\prime}, x^{\prime \prime}, \xi\right)$ where $x^{\prime}$ takes values in a complement of $E^{\prime} \cap E^{\prime \prime}$ in $E^{\prime}$ and $\xi$ takes values in a complement of $E^{\prime}+E^{\prime \prime}$ in $E$. In these coordinates we have

$$
X^{\prime \prime}=\left\{x^{\prime}=0, \xi=0\right\}, \quad X^{\prime}=\left\{x^{\prime \prime}=\phi\left(u, x^{\prime}\right), \xi=f\left(u, x^{\prime}\right)\right\} .
$$

where $\phi(0,0)=0, d \phi(0,0)=0$ and $f(0,0)=0, d f(0,0)=0$. Now replace $x^{\prime \prime}$ by $x^{\prime \prime}-\phi\left(u, x^{\prime}\right)$ to obtain the required coordinate system.

Corollary 6.4 Let $\left(X, X^{\prime}, X^{\prime \prime}, x_{0}\right)$ be as in Lemma 6.3. Then there exists a neighborhood $X_{0}$ of $x_{0}$ in $X$ and finite dimensional submanifolds $U, U^{\prime}, U^{\prime \prime}$ of $X, X^{\prime}, X^{\prime \prime}$, respectively, passing through $x_{0}$ such that

$$
U^{\prime}=U \cap X^{\prime}, \quad U^{\prime \prime}=U \cap X^{\prime \prime}, \quad U^{\prime} \cap U^{\prime \prime}=X_{0} \cap X^{\prime} \cap X^{\prime \prime}
$$

and, for $x \in U^{\prime} \cap U^{\prime \prime}$, we have

$$
T_{x} U^{\prime} \cap T_{x} U^{\prime \prime}=T_{x} X^{\prime} \cap T_{x} X^{\prime \prime}, \quad \frac{T_{x} U}{T_{x} U^{\prime}+T_{x} U^{\prime \prime}} \cong \frac{T_{x} X}{T_{x} X^{\prime}+T_{x} X^{\prime \prime}} .
$$

We call $\left(U, U^{\prime}, U^{\prime \prime}, x_{0}\right)$ a finite dimensional reduction.

Proof Let $X_{0}$ be the domain of the normal form coordinates $u, x^{\prime}, x^{\prime \prime}, \xi$ introduced in Lemma 6.3. Then

$$
\begin{gathered}
X_{0} \cap X^{\prime} \cap X^{\prime \prime}=\{(u, 0,0,0) \mid f(u, 0)=0\}, \\
T_{x} X^{\prime} \cap T_{x} X^{\prime \prime}=\{(\hat{u}, 0,0,0) \mid d f(u, 0)(\hat{u}, 0)=0\}, \\
T_{x} X^{\prime}+T_{x} X^{\prime \prime}=\left\{\left(\hat{u}, \hat{x}^{\prime}, \hat{x}^{\prime \prime}, \hat{\xi}\right) \mid \hat{\xi}-\frac{\partial f}{\partial x^{\prime}} \hat{x}^{\prime} \in \operatorname{im} \frac{\partial f}{\partial u}\right\}
\end{gathered}
$$

for $x=(u, 0,0,0) \in X_{0} \cap X^{\prime} \cap X^{\prime \prime}$. Hence the submanifolds

$$
U:=\{(u, 0,0, \xi)\}, \quad U^{\prime}:=\{(u, 0,0, f(u, 0))\}, \quad U^{\prime \prime}:=\{(u, 0,0,0)\}
$$

satisfy the requirements of the corollary. 
6.5 A morphism from $\left(X, X^{\prime}, X^{\prime \prime}, x_{0}\right)$ to $\left(Y, Y^{\prime}, Y^{\prime \prime}, y_{0}\right)$ is a smooth map $h: X \rightarrow Y$ such that

$$
h\left(X^{\prime}\right) \subset Y^{\prime}, \quad h\left(X^{\prime \prime}\right) \subset Y^{\prime \prime}, \quad h\left(x_{0}\right)=y_{0} .
$$

The morphism $h$ is called exact (at $x_{0}$ ) if the differential $d h\left(x_{0}\right): T_{x_{0}} X \rightarrow T_{y_{0}} Y$ induces isomorphisms

$$
d h\left(x_{0}\right): T_{x_{0}} X^{\prime} \cap T_{x_{0}} X^{\prime \prime} \rightarrow T_{y_{0}} Y^{\prime} \cap T_{y_{0}} Y^{\prime \prime}
$$

and

$$
d h\left(x_{0}\right): \frac{T_{x_{0}} X}{T_{x_{0}} X^{\prime}+T_{x_{0}} X^{\prime \prime}} \rightarrow \frac{T_{y_{0}} Y}{T_{y_{0}} Y^{\prime}+T_{y_{0}} Y^{\prime \prime}} .
$$

The inclusion of a finite dimensional reduction is an example of an exact morphism.

Theorem 6.6 Let $h:\left(X, X^{\prime}, X^{\prime \prime}, x_{0}\right) \rightarrow\left(Y, Y^{\prime}, Y^{\prime \prime}, y_{0}\right)$ be a morphism of Fredholm quadruples. Then the following are equivalent.

(i) $h$ is exact at $x_{0}$.

(ii) There exist finite dimensional reductions $\left(U, U^{\prime}, U^{\prime \prime}, x_{0}\right)$ of $\left(X, X^{\prime}, X^{\prime \prime}, x_{0}\right)$ and $\left(V, V^{\prime}, V^{\prime \prime}, y_{0}\right)$ of $\left(Y, Y^{\prime}, Y^{\prime \prime}, y_{0}\right)$ such that $h$ maps $U, U^{\prime}, U^{\prime \prime}$ diffeomorphically onto $V, V^{\prime}, V^{\prime \prime}$, respectively.

Proof We prove that (ii) implies (i). By (ii), the homomorphism $d h\left(x_{0}\right)$ from $T_{x_{0}} X^{\prime} \cap T_{x_{0}} X^{\prime \prime}$ to $T_{y_{0}} Y^{\prime} \cap T_{y_{0}} Y^{\prime \prime}$ can be written as the composition

$$
T_{x_{0}} X^{\prime} \cap T_{x_{0}} X^{\prime \prime}=T_{x_{0}} U^{\prime} \cap T_{x_{0}} U^{\prime \prime} \stackrel{d h\left(x_{0}\right)}{\longrightarrow} T_{y_{0}} V^{\prime} \cap T_{y_{0}} V^{\prime \prime}=T_{y_{0}} Y^{\prime} \cap T_{y_{0}} Y^{\prime \prime}
$$

and hence is an isomorphism. Similarly for the map from $T_{x_{0}} X /\left(T_{x_{0}} X^{\prime}+T_{x_{0}} X^{\prime \prime}\right)$ to $T_{y_{0}} Y /$ $\left(T_{y_{0}} Y^{\prime}+T_{y_{0}} Y^{\prime \prime}\right)$.

We prove that (i) implies (ii). Let $u, x^{\prime}, x^{\prime \prime}, \xi$ be the normal coordinates on $X$ introduced in Lemma 6.3 and choose similar normal coordinates $v, y^{\prime}, y^{\prime \prime}, \eta$ on $Y$ at $y_{0}$. Thus

$$
\begin{gathered}
Y^{\prime \prime}=\left\{y^{\prime}=0, \eta=0\right\}, \quad Y^{\prime}=\left\{y^{\prime \prime}=0, \eta=g\left(v, y^{\prime}\right)\right\}, \\
Y^{\prime} \cap Y^{\prime \prime}=\left\{y^{\prime}=0, y^{\prime \prime}=0, \eta=0, g(v, 0)=0\right\}
\end{gathered}
$$

for a smooth function $g$ with $g(0,0)=0$ and $d g(0,0)=0$. In these coordinates the morphism $h=\left(h_{1}, h_{2}, h_{3}, h_{4}\right)$ satsfies

$$
h_{2}\left(u, 0, x^{\prime \prime}, 0\right)=0, \quad h_{4}\left(u, 0, x^{\prime \prime}, 0\right)=0
$$

(because $\left.h\left(X^{\prime \prime}\right) \subset Y^{\prime \prime}\right)$,

$$
\begin{gathered}
h_{3}\left(u, x^{\prime}, 0, f\left(u, x^{\prime}\right)\right)=0, \\
h_{4}\left(u, x^{\prime}, 0, f\left(u, x^{\prime}\right)\right)=g\left(h_{1}\left(u, x^{\prime}, 0, f\left(u, x^{\prime}\right)\right), h_{2}\left(u, x^{\prime}, 0, f\left(u, x^{\prime}\right)\right)\right)
\end{gathered}
$$

(because $\left.h\left(X^{\prime}\right) \subset Y^{\prime}\right)$, and

$$
\operatorname{det}\left(\partial h_{1} / \partial u\right)(0,0,0,0) \neq 0, \quad \operatorname{det}\left(\partial h_{4} / \partial \xi\right)(0,0,0,0) \neq 0
$$

(because $h$ is exact). By (38) and (41), the restriction of $h$ to a neighborhood of $x_{0}$ in $U$ is an embedding. Shrinking the domain $X_{0} \subset X$ of the normal coordinates, if necessary, we may assume that $h \mid U: U \rightarrow Y$ is an embedding. Denote

$$
V:=h(U), \quad V^{\prime}:=h\left(U^{\prime}\right), \quad V^{\prime \prime}:=h\left(U^{\prime \prime}\right) .
$$

We must prove that $\left(V, V^{\prime}, V^{\prime \prime}, y_{0}\right)$ is a finite dimensional reduction. 
(a) The set $V$ consists of all quadruples of the form $\left(v, y^{\prime}, y^{\prime \prime}, \eta\right)$ where

$$
y^{\prime}:=h_{2}(u, 0,0, \xi), \quad y^{\prime \prime}:=h_{3}(u, 0,0, \xi)
$$

and $u, \xi$ are defined by $h_{1}(u, 0,0, \xi)=v, h_{4}(u, 0,0, \xi)=\eta$.

(b) The set $V^{\prime}$ consists of all quadruples of the form $\left(v, y^{\prime}, 0, g\left(v, y^{\prime}\right)\right)$ where

$$
y^{\prime}:=h_{2}(u, 0,0, f(u, 0)), \quad h_{1}(u, 0,0, f(u, 0)):=v .
$$

(c) The set $V^{\prime \prime}$ consists of all quadruples of the form $\left(v, 0, y^{\prime \prime}, 0\right)$ where

$$
y^{\prime \prime}:=h_{3}(u, 0,0,0), \quad h_{1}(u, 0,0,0):=v .
$$

Thus a point in the intersection $V^{\prime} \cap V^{\prime \prime}$ has the form $(v, 0,0,0)$ where $v$ satisfies the conditions

(i) $g(v, 0)=0$

(ii) If $u$ is defined by $h_{1}(u, 0,0, f(u, 0)):=v$ then $h_{2}(u, 0,0, f(u, 0))=0$.

(iii) If $u$ is defined by $h_{1}(u, 0,0,0):=v$ then $h_{3}(u, 0,0,0)=0$.

We show that (i) implies (ii) and (iii) whenever $v$ is sufficiently small. For (ii) we define $u$ as the unique solution of $h_{1}(u, 0,0, f(u, 0))=v$ so that

$$
g(v, 0)=0, \quad g\left(v, h_{2}(u, 0,0, f(u, 0))\right)=h_{4}(u, 0,0, f(u, 0)) .
$$

We claim that for $v$ sufficiently small this implies $f(u, 0)=0$. To see this we use first that the solution $u$ of the equation $h_{1}(u, 0,0, f(u, 0))=v$ satisfies an inequality

$$
\|u\|+\|f(u, 0)\| \leq c\|v\|
$$

for $v$ sufficiently small. Next we use the fact that $h_{2}(u, 0,0,0)=0$ and hence

$$
\left\|h_{2}(u, 0,0, \xi)\right\| \leq c\|\xi\| .
$$

Third, we have that $h_{4}(u, 0,0,0)=0$ and $\partial h_{4} / \partial \xi$ is invertible at the point $(0,0,0,0)$, hence also at the point $(u, 0,0,0)$ for $u$ sufficiently small. Hence we have an inequality

$$
\left\|h_{4}(u, 0,0, \xi)\right\| \geq c^{-1}\|\xi\|
$$

for a suitable constant $c>0$ and $u$ and $\xi$ sufficiently small. Fourth, since $g(0,0)=0$ and $d g(0,0)=0$, there is an inequality

$$
\left\|g\left(v, y^{\prime}\right)-g(v, 0)\right\| \leq c\left(\|v\|+\left\|y^{\prime}\right\|\right)\left\|y^{\prime}\right\|
$$

for a suitable constant $c$. Putting these four inequalities together and inserting $\xi=f(u, 0)$ and $y^{\prime}=h_{2}(u, 0,0, f(u, 0))$ we deduce

$$
\begin{array}{rlr}
\|f(u, 0)\| & \leq c\left\|h_{4}(u, 0,0, f(u, 0))\right\| & \text { by (45) } \\
& =c\left\|g\left(v, h_{2}(u, 0,0, f(u, 0))\right)-g(v, 0)\right\| & \text { by (42) } \\
& \leq c^{2}\left(\|v\|+\left\|h_{2}(u, 0,0, f(u, 0))\right\|\right) \| h_{2}(u, 0,0, f(u, 0)) & \text { by (46) } \\
& \leq c^{3}(\|v\|+c\|f(u, 0)\|)\|f(u, 0)\| & \text { by (44) } \\
& \leq\left(c^{3}+c^{5}\right)\|v\|\|f(u, 0)\| & \text { by (43) }
\end{array}
$$

for $v$ sufficiently small. With $\left(c^{3}+c^{5}\right)\|v\|<1$ this implies

$$
f(u, 0)=0
$$

as claimed and hence $h_{2}(u, 0,0, f(u, 0))=0$, by (38). Thus we have proved that (i) implies (ii). Since $f(u, 0)=0$ we also deduce that our $u$ is the unique solution of 
$h_{1}(u, 0,0,0)=v$ needed in (iii). Using $f(u, 0)=0$ again we obtain $h_{3}(u, 0,0,0)=0$, by (39). Thus we have proved that (i) implies (ii) and (iii) and hence

$$
V^{\prime} \cap V^{\prime \prime}=\{(v, 0,0,0) \mid g(v, 0)=0\}=Y_{0} \cap Y^{\prime} \cap Y^{\prime \prime}
$$

for a suitable open neighborhood $Y_{0}$ of $y_{0}$ in $Y$.

Next we examine the tangent spaces of $V, V^{\prime}$, and $V^{\prime \prime}$ at a point

$$
y:=(v, 0,0,0) \in V^{\prime} \cap V^{\prime \prime}, \quad g(v, 0)=0 .
$$

Let $x=(u, 0,0,0) \in U^{\prime} \cap U^{\prime \prime}$ with $f(u, 0)=0$ be the element with $h(x)=y$.

(A) The tangent space $T_{y} V$ consists of all vectors $\hat{y}=\left(\hat{v}, \hat{y}^{\prime}, \hat{y}^{\prime \prime}, \hat{\eta}\right)$ where

$$
\hat{y}^{\prime}:=\frac{\partial h_{2}}{\partial \xi} \hat{\xi}, \quad \hat{y}^{\prime \prime}:=\frac{\partial h_{3}}{\partial u} \hat{u}+\frac{\partial h_{3}}{\partial \xi} \hat{\xi}
$$

and $\hat{u}, \hat{\xi}$ are defined by

$$
\begin{gathered}
\hat{u}:=\left(\frac{\partial h_{1}}{\partial u}\right)^{-1}\left(\hat{v}-\frac{\partial h_{1}}{\partial \xi} \hat{\xi}\right) \\
\hat{\xi}:=\left(\frac{\partial h_{4}}{\partial \xi}\right)^{-1} \hat{\eta} .
\end{gathered}
$$

Here and below all partial derivatives of $h$ are evaluated at $x=(u, 0,0,0)$ and we have used the fact that $\partial h_{2} / \partial u$ and $\partial h_{4} / \partial u$ vanish at $x$, by (38).

(B) The tangent space $T_{y} V^{\prime}$ consists of all vectors $\hat{y}=\left(\hat{v}, \hat{y}^{\prime}, 0, \hat{\eta}\right)$ where

$$
\hat{y}^{\prime}:=\frac{\partial h_{2}}{\partial \xi} \frac{\partial f}{\partial u} \hat{u}, \quad \hat{\eta}:=\frac{\partial g}{\partial v} \hat{v}+\frac{\partial g}{\partial y^{\prime}} \hat{y}^{\prime}
$$

and $\hat{u}$ is defined

$$
\hat{u}:=\left(\frac{\partial h_{1}}{\partial u}+\frac{\partial h_{1}}{\partial \xi} \frac{\partial f}{\partial u}\right)^{-1} \hat{v} .
$$

Here and below all partial derivatives of $f$ are evaluated at $(u, 0)$ and all partial derivatives of $g$ at $(v, 0)$.

(C) The tangent space $T_{y} V^{\prime \prime}$ consists of all vectors $\hat{y}=\left(\hat{v}, 0, \hat{y}^{\prime \prime}, 0\right)$ where

$$
\hat{y}^{\prime \prime}:=-\frac{\partial h_{3}}{\partial \xi} \frac{\partial f}{\partial u} \hat{u}, \quad \hat{u}:=\left(\frac{\partial h_{1}}{\partial u}\right)^{-1} \hat{v} .
$$

Note that $-\left(\partial h_{3} / \partial \xi\right)(\partial f / \partial u)=\partial h_{3} / \partial u$, by (39).

We prove that the intersection $T_{y} V^{\prime} \cap T_{y} V^{\prime \prime}$ consists of all vectors $\hat{y}=(\hat{v}, 0,0,0)$ where $\hat{v}$ satisfies the conditions

$$
\begin{aligned}
& \frac{\partial g}{\partial v} \hat{v}=0, \\
& \frac{\partial f}{\partial u} \hat{u}=0
\end{aligned}
$$

where $\hat{u}$ is given by (50). First assume $\hat{v}$ satisfies (52) and (53). We show that $\hat{y}:=$ $(\hat{v}, 0,0,0) \in T_{y} V^{\prime} \cap T_{y} V^{\prime \prime}$. By (53), we have $\hat{y}^{\prime}=0$ in (49) and hence, by (52), $\hat{\eta}=$ 
$(\partial g / \partial v) \hat{v}=0$ in (49). Thus $\hat{y} \in T_{y} V^{\prime}$. Moreover the vector $\hat{u}$ in (50) satisfies $\left(\partial h_{1} / \partial u\right) \hat{u}=\hat{v}$ by (53) and, also by (53), we have $\hat{y}^{\prime \prime}=0$ in (51). Thus $\hat{y} \in T_{y} V^{\prime \prime}$.

Conversely assume $\hat{y} \in T_{y} V^{\prime} \cap T_{y} V^{\prime \prime}$. We show that $\hat{y}=(\hat{v}, 0,0,0)$ where $\hat{v}$ satisfies (52) and (53). That $\hat{y}$ has the form $(\hat{v}, 0,0,0)$ follows immediately from (B) and (C). Equation (52) follows immediately from (B) and the fact that $\hat{y}^{\prime}=0$. To prove that $\hat{v}$ satisfies (53) we differentiate equation (40) at the point $x=(u, 0,0,0)$ with respect to $u$ to obtain

$$
\frac{\partial h_{4}}{\partial \xi} \frac{\partial f}{\partial u}=\frac{\partial g}{\partial v}\left(\frac{\partial h_{1}}{\partial u}+\frac{\partial h_{1}}{\partial \xi} \frac{\partial f}{\partial u}\right)+\frac{\partial g}{\partial y^{\prime}} \frac{\partial h_{2}}{\partial \xi} \frac{\partial f}{\partial u} .
$$

Here we have used the fact that $\partial h_{2} / \partial u$ and $\partial h_{4} / \partial u$ vanish at $x$, by (38). Evaluating (54) in the direction of the vector $\hat{u}$ in (53) gives

$$
\frac{\partial h_{4}}{\partial \xi} \frac{\partial f}{\partial u} \hat{u}=\frac{\partial g}{\partial v} \hat{v}+\frac{\partial g}{\partial y^{\prime}} \hat{y}^{\prime}=0 .
$$

Since $\partial h_{4} / \partial \xi$ is invertible this proves (53).

We prove that

$$
T_{y} V^{\prime} \cap T_{y} V^{\prime \prime}=\left\{(\hat{v}, 0,0,0) \mid \frac{\partial g}{\partial v} \hat{v}=0\right\},
$$

i.e. that (52) implies (53). Let $\hat{u}$ be given by (50) and abbreviate

$$
\hat{\xi}:=\frac{\partial f}{\partial u} \hat{u}
$$

Evaluating (54) again in the direction of the vector $\hat{u}$ in (53) and using (52) we obtain

$$
\frac{\partial h_{4}}{\partial \xi} \hat{\xi}=\frac{\partial g}{\partial y^{\prime}} \frac{\partial h_{2}}{\partial \xi} \hat{\xi}
$$

Since $\partial g / \partial y^{\prime}$ vanishes at the origin it is small when $v$ is small and hence, in this case, $\hat{\xi}=0$ as claimed. This proves (55). By (36), the right hand side of (55) is $T_{y} Y^{\prime} \cap T_{y} Y^{\prime \prime}$. This proves that

$$
T_{y} V^{\prime} \cap T_{y} V^{\prime \prime}=T_{y} Y^{\prime} \cap T_{y} Y^{\prime \prime} .
$$

It remains to prove that

$$
\frac{T_{y} V}{T_{y} V^{\prime}+T_{y} V^{\prime \prime}} \cong \frac{T_{y} Y}{T_{y} Y^{\prime}+T_{y} Y^{\prime \prime}} .
$$

Since $T_{y} V^{\prime} \cap T_{y} V^{\prime \prime}=T_{y} Y^{\prime} \cap T_{y} Y^{\prime \prime}$ and the Fredholm quadruples $\left(V, V^{\prime}, V^{\prime \prime}, y\right)$ and $\left(Y, Y^{\prime}, Y^{\prime \prime}, y\right)$ have the same Fredholm index for $y \in V^{\prime} \cap V^{\prime \prime}$ sufficiently small, both quotient spaces have the same dimension. Hence condition (56) is equivalent to

$$
T_{y} V \cap\left(T_{y} Y^{\prime}+T_{y} Y^{\prime \prime}\right) \subset T_{y} V^{\prime}+T_{y} V^{\prime \prime} .
$$

The sum $T_{y} Y^{\prime}+T_{y} Y^{\prime \prime}$ is the set of all vectors $\hat{y}=\left(\hat{v}, \hat{y}^{\prime}, \hat{y}^{\prime \prime}, \hat{\eta}\right)$ that satisfy

$$
\hat{\eta}-\frac{\partial g}{\partial y^{\prime}} \hat{y}^{\prime} \in \operatorname{im}\left(\frac{\partial g}{\partial v}\right) \text {. }
$$

To prove (57) fix a vector $\hat{y}=\left(\hat{v}, \hat{y}^{\prime}, \hat{y}^{\prime \prime}, \hat{\eta}\right) \in T_{y} V \cap\left(T_{y} Y^{\prime}+T_{y} Y^{\prime \prime}\right)$. By (58) there is a vector $\hat{v}^{\prime}$ such that

$$
\hat{\eta}-\frac{\partial g}{\partial y^{\prime}} \hat{y}^{\prime}=\frac{\partial g}{\partial v} \hat{v}^{\prime}
$$


We prove that

$$
\left(\hat{v}^{\prime}, \hat{y}^{\prime}, 0, \hat{\eta}\right) \in T_{y} V^{\prime}, \quad\left(\hat{v}^{\prime \prime}, 0, y^{\prime \prime}, 0\right) \in T_{y} V^{\prime \prime}, \quad \hat{v}^{\prime \prime}:=\hat{v}-\hat{v}^{\prime} .
$$

To see this define the vectors $\hat{u}$ and $\hat{\xi}$ by

$$
\frac{\partial h_{1}}{\partial u} \hat{u}+\frac{\partial h_{1}}{\partial \xi} \hat{\xi}=\hat{v}, \quad \frac{\partial h_{4}}{\partial \xi} \hat{\xi}=\hat{\eta}
$$

as in (A) so that

$$
\hat{y}^{\prime}=\frac{\partial h_{2}}{\partial \xi} \hat{\xi}, \quad \hat{y}^{\prime \prime}=\frac{\partial h_{3}}{\partial u} \hat{u}+\frac{\partial h_{3}}{\partial \xi} \hat{\xi}
$$

Next define $\hat{u}^{\prime}$ and $\hat{u}^{\prime \prime}$ by

$$
\frac{\partial h_{1}}{\partial u} \hat{u}^{\prime}+\frac{\partial h_{1}}{\partial \xi} \frac{\partial f}{\partial u} \hat{u}^{\prime}:=\hat{v}^{\prime}, \quad \frac{\partial h_{1}}{\partial u} \hat{u}^{\prime \prime}:=\hat{v}^{\prime \prime} .
$$

Then, by (54), (59), and (61-63), we have

$$
\begin{aligned}
\frac{\partial h_{4}}{\partial \xi}\left(\frac{\partial f}{\partial u} \hat{u}^{\prime}-\hat{\xi}\right) & =\frac{\partial g}{\partial v}\left(\frac{\partial h_{1}}{\partial u}+\frac{\partial h_{1}}{\partial \xi} \frac{\partial f}{\partial u}\right) \hat{u}^{\prime}+\frac{\partial g}{\partial y^{\prime}} \frac{\partial h_{2}}{\partial \xi} \frac{\partial f}{\partial u} \hat{u}^{\prime}-\hat{\eta} \\
& =\frac{\partial g}{\partial v} \hat{v}^{\prime}+\frac{\partial g}{\partial y^{\prime}} \hat{y}^{\prime}-\hat{\eta}+\frac{\partial g}{\partial y^{\prime}} \frac{\partial h_{2}}{\partial \xi}\left(\frac{\partial f}{\partial u} \hat{u}^{\prime}-\hat{\xi}\right) \\
& =\frac{\partial g}{\partial y^{\prime}} \frac{\partial h_{2}}{\partial \xi}\left(\frac{\partial f}{\partial u} \hat{u}^{\prime}-\hat{\xi}\right) .
\end{aligned}
$$

Since $\partial g / \partial y^{\prime}$ is small when $v$ is small this implies

$$
\hat{\xi}=\frac{\partial f}{\partial u} \hat{u}^{\prime}, \quad \hat{u}^{\prime}+\hat{u}^{\prime \prime}=\hat{u} .
$$

Here the last equation follows from the first and (61) and (63). Now it follows from (62) that

$$
\hat{y}^{\prime \prime}=\frac{\partial h_{3}}{\partial u} \hat{u}+\frac{\partial h_{3}}{\partial \xi} \hat{\xi}=\left(\frac{\partial h_{3}}{\partial u}+\frac{\partial h_{3}}{\partial \xi} \frac{\partial f}{\partial u}\right) \hat{u}^{\prime}+\frac{\partial h_{3}}{\partial u} \hat{u}^{\prime \prime}=\frac{\partial h_{3}}{\partial u} \hat{u}^{\prime \prime} .
$$

Combining this with (C) and (63) we find that $\left(\hat{v}^{\prime \prime}, 0, \hat{y}^{\prime \prime}, 0\right) \in T_{y} V^{\prime \prime}$. Likewise it follows from (B) and (59), (62) and (63) that $\left(\hat{v}^{\prime}, \hat{y}^{\prime}, 0, \eta^{\prime}\right) \in T_{y} V^{\prime}$. Thus we have proved (60). This completes the proof of (57) and the theorem.

Let $A \subset X$ and $B \subset Y$ be arbitrary subsets. Recall that $\phi: A \rightarrow B$ is by definition a diffeomorpism if it is bijective and $\phi$ and $\phi^{-1}$ are smooth, i.e. for every point $x \in A$ there is a smooth extension of $\phi$ from a neighbourhood of $x$ in $X$ to $Y$, and for every point $y \in B$ there is a smooth extension of $\phi^{-1}$ from a neighbourhood of $y$ in $Y$ to $X$ (see [3]).

Corollary 6.7 Let $h:\left(X, X^{\prime}, X^{\prime \prime}, x_{0}\right) \rightarrow\left(Y, Y^{\prime}, Y^{\prime \prime}, y_{0}\right)$ be an exact morphism of Fredholm quadruples. Then the following holds.

(I) $h$ maps a neighborhood of $x_{0}$ in $X^{\prime} \cap X^{\prime \prime}$ diffeomorphically onto a neighborhood of $y_{0}$ in $Y^{\prime} \cap Y^{\prime \prime}$.

(II) $h$ is exact at every point $x \in X^{\prime} \cap X^{\prime \prime}$ sufficiently close to $x_{0}$.

Proof Of course $X^{\prime} \cap X^{\prime \prime}$ need not be a manifold. Let $\left(U, U^{\prime}, U^{\prime \prime}\right)$ and $\left(V, V^{\prime}, V^{\prime \prime}\right)$ be the finite dimensional reductions of Theorem 6.6. Then assertion (I) follows from the fact that $h^{-1}: V \rightarrow U$ extends to a smooth map from a neighborhood of $V$ to $X$. Assertion (II) follows 
from the equivalence of (i) and (ii) in Theorem 6.6; namely, if (ii) holds for $x_{0}$ then it also holds for every point $x \in X^{\prime} \cap X^{\prime \prime}$ sufficiently close to $x_{0}$ (with the same finite dimensional reductions). This proves the corollary.

Theorem 6.8 Let $h_{\lambda}:\left(X, X_{\lambda}^{\prime}, X_{\lambda}^{\prime \prime}\right) \rightarrow\left(Y, Y_{\lambda}^{\prime}, Y_{\lambda}^{\prime \prime}\right)$ be a smooth family of morphisms of Fredholm triples parametrized by $\lambda \in \Lambda$, where $\Lambda$ is a finite dimensional manifold, i.e. the map

$$
h: \Lambda \times X \rightarrow \Lambda \times Y, \quad h(\lambda, x):=\left(\lambda, h_{\lambda}(x)\right),
$$

is smooth, the sets

$$
X^{\prime}:=\bigsqcup_{\lambda} X_{\lambda}^{\prime}, \quad X^{\prime \prime}:=\bigsqcup_{\lambda} X_{\lambda}^{\prime \prime}
$$

are smooth submanifolds of $\Lambda \times X$, the sets

$$
Y^{\prime}:=\bigsqcup_{\lambda} Y_{\lambda}^{\prime}, \quad Y^{\prime \prime}:=\bigsqcup_{\lambda} Y_{\lambda}^{\prime \prime}
$$

are smooth submanifolds of $\Lambda \times Y$, and the projections from $X^{\prime}, X^{\prime \prime}, Y^{\prime}, Y^{\prime \prime}$ to $\Lambda$ are submersions. Let $\lambda_{0} \in \Lambda, x_{0} \in X_{\lambda_{0}}^{\prime} \cap X_{\lambda_{0}}^{\prime \prime}$, and $y_{0}:=h_{\lambda_{0}}\left(x_{0}\right)$. Then the following holds.

(i) The Fredholm indices are related by

$$
\begin{aligned}
\operatorname{index}\left(\Lambda \times X, X^{\prime}, X^{\prime \prime},\left(\lambda_{0}, x_{0}\right)\right) & =\operatorname{index}\left(X_{\lambda_{0}}, X_{\lambda_{0}}^{\prime}, X_{\lambda_{0}}^{\prime \prime}, x_{0}\right)+\operatorname{dim} \Lambda, \\
\operatorname{index}\left(\Lambda \times Y, Y^{\prime}, Y^{\prime \prime},\left(\lambda_{0}, y_{0}\right)\right) & =\operatorname{index}\left(Y_{\lambda_{0}}, Y_{\lambda_{0}}^{\prime}, Y_{\lambda_{0}}^{\prime \prime}, y_{0}\right)+\operatorname{dim} \Lambda .
\end{aligned}
$$

(ii) $h_{\lambda_{0}}$ is exact at $x_{0}$ if and only if $h$ is exact at $\left(\lambda_{0}, x_{0}\right)$.

Proof There is a commutative diagram

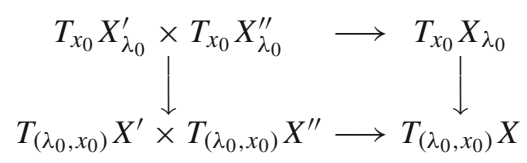

of Fredholm operators where the horizontal arrows are as in 6.1 and the vertical arrows are inclusions. The Fredholm index of the top horizontal arrow is index $\left(X_{\lambda_{0}}, X_{\lambda_{0}}^{\prime}, X_{\lambda_{0}}^{\prime \prime}, x_{0}\right)$, the index of the bottom horizontal arrow is index $\left(\Lambda \times X, X^{\prime}, X^{\prime \prime},\left(\lambda_{0}, x_{0}\right)\right)$, that of the left vertical arrow is $-2 \operatorname{dim} \Lambda$, and that of the right vertical arrow is $-\operatorname{dim} \Lambda$. (Here we have used the fact that the projections $X^{\prime} \rightarrow \Lambda$ and $X^{\prime \prime} \rightarrow \Lambda$ are submersions.) Hence assertion (i) follows from the fact that the Fredholm index of a composition is the sum of the Fredholm indices.

We prove (ii). Assume first that $h_{\lambda_{0}}$ is exact at $x_{0}$ and denote $y_{0}:=h_{\lambda_{0}}\left(x_{0}\right)$. We prove that the induced homomorphism

$$
d h\left(\lambda_{0}, x_{0}\right): T_{\left(\lambda_{0}, x_{0}\right)} X^{\prime} \cap T_{\left(\lambda_{0}, x_{0}\right)} X^{\prime \prime} \rightarrow T_{\left(\lambda_{0}, y_{0}\right)} Y^{\prime} \cap T_{\left(\lambda_{0}, y_{0}\right)} Y^{\prime \prime}
$$

is injective. If $(\hat{\lambda}, \hat{x}) \in T_{\left(\lambda_{0}, x_{0}\right)} X^{\prime} \cap T_{\left(\lambda_{0}, x_{0}\right)} X^{\prime \prime}$ and $d h\left(\lambda_{0}, x_{0}\right)(\hat{\lambda}, \hat{x})=0$ then

$$
\hat{\lambda}=0, \quad d h_{\lambda_{0}}\left(x_{0}\right) \hat{x}=0 .
$$

Since the projections $X^{\prime} \rightarrow \Lambda$ and $X^{\prime \prime} \rightarrow \Lambda$ are submersions we have $\hat{x} \in T_{x_{0}} X_{\lambda_{0}}^{\prime} \cap T_{x_{0}} X_{\lambda_{0}}^{\prime \prime}$. By assumption, this implies $\hat{x}=0$. This shows that (64) is injective, as claimed. We prove that the induced homomorphism

$$
d h\left(\lambda_{0}, x_{0}\right): \frac{T_{\left(\lambda_{0}, x_{0}\right)}(\Lambda \times X)}{T_{\left(\lambda_{0}, x_{0}\right)} X^{\prime}+T_{\left(\lambda_{0}, x_{0}\right)} X^{\prime \prime}} \rightarrow \frac{T_{\left(\lambda_{0}, y_{0}\right)}(\Lambda \times Y)}{T_{\left(\lambda_{0}, y_{0}\right)} Y^{\prime}+T_{\left(\lambda_{0}, y_{0}\right)} Y^{\prime \prime}}
$$


is surjective. Let $(\hat{\lambda}, \hat{y}) \in T_{\left(\lambda_{0}, y_{0}\right)}(\Lambda \times Y)$. Since the projection $X^{\prime} \rightarrow \Lambda$ is a submersion, there is a vector $\hat{x} \in T_{x_{0}} X$ such that $(\hat{\lambda}, \hat{x}) \in T_{\left(\lambda_{0}, x_{0}\right)} X^{\prime}$. Define $\hat{y}_{0} \in T_{y_{0}} Y$ by

$$
\left(0, \hat{y}_{0}\right):=(\hat{\lambda}, \hat{y})-d h\left(\lambda_{0}, x_{0}\right)(\hat{\lambda}, \hat{x}) .
$$

By assumption, there exists a vector $\hat{x}_{0} \in T_{x_{0}} X$ such that

$$
\hat{y}_{0}-d h_{\lambda_{0}}\left(x_{0}\right) \hat{x}_{0} \in T_{y_{0}} Y^{\prime}+T_{y_{0}} Y^{\prime \prime} \text {. }
$$

Hence

$$
\left(0, \hat{y}_{0}\right)-d h\left(\lambda_{0}, x_{0}\right)\left(0, \hat{x}_{0}\right) \in T_{\left(\lambda_{0}, y_{0}\right)} Y^{\prime}+T_{\left(\lambda_{0}, y_{0}\right)} Y^{\prime \prime}
$$

and hence

$$
(\hat{\lambda}, \hat{y})-d h\left(\lambda_{0}, x_{0}\right)\left(0, \hat{x}_{0}\right) \in T_{\left(\lambda_{0}, y_{0}\right)} Y^{\prime}+T_{\left(\lambda_{0}, y_{0}\right)} Y^{\prime \prime} .
$$

This shows that (65) is surjective, as claimed. Moreover, by (i) the quadruples $\left(\Lambda \times X, X^{\prime}, X^{\prime \prime}\right.$, $\left.\left(\lambda_{0}, x_{0}\right)\right)$ and $\left(\Lambda \times Y, Y^{\prime}, Y^{\prime \prime},\left(\lambda_{0}, y_{0}\right)\right)$ have the same Fredholm index. Hence (64) and (65) are bijective and so $h$ is exact at $\left(\lambda_{0}, x_{0}\right)$.

Conversely, assume that $h$ is exact at $\left(\lambda_{0}, x_{0}\right)$ so that (64) and (65) are bijective. We prove that the induced homomorphism

$$
d h_{\lambda_{0}}\left(x_{0}\right): T_{x_{0}} X_{\lambda_{0}}^{\prime} \cap T_{x_{0}} X_{\lambda_{0}}^{\prime \prime} \rightarrow T_{y_{0}} Y_{\lambda_{0}}^{\prime} \cap T_{y_{0}} Y_{\lambda_{0}}^{\prime \prime}
$$

is injective. Let $\hat{x} \in T_{x_{0}} X_{\lambda_{0}}^{\prime} \cap T_{x_{0}} X_{\lambda_{0}}^{\prime \prime}$ and suppose that $d h_{\lambda_{0}}\left(x_{0}\right) \hat{x}=0$. Then

$$
(0, \hat{x}) \in T_{\left(\lambda_{0}, x_{0}\right)} X^{\prime} \cap T_{\left(\lambda_{0}, x_{0}\right)} X^{\prime \prime}, \quad d h\left(\lambda_{0}, x_{0}\right)(0, \hat{x})=(0,0) .
$$

Since (64) is injective, this implies $\hat{x}=0$. This shows that (66) is injective. We prove that the induced homomorphism

$$
d h_{\lambda_{0}}\left(x_{0}\right): \frac{T_{x_{0}} X}{T_{x_{0}} X_{\lambda_{0}}^{\prime}+T_{x_{0}} X_{\lambda_{0}}^{\prime \prime}} \rightarrow \frac{T_{y_{0}} Y}{T_{y_{0}} Y_{\lambda_{0}}^{\prime}+T_{y_{0}} Y_{\lambda_{0}}^{\prime \prime}}
$$

is surjective. Let $\hat{y} \in T_{y_{0}} Y$. Since (65) is surjective, there exists a pair $(\hat{\lambda}, \hat{x}) \in T_{\left(\lambda_{0}, x_{0}\right)}(\Lambda \times X)$ such that

$$
(0, \hat{y})-d h\left(\lambda_{0}, x_{0}\right)(\hat{\lambda}, \hat{x}) \in T_{\left(\lambda_{0}, y_{0}\right)} Y^{\prime}+T_{\left(\lambda_{0}, y_{0}\right)} Y^{\prime \prime}
$$

Write

$$
(0, \hat{y})-d h\left(\lambda_{0}, x_{0}\right)(\hat{\lambda}, \hat{x})=\left(\hat{\lambda}^{\prime}, \hat{y}^{\prime}\right)+\left(\hat{\lambda}^{\prime \prime}, \hat{y}^{\prime \prime}\right)
$$

where

$$
\left(\hat{\lambda}^{\prime}, \hat{y}^{\prime}\right) \in T_{\left(\lambda_{0}, y_{0}\right)} Y^{\prime}, \quad\left(\hat{\lambda}^{\prime \prime}, \hat{y}^{\prime \prime}\right) \in T_{\left(\lambda_{0}, y_{0}\right)} Y^{\prime \prime} .
$$

Since the projections $X^{\prime} \rightarrow \Lambda$ and $X^{\prime \prime} \rightarrow \Lambda$ are submersions, there exist tangent vectors $\hat{x}^{\prime}, \hat{x}^{\prime \prime} \in T_{x_{0}} X$ such that

$$
\left(\hat{\lambda}^{\prime}, \hat{x}^{\prime}\right) \in T_{\left(\lambda_{0}, x_{0}\right)} X^{\prime}, \quad\left(\hat{\lambda}^{\prime \prime}, \hat{x}^{\prime \prime}\right) \in T_{\left(\lambda_{0}, x_{0}\right)} X^{\prime \prime} .
$$

Define the tangent vectors $\hat{y}_{0}^{\prime}, \hat{y}_{0}^{\prime \prime} \in T_{y_{0}} Y$ by

$$
\begin{aligned}
& \left(0, \hat{y}_{0}^{\prime}\right):=\left(\hat{\lambda}^{\prime}, \hat{y}^{\prime}\right)-d h\left(\lambda_{0}, x_{0}\right)\left(\hat{\lambda}^{\prime}, \hat{x}^{\prime}\right) \in T_{\left(\lambda_{0}, y_{0}\right)} Y^{\prime}, \\
& \left(0, \hat{y}_{0}^{\prime \prime}\right):=\left(\hat{\lambda}^{\prime \prime}, \hat{y}^{\prime \prime}\right)-d h\left(\lambda_{0}, x_{0}\right)\left(\hat{\lambda}^{\prime \prime}, \hat{x}^{\prime \prime}\right) \in T_{\left(\lambda_{0}, y_{0}\right)} Y^{\prime \prime} .
\end{aligned}
$$


Since the projections $Y^{\prime} \rightarrow \Lambda$ and $Y^{\prime \prime} \rightarrow \Lambda$ are submersions we have

$$
\hat{y}_{0}^{\prime} \in T_{y_{0}} Y_{\lambda_{0}}^{\prime}, \quad \hat{y}_{0}^{\prime \prime} \in T_{y_{0}} Y_{\lambda_{0}}^{\prime \prime} .
$$

Moreover, by (68), we have

$$
\hat{\lambda}+\hat{\lambda}^{\prime}+\hat{\lambda}^{\prime \prime}=0
$$

and hence, by (68) and (69),

$$
\hat{y}-d h_{\lambda_{0}}\left(x_{0}\right)\left(\hat{x}+\hat{x}^{\prime}+\hat{x}^{\prime \prime}\right)=\hat{y}_{0}^{\prime}+\hat{y}_{0}^{\prime \prime} \in T_{y_{0}} Y_{\lambda_{0}}^{\prime}+T_{y_{0}} Y_{\lambda_{0}}^{\prime \prime} .
$$

Hence (67) is surjective, as claimed. Now it follows again from the index identities in (i) that (66) and (67) are bijective and hence $h_{\lambda_{0}}$ is exact at $x_{0}$. This proves the theorem.

Corollary 6.9 Let $h_{\lambda}:\left(X, X_{\lambda}^{\prime}, X_{\lambda}^{\prime \prime}\right) \rightarrow\left(Y, Y_{\lambda}^{\prime}, Y_{\lambda}^{\prime \prime}\right)$ be as in Theorem 6.8 and suppose that $h_{\lambda_{0}}$ is exact at $x_{0} \in X_{\lambda_{0}}^{\prime} \cap X_{\lambda_{0}}^{\prime \prime}$. Then the following holds.

(i) If $\lambda$ is sufficiently close to $\lambda_{0}$ and $x \in X_{\lambda}^{\prime} \cap X_{\lambda}^{\prime \prime}$ is sufficiently close to $x_{0}$ then $h_{\lambda}$ is exact at $x$.

(ii) If $\Lambda \rightarrow Y: \lambda \mapsto y_{\lambda}$ is a smooth map such that $y_{\lambda} \in Y_{\lambda}^{\prime} \cap Y_{\lambda}^{\prime \prime}$ for every $\lambda$ then, after shrinking $\Lambda$ if necessary, there exists a unique smooth map $\Lambda \rightarrow X: \lambda \mapsto x_{\lambda}$ such that $x_{\lambda} \in X_{\lambda}^{\prime} \cap X_{\lambda}^{\prime \prime}$ and $h_{\lambda}\left(x_{\lambda}\right)=y_{\lambda}$ for every $\lambda$.

Proof Theorem 6.8 and Corollary 6.7

Remark 6.10 All the results of this section continue to hold in the complex category, i.e. all Hilbert spaces are complex, all Hilbert manifolds are complex, all maps are complex, the family $\left\{h_{\lambda}\right\}_{\lambda \in \Lambda}$ in Theorem 6.8 is a holomorphic family of holomorphic morphisms of complex Fredholm triples, etc. As a result the map $\Lambda \rightarrow X$ in Corollary 6.9 is holomorphic.

\section{Proofs of the main theorems}

Proof of Theorem 3.8. Assume the unfolding $\left(\pi_{B}: Q \rightarrow B, S_{*}, H_{B}, b_{0}\right)$ is iinfinitesimally universal. Let $\mathcal{U}, \mathcal{U}^{\prime}, \mathcal{U}^{\prime \prime}$ be the manifolds in 5.5 and let $\mathcal{V}, \mathcal{V}^{\prime}, \mathcal{V}^{\prime \prime}$ be the manifolds in 5.11 for

$$
P=Q, \quad A=B, \quad \pi_{A}=\pi_{B}, \quad R_{*}=S_{*}, \quad H_{A}=H_{B},
$$

and an appropriate Hardy decomposition $Q=Q^{\prime} \cup Q^{\prime \prime}$. For $a \in A=B$ denote $b_{a}:=a$, let $\alpha_{a}: \Gamma_{a} \rightarrow Q_{b_{a}}$ be the inclusion of $\Gamma_{a}:=Q_{a}^{\prime} \cap Q_{a}^{\prime \prime}$ into $Q_{b_{a}}$, and abbreviate $\beta_{a}:=$ $H_{B} \circ \alpha_{a}: \Gamma_{a} \rightarrow M$. Then the morphism

$$
\mathcal{U}_{a} \rightarrow \mathcal{V}_{a}:(\alpha, b) \mapsto \beta:=H_{B} \circ \alpha
$$

from the Fredholm quadruple $\left(\mathcal{U}_{a}, \mathcal{U}_{a}^{\prime}, \mathcal{U}_{a}^{\prime \prime},\left(\alpha_{a}, b_{a}\right)\right)$ to $\left(\mathcal{V}_{a}, \mathcal{V}_{a}^{\prime}, \mathcal{V}_{a}^{\prime \prime}, \beta_{a}\right)$ is exact for $a=$ $a_{0}=b_{0}$, by Theorems 5.9 and 5.12 (see 5.17). The same theorems assert that the family (70) of morphisms of Fredholm quadruples satisfies the requirements of Theorem 6.8. Hence it follows from Corollary 6.9 that (70) is exact for $a=b$ sufficiently close to $a_{0}=b_{0}$. Hence, again by Theorems 5.9 and 5.12 , the unfolding $\left(\pi_{B}: Q \rightarrow B, S_{*}, H_{B}, b\right)$ is infinitesimally universal for $b$ sufficiently close to $b_{0}$. This proves the theorem. 
Proof of Theorem 3.6. We proved 'only if' in Sect. 3. To prove 'if' assume that $\left(\pi_{B}: Q \rightarrow\right.$ $\left.B, S_{*}, H_{B}, b_{0}\right)$ is an infinitesimally universal unfolding. We prove that it is universal. Let $\left(\pi_{A}: P \rightarrow A, R_{*}, H_{A}, a_{0}\right)$ be another unfolding of maps and $f_{0}: P_{a_{0}} \rightarrow Q_{b_{0}}$ be a fiber isomorphism. Choose a Hardy decomposition $P=P^{\prime} \cup P^{\prime \prime}$ and open subsets $U^{\prime}, U^{\prime \prime}$, and $U:=U^{\prime} \cap U^{\prime \prime}$ of $Q$ as in 5.2, 5.3, and 5.4. Let $\mathcal{U}, \mathcal{U}^{\prime}, \mathcal{U}^{\prime \prime}$ be as in 5.5 and $\mathcal{V}, \mathcal{V}^{\prime}, \mathcal{V}^{\prime \prime}$ be as in 5.11. Then

$$
\left(\alpha_{0}:=f_{0} \mid \Gamma_{a_{0}}, b_{0}\right) \in \mathcal{U}_{a_{0}}^{\prime} \cap \mathcal{U}_{a_{0}}^{\prime \prime}, \quad \beta_{0}:=H_{A} \mid \Gamma_{a_{0}} \in \mathcal{V}_{a_{0}}^{\prime} \cap \mathcal{V}_{a_{0}}^{\prime \prime} .
$$

Since the unfolding $\left(\pi_{B}, S_{*}, H_{B}, b_{0}\right)$ is infintesimally universal the map

$$
\mathcal{U}_{a_{0}} \rightarrow \mathcal{V}_{a_{0}}:(\alpha, b) \mapsto \beta:=H_{B} \circ \alpha
$$

is an exact morphism of Fredholm triples as in 6.5 (see 5.17). By Theorems 5.9 and 5.12 the family of maps

$$
\mathcal{U}_{a} \rightarrow \mathcal{V}_{a}:(\alpha, b) \mapsto \beta:=H_{B} \circ \alpha,
$$

parametrized by $a \in A$ satisfies the hypotheses of Theorem 6.8 (in the complex category). Moreover, there is a holomorphic map

$$
A \rightarrow \mathcal{V}: a \mapsto\left(a, \beta_{a}\right), \quad \beta_{a}:=H_{A} \mid \Gamma_{a} \in \mathcal{V}_{a}^{\prime} \cap \mathcal{V}_{a}^{\prime \prime}
$$

Hence it follows from Corollary 6.9 and Remark 6.10 that, after shrinking $A$ if necessary, there exists a unique holomorphic map

$$
A \rightarrow \mathcal{U}: a \mapsto\left(a, \alpha_{a}, b_{a}\right), \quad\left(\alpha_{a}, b_{a}\right) \in \mathcal{U}_{a}^{\prime} \cap \mathcal{U}_{a}^{\prime \prime},
$$

such that $\beta_{a}=H_{B} \circ \alpha_{a}$ for every $a \in A$. Define $\phi: A \rightarrow B$ by $\phi(a):=b_{a}$, for every $a \in A$ let $f_{a}: P_{a} \rightarrow Q_{b_{a}}$ be the unique fiber isomorphism with $f_{a} \mid \Gamma_{a}=\alpha_{a}$, and define $\Phi: P \rightarrow Q$ by $\Phi \mid P_{a}:=f_{a}$. Then $\phi$ is holomorphic. That the restriction of $\Phi$ to $\operatorname{int}\left(P^{\prime}\right)$ is holomorphic follows from [4, Lemma 10.18]. To prove that the restriction of $\Phi$ to int $\left(P^{\prime \prime}\right)$ is holomorphic we write it as the composition

$$
\operatorname{int}\left(P^{\prime \prime}\right) \rightarrow A \times \Omega \rightarrow \mathcal{U}^{\prime \prime} \times \Omega \rightarrow Q
$$

where the first map is $\pi_{A} \times \rho$, the second map is the product of (71) with the identity, and the third map is the evaluation map $\left(a, f^{\prime \prime}, z\right) \mapsto f^{\prime \prime}\left(\rho_{a}^{-1}(z)\right)$. All four spaces are complex manifolds and all three maps are holomorphic. The argument is as in Step 3 in the proof of [4, Theorem 5.3]. It is important to remember that the complex structure on the factor $\Omega$ depends on $a \in A$ and is twisted by $\eta(a, \hat{a})$ as in (14). This proves that $\Phi$ is holomorphic on $P \backslash \partial P^{\prime}$. Since $\Phi$ is continuous, it is holomorphic everywhere. This proves the theorem.

Proof of Theorem 3.5. Given the work done in Sect. 3 it remains to prove 'if' under the assumptions that $\left(\Sigma, s_{0, *}, v_{0}, j_{0}, v_{0}\right)$ is a regular stable map and the underlying marked nodal Riemann surface $\left(\Sigma, s_{0, *}, v_{0}, j_{0}\right)$ is still stable. Let $\left(\pi_{A}: P \rightarrow A, R_{*}, a_{0}\right)$ be a universal unfolding of this marked nodal Riemann surface (in the sense of [4, Definition 5.1]) and $w_{0}: \Sigma \rightarrow P_{a_{0}}$ be a desingularization of the central fiber. Define the holomorphic map $h_{0}: P_{a_{0}} \rightarrow M$ by $h_{0} \circ w_{0}:=v_{0}$. Choose a Hardy decomposition

$$
P=P^{\prime} \cup P^{\prime \prime}, \quad \Gamma_{a}:=P_{a} \cap P^{\prime} \cap P^{\prime \prime},
$$

as in 5.3, fix an integer $s+1 / 2>1$, and define $\mathcal{V}, \mathcal{V}^{\prime}, \mathcal{V}^{\prime \prime}$ as in 5.11. The desingularization $w_{0}: \Sigma \rightarrow P_{a_{0}}$ induces a decomposition

$$
\Sigma=\Sigma^{\prime} \cup \Sigma^{\prime \prime}, \quad \Sigma^{\prime} \cap \Sigma^{\prime \prime}=\partial \Sigma^{\prime}=\partial \Sigma^{\prime \prime},
$$


with $\Sigma^{\prime}:=w_{0}^{-1}\left(P^{\prime}\right)$ and $\Sigma^{\prime \prime}:=w_{0}^{-1}\left(P^{\prime \prime}\right)$. As in the proof of Theorem 5.12 the map $w_{0}^{-1} \circ \iota_{a_{0}}$ is a diffeomorphism from $\Gamma$ in (26) to $\Sigma^{\prime} \cap \Sigma^{\prime \prime}$ and, to simplify the notation, we assume that $\Gamma=\Sigma^{\prime} \cap \Sigma^{\prime \prime}$ so that $\iota_{a_{0}}=w_{0} \mid \Gamma: \Gamma \rightarrow P_{a_{0}}$. The infinitesimally universal unfolding of the stable map $\left(\Sigma, s_{0, *}, v_{0}, j_{0}, v_{0}\right)$ is the tuple

$$
\left(\pi_{B}: Q \rightarrow B, S_{*}, H_{B}, b_{0}\right)
$$

defined by

$$
\begin{gathered}
B:=\mathcal{V}^{\prime} \cap \mathcal{V}^{\prime \prime}, \quad Q:=\left\{(p, \beta) \in P \times B \mid \beta \in \mathcal{V}_{\pi_{A}(p)}^{\prime} \cap \mathcal{V}_{\pi_{A}(p)}^{\prime \prime}\right\}, \\
\pi_{B}(p, \beta):=\left(\pi_{A}(p), \beta\right), \quad b_{0}:=\left(a_{0}, \beta_{0}\right), \\
S_{\mathrm{i}}:=\left\{(p, \beta) \in Q \mid p \in R_{\mathrm{i}}\right\}, \quad H_{B}(p, \beta):=h_{\beta}(p),
\end{gathered}
$$

where $h_{\beta}: P_{a} \rightarrow M$ is the unique holomorphic map with

$$
h_{\beta} \mid \Gamma_{a}=\beta .
$$

As in 5.11, $\mathcal{V}$ is a complex Hilbert manifold and by part (iii) of Theorem 5.12 the sets $\mathcal{V}^{\prime}$ and $\mathcal{V}^{\prime \prime}$ are complex submanifolds of $\mathcal{V}$. By part (i) of Theorem 5.16, the submanifolds $\mathcal{V}^{\prime}$ and $\mathcal{V}^{\prime \prime}$ intersect transversally at $\left(a_{0}, \beta_{0}\right)$ and hence $B=\mathcal{V}^{\prime} \cap \mathcal{V}^{\prime \prime}$ is a complex submanifold of $\mathcal{V}$ (after shrinking $\mathcal{V}^{\prime}$ and $\mathcal{V}^{\prime \prime}$ if necessary). By Theorem 5.12, $B$ has dimension

$$
\operatorname{dim}_{\mathbb{C}} B=(\mathrm{m}-3)(1-\mathrm{g})+\left\langle c_{1}, \mathrm{~d}\right\rangle+\mathrm{n} .
$$

We prove that $Q$ is a complex submanifold of $P \times \mathcal{V}$. Define

$$
f: B \rightarrow A \text { by } f(a, \beta):=a
$$

for $(a, \beta) \in B=\mathcal{V}^{\prime} \cap \mathcal{V}^{\prime \prime}$. Then the projection $\pi_{B}: Q \rightarrow B$ is the pullback of the projection $\pi_{A}: P \rightarrow A$ by the map $f$, i.e. $Q$ is the preimage of the diagonal in $A \times A$ under the holomorphic map

$$
\pi_{A} \times f: P \times B \rightarrow A \times A
$$

and $\pi_{B}$ is the restriction of projection on the first factor to $Q$. The map $\pi_{A} \times f$ is transverse to the diagonal if and only if

$$
T_{\pi_{A}(p)} A=\operatorname{im} d \pi_{A}(p)+d \pi_{\mathcal{V}}(a, \beta)\left(T_{(a, \beta)} \mathcal{V}^{\prime} \cap T_{(a, \beta)} \mathcal{V}^{\prime \prime}\right)
$$

for every $p \in P$ and every $\beta \in \mathcal{V}_{a}^{\prime} \cap \mathcal{V}_{a}^{\prime \prime}$ with $a=\pi_{A}(p)$, where $\pi_{\mathcal{V}}: \mathcal{V} \rightarrow A$ denotes the obvious projection. Equation (74) follows immediately from part (ii) of Theorem 5.16. Hence $Q$ is a complex submanifold of $P \times \mathcal{V}$ and the projection $\pi_{B}: Q \rightarrow B$ is holomorphic. We prove that the map $\pi_{B}$ is a nodal family of Riemann surfaces in Lemma 7.1 below. The subset $S_{\mathrm{i}} \subset Q$ is the transverse intersection of the complex submanifolds $R_{\mathrm{i}} \times \mathcal{V}$ and $Q$, and hence is a complex submanifold of $Q$ (of codimension one).

We prove that $H_{B}: Q \rightarrow M$ is holomorphic. For this we use the Hardy decomposition

$$
Q=Q^{\prime} \cup Q^{\prime \prime}, \quad Q^{\prime}:=Q \cap\left(P^{\prime} \times \mathcal{V}\right), \quad Q^{\prime \prime}:=Q \cap\left(P^{\prime \prime} \times \mathcal{V}\right) .
$$

That $H_{B}$ is holomorphic in the interior of $Q^{\prime}$ follows from Lemma 5.14 (iii). To prove that $H_{B}$ is holomorphic in the interior of $Q^{\prime \prime}$ write it as the composition

$$
\operatorname{int}\left(Q^{\prime \prime}\right) \rightarrow B \times \Omega \rightarrow \mathcal{V}^{\prime \prime} \times \Omega \rightarrow M
$$


where the first map is given by a Hardy trivialization $\pi_{B} \times \rho$, the second by the inclusion $B \rightarrow \mathcal{V}^{\prime \prime}$, and the third is the evaluation map $((a, \beta), z) \mapsto\left(h_{\beta}^{\prime \prime}\left(\rho_{a}^{-1}(z)\right)\right)$ where $h_{\beta}^{\prime \prime}: P_{a}^{\prime \prime} \rightarrow$ $M$ is the unique holomorphic map with $h_{\beta}^{\prime \prime} \mid \Gamma_{a}=\beta$. As in the proof of Theorem 3.6 all four spaces are complex manifolds and all three maps are holomorphic. This proves that $H_{B}$ is holomorphic in $Q \backslash \partial Q^{\prime}$. Since $H_{B}$ is continuous it is holomorphic everywhere.

We prove that the unfolding $\left(\pi_{B}: Q \rightarrow B, S_{*}, H_{B}, b_{0}\right)$ is infinitesimally universal. Note that $Q_{b_{0}}=P_{a_{0}} \times\left\{\beta_{0}\right\}$ and define $u_{0}: \Sigma \rightarrow Q_{b_{0}}$ by

$$
u_{0}(z):=\left(w_{0}(z), \beta_{0}\right)
$$

Since $h_{\beta_{0}} \circ w_{0}=v_{0}$ we have

$$
H_{B} \circ u_{0}(z)=H_{B}\left(w_{0}(z), \beta_{0}\right)=h_{\beta_{0}}\left(w_{0}(z)\right)=v_{0}(z)
$$

for every $z \in \Sigma$. As before we denote by $f: B=\mathcal{V}^{\prime} \cap \mathcal{V}^{\prime \prime} \rightarrow A$ the obvious projection and by $b_{0}=\left(a_{0}, \beta_{0}\right) \in B$ the base point. Then the kernel of the derivative $d f\left(b_{0}\right): T_{b_{0}} B \rightarrow$ $T_{a_{0}} A$ is the intersection $T_{\beta} \mathcal{V}_{a_{0}}^{\prime} \cap T_{\beta} \mathcal{V}_{a_{0}}^{\prime \prime}$. Hence, for $z \in \Sigma$ we have $p:=w_{0}(z) \in P_{a_{0}}$, $q:=u_{0}(z)=\left(w_{0}(z), \beta_{0}\right) \in Q_{b_{0}}$, and

$$
\operatorname{ker} d\left(f \circ \pi_{B}\right)(q)=\operatorname{ker} d \pi_{A}(p) \times\left(T_{\beta} \mathcal{V}_{a_{0}}^{\prime} \cap T_{\beta} \mathcal{V}_{a_{0}}^{\prime \prime}\right) .
$$

The restriction of $d H_{B}(q): T_{q} Q \rightarrow T_{v_{0}(z)} M$ to this space is

$$
d H_{B}\left(u_{0}(z)\right)(\hat{p}, \hat{\beta})=\hat{v}(z)+d v_{0}(z) \hat{z}
$$

where $\hat{z} \in T_{z} \Sigma$ is the unique element with $d w_{0}(z) \hat{z}=\hat{p}$ and $\hat{v} \in \Omega^{0}\left(\Sigma / v, v_{0}^{*} T M\right)$ is the unique vector field along $v_{0}$ that satisfies the nodal condition, belongs to the kernel of $D_{v_{0}}$, and satisfies $\hat{v} \mid \Gamma=\hat{\beta} \circ \iota_{a_{0}}$.

We prove that the induced map

$$
d H_{B}\left(u_{0}\right): \operatorname{ker} D_{u_{0}} \rightarrow \operatorname{ker} D_{v_{0}}
$$

is bijective. The domain of $\mathcal{D}_{u_{0}}$ is the space

$$
\mathcal{X}_{u_{0}}:=\left\{\begin{array}{l|l}
(\hat{w}, \hat{b}) \in \Omega^{0}\left(\Sigma / \nu, w_{0}^{*} T P\right) \times T_{b} B & \begin{array}{l}
\hat{w}\left(s_{0, \mathrm{i}}\right) \in T_{w_{0}\left(s_{0, \mathrm{i}}\right)} R_{\mathrm{i}} \\
d \pi_{A}\left(w_{0}\right) \hat{w} \equiv d f\left(b_{0}\right) \hat{b}
\end{array}
\end{array}\right\},
$$

the target space can be identified with

$$
\mathcal{Y}_{u_{0}}=\mathcal{Y}_{w_{0}}=\left\{\eta \in \Omega^{0,1}\left(\Sigma, w_{0}^{*} T P\right) \mid d \pi_{A}\left(w_{0}\right) \eta \equiv 0\right\},
$$

and the operator is given by

$$
D_{u_{0}}(\hat{w}, \hat{b}):=D_{w_{0}} \hat{w} .
$$

Since the unfolding $\left(\pi_{A}, R_{*}, a_{0}\right)$ (of marked nodal Riemann surfaces) is universal, the operator

$$
D_{w_{0}}: \mathcal{X}_{w_{0}}:=\left\{\begin{array}{l|l}
\hat{w} \in \Omega^{0}\left(\Sigma / \nu, w_{0}^{*} T P\right) & \begin{array}{l}
\hat{w}\left(s_{0, \mathrm{i}}\right) \in T_{w_{0}\left(s_{0, \mathrm{i}}\right)} R_{\mathrm{i}} \\
d \pi_{A}\left(w_{0}\right) \hat{w} \equiv \text { constant }
\end{array}
\end{array}\right\} \rightarrow \mathcal{Y}_{u_{0}}
$$

is bijective. It follows that the projection $(\hat{w}, \hat{b}) \mapsto \hat{b}$ is an isomorphism from the kernel of $D_{u_{0}}$ to the kernel of the linear map $d f\left(b_{0}\right): T_{b_{0}} B \rightarrow T_{a_{0}} A$. Now recall that $f: B=\mathcal{V}^{\prime} \cap \mathcal{V}^{\prime \prime} \rightarrow A$ denotes the obvious projection. Then the kernel of $d f\left(a_{0}, \beta_{0}\right): T_{\left(a_{0}, \beta_{0}\right)}\left(\mathcal{V}^{\prime} \cap \mathcal{V}^{\prime \prime}\right) \rightarrow T_{a_{0}} A$ 
is the intersection $T_{\beta_{0}} \mathcal{V}_{a_{0}}^{\prime} \cap T_{\beta_{0}} \mathcal{V}_{a_{0}}^{\prime}$ which, by Theorem 5.12 (ii), is isomorphic to the kernel of $D_{v_{0}}$. The composite isomorphism

$$
\operatorname{ker} D_{u_{0}} \rightarrow \operatorname{ker} d f\left(a_{0}, \beta_{0}\right) \rightarrow \operatorname{ker} D_{v_{0}}
$$

is given by $(0, \hat{b}) \mapsto \hat{\beta} \mapsto \hat{v}$ where $\hat{b}=(0, \hat{\beta})$ and $\hat{v}$ is the unique element in the kernel of $D_{v_{0}}$ with $\hat{v} \mid \Gamma=\hat{\beta} \circ \iota_{a_{0}}$. This map is precisely (75) which is therefore an isomorphism.

Now it follows from Theorem 5.16 (ii) that the nodal family $\left(\pi_{B}, S_{*}, b_{0}\right)$ is regular nodal, i.e. the projections of the critical manifolds intersect transversally at $b_{0}$. Hence, by [4, Lemma 12.2], the operator $D_{u_{0}}$ has Fredholm index

$$
\begin{aligned}
\operatorname{index}_{\mathbb{C}}\left(D_{u_{0}}\right) & =3-3 \mathrm{~g}-\mathrm{n}+\operatorname{dim}_{\mathbb{C}} B \\
& =\mathrm{m}(1-\mathrm{g})+\left\langle c_{1}, \mathrm{~d}\right\rangle \\
& =\operatorname{index}_{\mathbb{C}}\left(D_{v_{0}}\right) .
\end{aligned}
$$

Here the second equality follows from (73). Since the kernels are isomorphic it follows that cokernels of $D_{u_{0}}$ and $D_{v_{0}}$ have the same dimensions. Moreover, the induced homomorphism $d H_{B}\left(u_{0}\right): \operatorname{coker} D_{u_{0}} \rightarrow$ coker $D_{v_{0}}$ is surjective, by Remark 3.4, and hence is bijective. This completes the proof of Theorem 3.5.

Lemma 7.1 Let $\pi_{A}: P \rightarrow A$ be a nodal family and $f: B \rightarrow A$ be a holomorphic map such that $f \times \pi_{A}: B \times P \rightarrow A \times A$ is transverse to the diagonal. Then the pullback $\pi_{B}: Q \rightarrow B$ of $\pi_{A}$ by $f$ is a nodal family.

Proof The pullback is defined by

$$
Q:=\left\{(b, p) \in B \times P \mid \pi_{A}(p)=f(b)\right\}, \quad \pi_{B}(b, p):=b .
$$

The condition that $f \times \pi_{A}: B \times P \rightarrow A \times A$ is transverse to the diagonal implies that $Q$ is a submanifold of $B \times P$. We prove that

(i) $(b, p) \in Q$ is a regular point of $\pi_{B}$ if $p$ is a regular point of $\pi_{A}$, and

(ii) $(b, p) \in Q$ is a nodal point of $\pi_{B}$ if $p$ is a nodal point of $\pi_{A}$.

To prove (i) assume w.l.o.g. that $P=\mathbb{C} \times A$ so $Q=\mathbb{C} \times \operatorname{graph}(f)$. Then $\pi_{B}(b, z, f(b))=b$ so $\pi_{B}$ is a submersion.

To prove (ii) assume that w.l.o.g. that $P=\mathbb{C} \times \mathbb{C} \times U, A=\mathbb{C} \times U, \pi_{A}(x, y, u)=(x y, u)$, and $f(b)=(\zeta(b), g(b)) \in \mathbb{C} \times U$. Then

$$
Q=\{(b, x, y, u) \mid x y=z=\zeta(b), \quad u=g(b)\} .
$$

The condition that $f \times \pi_{A}$ is transverse to the diagonal at $(b, x, y, u) \in Q$ is that for all $\left(\hat{z}_{1}, \hat{u}_{1}, \hat{z}_{2}, \hat{u}_{2}\right) \in T_{(z, u)} A \times T_{(z, u)} A=\mathbb{C} \times T_{u} U \times \mathbb{C} \times T_{u} U$ the equations

$$
\begin{gathered}
\hat{z}_{1}=d \zeta(b) \hat{b}+\hat{z} \\
\hat{u}_{1}=d g(b) \hat{b}+\hat{u} \\
\hat{z}_{2}=\hat{x} y+x \hat{y}+\hat{z} \\
\hat{u}_{2}=\hat{v}+\hat{u}
\end{gathered}
$$

have a solution

$$
\hat{b} \in T_{b} B, \quad(\hat{x}, \hat{y}, \hat{v}) \in T_{(x, y, u)} P=\mathbb{C}^{2} \times T_{u} U, \quad(\hat{z}, \hat{u}) \in T_{a} A=\mathbb{C} \times T_{u} U .
$$

At a nodal point we have $x=y=0$ so transversality implies that $d \zeta(b) \neq 0$. This implies that there is a coordinate system on $B$ with $\zeta$ as its first element. The pullback to $Q$ of the 
coordinates other than $\zeta$ together with the functions $x$ and $y$ give the desired nodal coordinates on $Q$. This proves (ii) and the lemma.

Corollary 7.2 Let $\pi_{A}: P \rightarrow A$ be regular nodal family and $f: B \rightarrow A$ be a holomorphic map which is transverse to the core $A_{0}$ of $\pi_{A}$. Then the hypothesis of Lemma 7.1 holds, the pullback $\pi_{B}: Q \rightarrow B$ is regular nodal, and its core is $B_{0}:=f^{-1}\left(A_{0}\right)$.

Proof Denote by $C_{1}, \ldots, C_{\mathrm{k}} \subset P$ the components of the singular set of $\pi_{A}$. The proof of Lemma 7.1 shows that the hypothesis that $f \times \pi_{A}$ is transverse to the diagonal is equivalent to the hypothesis that $f$ is transverse to each $\pi_{A}\left(C_{\mathrm{i}}\right)$. The hypothesis that $\pi_{A}$ is regular nodal is that these projections $\pi_{A}\left(C_{\mathrm{i}}\right)$ of the critical manifolds intersect transversally. Hence $T_{a} A_{0}=\bigcap_{\mathrm{i}} T_{a} \pi_{A}\left(C_{\mathrm{i}}\right)$ so $f$ is certainly transverse to each $\pi_{A}\left(C_{\mathrm{i}}\right)$ and the hypothesis of Lemma 7.1 holds.

The hypothesis that $\pi_{A}$ is regular nodal implies that in a neighborhood of each point of the core $A_{0}$ of $\pi_{A}$ there are coordinates $z_{1}, \ldots, z_{\mathrm{k}}, u_{1}, \ldots$ on $A$ such that for each $\mathrm{i}, z_{i}$ together with the remaining coordinates for the base coordinates of a nodal coordinate system. In particular, $\pi_{A}\left(C_{\mathrm{i}}\right)=\left\{z_{\mathrm{i}}=0\right\}$. The transversality hypothesis implies that the functions $f^{*} z_{\mathrm{i}}$ are independent, i.e. the sequence $f^{*} z_{1}, \ldots, f^{*} z_{\mathrm{k}}$ extends to a coordinate system on $B$. Now the proof of Lemma 7.1 shows that for each $\mathrm{i}$ a reordering of these coordinates which puts $f^{*} z_{\mathrm{i}}$ first is the base coordinate system of a nodal coordinate system. The core $B_{0}$ is then defined by $f^{*} z_{1}=\cdots f^{*} z_{\mathrm{k}}=0$ which shows that $B_{0}=f^{-1}\left(A_{0}\right)$.

Definition 7.3 Let $\left(\pi_{A}: P \rightarrow A, R_{*}, H_{A}, a_{0}\right)$ and $\left(\pi_{B}: Q \rightarrow B, S_{*}, H_{B}, b_{0}\right)$ be two unfoldings of type $(\mathrm{g}, \mathrm{n}, \mathrm{d})$. A sequence of fiber isomorphisms $f_{k}: P_{a_{k}} \rightarrow Q_{b_{k}}$ is said to DMG converge to a fiber isomorphism $f_{0}: P_{a_{0}} \rightarrow Q_{b_{0}}$ if $a_{k} \rightarrow a_{0}, b_{k} \rightarrow b_{0}$, and for every Hardy decomposition $P=P^{\prime} \cup P^{\prime \prime}$ as in 5.3 the sequence $f_{k} \circ \iota_{a_{k}}: \Gamma \rightarrow Q$ converges to $f_{0} \circ \iota_{a_{0}}: \Gamma \rightarrow Q$ in the $C^{\infty}$ topology. (DMG convergence of fiber isomorphisms is essentially the same as DM convergence in [4, Definition 13.7]. The only difference is that in the former case we deal with unfoldings of stable maps whereas in the latter case we deal with unfoldings of marked nodal Riemann surfaces, i.e. the two notions of fiber isomorphism differ.)

Lemma 7.4 Let $\left(\pi_{A}: P \rightarrow A, R_{*}, H_{A}, a_{0}\right)$ and $\left(\pi_{B}: Q \rightarrow B, S_{*}, H_{B}, b_{0}\right)$ be two universal unfoldings of type $(\mathrm{g}, \mathrm{n}, \mathrm{d}),(\Phi, \phi):(P, A) \rightarrow(Q, B)$ be the germ of a morphism satisfying $H_{B} \circ \Phi=H_{A}, \phi\left(a_{0}\right)=b_{0}$, and $\Phi_{a_{0}}=f_{0}, a_{k} \in A$ and $b_{k} \in B$ be two sequences with $a_{k} \rightarrow a_{0}$ and $b_{k} \rightarrow b_{0}$, and $f_{k}: P_{a_{k}} \rightarrow Q_{b_{k}}$ be a sequence of fiber isomorphisms. Then the following are equivalent.

(i) The sequence $\left(a_{k}, f_{k}, b_{k}\right)$ DMG converges to $\left(a_{0}, f_{0}, b_{0}\right)$.

(ii) For $k$ sufficiently large we have $\phi\left(a_{k}\right)=b_{k}$ and $\Phi_{a_{k}}=f_{k}$.

Proof That (ii) implies (i) is obvious. We prove that (i) implies (ii). Recall the Hardy decomposition in the definition of the spaces $\mathcal{U}, \mathcal{U}^{\prime}, \mathcal{U}^{\prime \prime}$ in 5.5 and $\mathcal{V}, \mathcal{V}^{\prime}, \mathcal{V}^{\prime \prime}$ in 5.11. Then

$$
\left(a, \Phi_{a} \mid \Gamma_{a}, \phi(a)\right) \in \mathcal{U}^{\prime} \cap \mathcal{U}^{\prime \prime}, \quad\left(a_{k}, f_{k} \mid \Gamma_{a_{k}}, b_{k}\right) \in \mathcal{U}^{\prime} \cap \mathcal{U}^{\prime \prime}
$$

for every $a \in A$ and every sufficiently large $k$, by DMG convergence. The sequences $\left(a_{k}, \Phi_{a_{k}} \mid \Gamma_{a_{k}}, \phi\left(a_{k}\right)\right)$ and $\left(a_{k}, f_{k} \mid \Gamma_{a_{k}}, b_{k}\right)$ converge to the same point $\left(a_{0}, f_{0} \mid \Gamma_{a_{0}}, b_{0}\right) \in$ $\mathcal{U}^{\prime} \cap \mathcal{U}^{\prime \prime}$. Moreover, their images under the Fredholm map

$$
\mathcal{U}^{\prime} \cap \mathcal{U}^{\prime \prime} \rightarrow \mathcal{V}^{\prime} \cap \mathcal{V}^{\prime \prime}:(a, \alpha, b) \mapsto\left(a, H_{B} \circ \alpha\right)
$$


agree because

$$
H_{B} \circ f_{k}=H_{A} \mid P_{a_{k}}=H_{B} \circ \Phi_{a_{k}} .
$$

Moreover it follows from infinitesimal universality and Theorems 5.9, 5.12, and 6.8 that the map $(a, \alpha, b) \mapsto\left(a, H_{B} \circ \alpha\right)$ from $\left(\mathcal{U}, \mathcal{U}^{\prime}, \mathcal{U}^{\prime \prime},\left(a_{0}, f_{0} \mid \Gamma_{0}, b_{0}\right)\right)$ to $\left(\mathcal{V}, \mathcal{V}^{\prime}, \mathcal{V}^{\prime \prime},\left(a_{0}, H_{B}\right.\right.$ 。 $\left.f_{0} \mid \Gamma_{0}\right)$ ) is an exact morphism of Fredholm quadruples (see 6.5). Hence $\left(f_{k} \mid \Gamma_{a_{k}}, b_{k}\right)=$ $\left(\Phi_{a_{k}} \mid \Gamma_{a_{k}}, \phi\left(a_{k}\right)\right)$ for $k$ sufficiently large, by Corollary 6.7, and hence also $f_{k}=\Phi_{a_{k}}$. This proves the lemma.

Proof of Theorem 3.11. Let $\left(\pi: Q \rightarrow B, S_{*}, H\right.$ ) be a universal family and denote by $(B, \Gamma)$ the associated etale groupoid of 3.10. We prove that this groupoid is proper. Thus let $\left(a_{k}, f_{k}, b_{k}\right)$ be a sequence in $\Gamma$ such that $a_{k}$ converges to $a_{0}$ and $b_{k}$ converges to $b_{0}$. We must show that there is a fiber isomorphism $f_{0}: Q_{a_{0}} \rightarrow Q_{b_{0}}$ such that a suitable subsequence of $f_{k}$ DMG converges to $f_{0}$. To see this we assume first that the underlying marked nodal Riemann surface associated to a desingularization of $Q_{a_{0}}$ is stable. Then the same holds for $Q_{b_{0}}$ and we may assume w.l.o.g. that our universal unfolding has the form (72) as constructed in the proof of Theorem 3.5 near $a_{0}$ and $b_{0}$. It then follows that $\left(a_{k}, f_{k}, b_{k}\right)$ induces a sequence $\left(a_{k}^{\prime}, f_{k}^{\prime}, b_{k}^{\prime}\right)$ of fiber isomorphisms for the underlying universal family $\left(\pi^{\prime}: Q^{\prime} \rightarrow B^{\prime}, S_{*}^{\prime}\right)$ of stable marked nodal Riemann surfaces such that $a_{k}^{\prime}$ and $b_{k}^{\prime}$ converge to $a_{0}^{\prime}$ and $b_{0}^{\prime}$, respectively. By [4, Theorem 6.6], the sequence $f_{k}^{\prime}$ DM-converges to a fiber isomorphism $f_{0}^{\prime}: Q_{a_{0}^{\prime}}^{\prime} \rightarrow Q_{b_{0}^{\prime}}^{\prime}$. Since $H_{B} \circ f_{k}=H_{B} \mid Q_{a_{k}}$, we find that $f_{0}^{\prime}$ induces a fiber isomorphism $f_{0}: Q_{a_{0}} \rightarrow Q_{b_{0}}$ and it follows from the definitions that $f_{k}$ DMG converges to $f_{0}$. This proves the assertion under the stability assumption for the underlying marked nodal Riemann surface. If that does not hold, we choose an embedding of our universal family into another family $\left(\pi^{\prime}: Q^{\prime} \rightarrow B^{\prime}, S_{*}^{\prime}, T_{*}^{\prime}, H^{\prime}\right.$ ) that is a universal unfolding of each of its fibers and remains stable after discarding $H^{\prime}$. Then the existence of a DMG-convergent subsequence follows immediately from what we have already proved.

\section{The Gromov topology}

In this section we prove that the topology on the moduli space of (regular) stable maps that is induced by the orbifold structure agrees with the topology used elsewhere in the literature. To define convergence of a sequence in this topology we need to recall the notion of deformation from [4, Definition 13.2] (Fig. 2).

8.1 Let $\Sigma$ be a compact oriented surface and $\gamma \subset \Sigma$ be a disjoint union of embedded circles. We denote by $\Sigma_{\gamma}$ the compact surface with boundary which results by cutting open $\Sigma$ along $\gamma$. This implies that there is a local embedding

$$
\sigma: \Sigma_{\gamma} \rightarrow \Sigma
$$

which maps int $\left(\Sigma_{\gamma}\right)$ one to one onto $\Sigma \backslash \gamma$ and maps $\partial \Sigma_{\gamma}$ two to one onto $\gamma$. One might call $\sigma$ the suture map and $\gamma$ the incision.

Definition 8.2 Let $\left(\Sigma^{\prime}, v^{\prime}\right)$ and $(\Sigma, v)$ be nodal surfaces. A smooth map $\phi: \Sigma^{\prime} \backslash \gamma^{\prime} \rightarrow \Sigma$ is called a $\left(v^{\prime}, v\right)$-deformation iff $\gamma^{\prime} \subset \Sigma^{\prime} \backslash \bigcup v^{\prime}$ is a disjoint union of embedded circles such that (where $\sigma: \Sigma_{\gamma^{\prime}}^{\prime} \rightarrow \Sigma^{\prime}$ is the suture map just defined) we have

- $\phi_{*} v^{\prime}:=\left\{\left\{\phi\left(y_{1}^{\prime}\right), \phi\left(y_{2}^{\prime}\right)\right\} \mid\left\{y_{1}^{\prime}, y_{2}^{\prime}\right\} \in v^{\prime}\right\} \subset v$. 
Fig. 2 Gromov convergence

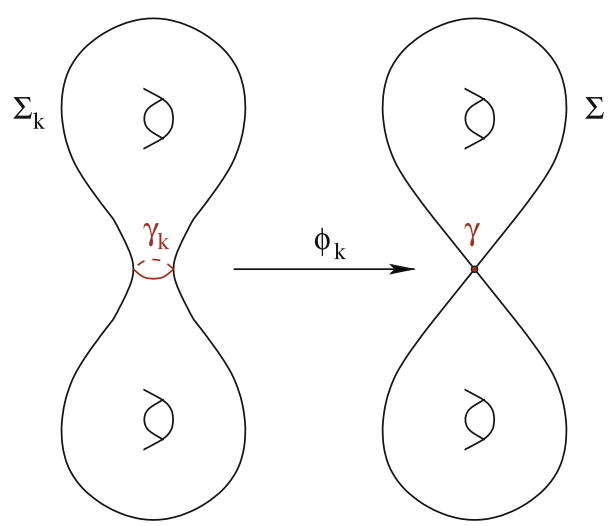

- $\phi$ is a diffeomorphism from $\Sigma^{\prime} \backslash \gamma^{\prime}$ onto $\Sigma \backslash \gamma$, where $\gamma:=\bigcup\left(v \backslash \phi_{*} \nu^{\prime}\right)$.

- $\phi \circ \sigma \mid \operatorname{int}\left(\Sigma_{\gamma^{\prime}}^{\prime}\right)$ extends to a continuous surjective map $\Sigma_{\gamma^{\prime}}^{\prime} \rightarrow \Sigma$ such that the preimage of each nodal point in $\gamma$ is a component of $\partial \Sigma_{\gamma^{\prime}}^{\prime}$ and two boundary components which map under $\sigma$ to the same component of $\gamma^{\prime}$ map to a nodal pair $\{x, y\} \in \gamma$.

Each component of $\gamma^{\prime}$ is called a vanishing cycle of the deformation $\phi$. A sequence $\phi_{k}$ : $\left(\Sigma_{k} \backslash \gamma_{k}, v_{k}\right) \rightarrow(\Sigma, v)$ of $\left(v_{k}, v\right)$-deformations is called monotypic if $\left(\phi_{k}\right)_{*} v_{k}$ is independent of $k$.

Definition 8.3 Let $M$ be a complex manifold. A sequence $\left(\Sigma_{k}, s_{k, *}, v_{k}, j_{k}, v_{k}\right)$ of configurations in $M$ of type $(\mathrm{g}, \mathrm{n}, \mathrm{d})$ is said to converge monotypically to a configuration $\left(\Sigma, s_{*}, v, j, v\right)$ of type $(\mathrm{g}, \mathrm{n}, \mathrm{d})$ iff there is a monotypic sequence $\phi_{k}: \Sigma_{k} \backslash \gamma_{k} \rightarrow \Sigma \backslash \gamma$ of $\left(v_{k}, v\right)$-deformations satisfying the following conditions.

(Marked points)

(Complex structure)

(Map)

(Energy)

For $\mathrm{i}=1, \ldots, \mathrm{n}$ the sequence $\phi_{k}\left(s_{k, \mathrm{i}}\right)$ converges to $s_{\mathrm{i}}$ in $\Sigma$.

The sequence $\left(\phi_{k}\right)_{*} j_{k}$ of complex structures on $\Sigma \backslash \gamma$ converges to $j \mid(\Sigma \backslash \gamma)$ in the $C^{\infty}$ topology.

The sequence $\left(\phi_{k}\right)_{*} v_{k}:=v_{k} \circ \phi_{k}^{-1}$ converges to $v \mid(\Sigma \backslash \gamma)$ in the $C^{\infty}$ topology on $C^{\infty}(\Sigma \backslash \gamma, M)$.

For some (and hence every) pair of Riemannian metrics on $\Sigma$ and $M$ we have

$$
\lim _{\varepsilon \rightarrow 0} \lim _{k \rightarrow \infty} \int_{B_{\varepsilon}(\gamma)}\left|d\left(v_{k} \circ \phi_{k}^{-1}\right)\right|^{2}=0,
$$

where $B_{\varepsilon}(\gamma) \subset \Sigma$ denotes the $\varepsilon$-neighborhood of $\gamma \subset \cup v$.

The sequence $\left(\Sigma_{k}, s_{k, *}, v_{k}, j_{k}, v_{k}\right)$ is said to Gromov converge to $(\Sigma, j, s, v, v)$ if, after discarding finitely many terms, it is the disjoint union of finitely many sequences which converge monotypically to $(\Sigma, s, v, j, v)$.

Theorem 8.4 Let $\left(\Sigma, s_{*}, v, j, v\right)$ be a stable map, $\left(\pi: Q \rightarrow B, S_{*}, H, b_{0}\right)$ be a universal unfolding, $u_{0}: \Sigma \rightarrow Q_{b_{0}}$ be a desingularization with induced structures $s_{*}, v, j$, and $v$ on $\Sigma$, and $\left(\Sigma_{k}, s_{k, *}, v_{k}, j_{k}, v_{k}\right)$ be a sequence of stable maps. Then the following are equivalent.

(i) The sequence $\left(\Sigma_{k}, s_{k, *}, v_{k}, j_{k}, v_{k}\right)$ Gromov converges to $\left(\Sigma, s_{*}, v, j, v\right)$. 
(ii) After discarding finitely many terms, there exist $b_{k} \in B$ and desingularizations $u_{k}: \Sigma_{k} \rightarrow Q_{b_{k}}$ inducing $s_{k, *}, v_{k}, j_{k}$, $v_{k}$ such that $b_{k}$ converges to $b_{0}$.

If (i) holds with a sequence of deformations $\phi_{k}: \Sigma \backslash \gamma_{k} \rightarrow \Sigma$ then the sequence $u_{k}$ in (ii) can be chosen such that $u_{k}\left(\gamma_{k}\right)$ converges to the nodal set in $Q_{b_{0}}$ and $u_{k} \circ \phi_{k}^{-1}: \Sigma \backslash \cup v$ converges to $u_{0} \mid(\Sigma \backslash \cup v)$ in the $C^{\infty}$ topology.

Proof We prove (ii) implies (i). Let $u: \Sigma \rightarrow Q_{b_{0}}$ be a desingularization. Assume that $b_{k}$ converges to $b$ and that $u_{k}: \Sigma_{k} \rightarrow Q_{b_{k}}$ is a sequence of desingularizations inducing ( $s_{k, *}$, $\left.v_{k}, j_{k}, v_{k}\right)$. As in the proof of [4, Theorem 13.6] there are maps $\psi_{b}: Q_{b} \rightarrow Q_{b_{0}}$ and deformations $\phi_{k}: \Sigma_{k} \backslash \gamma_{k} \rightarrow \Sigma$ such that $\psi_{b}$ agrees with a smooth trivialization away from the nodal set, $\psi_{b_{0}}$ is the identity, and

$$
u \circ \phi_{k}=\psi_{b_{k}} \circ u_{k}: \Sigma_{k} \backslash \gamma_{k} \rightarrow Q_{b_{0}} .
$$

Assume w.l.o.g. that the sequence $\phi_{k}$ is monotypic so that there is a subset $\gamma \subset \cup v$ such that $\phi_{k}: \Sigma_{k} \backslash \gamma_{k} \rightarrow \Sigma \backslash \gamma$ is a diffeomorphism. As in [4] the sequence $\phi_{k}\left(s_{k, \mathrm{i}}\right)$ converges to $s_{\mathrm{i}}$ in $\Sigma$ and the sequence $\left(\phi_{k}\right)_{*} j_{k}$ of complex structures on $\Sigma \backslash \gamma$ converges to $j \mid(\Sigma \backslash \gamma)$ in the $C^{\infty}$ topology. Now $\psi_{b_{k}}^{-1} \circ u_{0}=u_{k} \circ \phi_{k}^{-1}$ so

$$
H \circ \psi_{b_{k}}^{-1} \circ u_{0}=H \circ u_{k} \circ \phi_{k}^{-1}=v_{k} \circ \phi_{k}^{-1} \text {. }
$$

Since $\psi_{b_{0}}$ is the identity the left hand side (and hence also $\left.\left(\phi_{k}\right)_{*} v_{k}=v_{k} \circ \phi_{k}^{-1}\right)$ converges to $v_{0} \mid\left(\Sigma_{0} \backslash \gamma\right)$ in the $C^{\infty}$ topology on $C^{\infty}(\Sigma \backslash \gamma, M)$.

We prove (i) implies (ii) under the additional hypothesis that the marked nodal Riemann surface $\left(\Sigma, s_{*}, v, j\right)$ is stable. By the uniqueness of universal unfoldings we may asssume that $\left(\pi, S_{*}, H, b_{0}\right)$ is given by (72). By assumption, the sequence $\left(\Sigma_{k}, s_{k, *}, v_{k}, j_{k}\right)$ obtained by discarding the maps $v_{k}$ consists of stable marked nodal Riemann surfaces and it DM-converges to $\left(\Sigma, s_{*}, v, j\right)$ as in [4, Definition 13.3]. Hence Theorem 13.6 in [4] asserts that there exists a sequence $a_{k} \in A$ converging to $a_{0}$ and, for sufficiently large $k$, desingularizations $w_{k}: \Sigma_{k} \rightarrow P_{a_{k}}$ inducing the structures $s_{k, *}, v_{k}, j_{k}$ on $\Sigma_{k}$. By [4, Remark 13.9], the desingularizations $w_{k}$ can be chosen such that the sequence

$$
w_{k} \circ \phi_{k}^{-1}: \Sigma \backslash \cup v \rightarrow P
$$

converges to $w_{0}$ in the $C^{\infty}$ topology. Define $h_{k}: P_{a_{k}} \rightarrow M$ and $h_{0}: P_{a_{0}} \rightarrow M$ by

$$
h_{k} \circ w_{k}:=v_{k}, \quad h_{0} \circ w_{0}:=v_{0} .
$$

Since $w_{k} \circ \phi_{k}^{-1}$ converges to $w_{0}$, the sequence $\phi_{k} \circ w_{k}^{-1} \circ \rho_{a_{k}}^{-1}$ (with $\rho$ as in 5.10) converges to $w_{0}^{-1}$ in the $C^{\infty}$ topology on $\Omega=P_{a_{0}}^{\prime \prime}$. This implies that the sequence

$$
h_{k} \circ \rho_{a_{k}}^{-1}=\left(v_{k} \circ \phi_{k}^{-1}\right) \circ\left(\phi_{k} \circ w_{k}^{-1} \circ \rho_{a_{k}}^{-1}\right)
$$

converges to $v_{0} \circ w_{0}^{-1}=h_{0}$ in the $C^{\infty}$ topology on $\Omega$. By definition of $\mathcal{V}^{\prime \prime}$, this implies

$$
b_{k}:=\left(a_{k}, \beta_{k}\right) \in \mathcal{V}^{\prime} \cap \mathcal{V}^{\prime \prime}=B, \quad \beta_{k}:=h_{k} \mid \Gamma_{a_{k}} \in \mathcal{V}_{a_{k}}^{\prime \prime}
$$

for $k$ sufficiently large. Here we have also used the fact that $h_{k} \mid P_{a_{k}}^{\prime}$ takes values in $\mathcal{V}^{\prime}$ for large $k$, by the (Energy) axiom and the standard compactness arguments for pseudoholomorphic curves (see [2, Chap. 4]). Since $a_{k}$ converges to $a_{0}$ and $\beta_{k} \circ \rho_{a_{k}}^{-1} \mid \partial \Omega$ converges to $\beta_{0}:=h_{0}\left|\Gamma_{a_{0}}=h_{0}\right| \partial \Omega$, we deduce that $b_{k}$ converges to $b_{0}:=\left(a_{0}, \beta_{0}\right)$. Thus we have proved that (i) implies (ii) under the assumption that the marked nodal Riemann surface $\left(\Sigma, s_{*}, v, j\right)$ is stable. 
We prove (i) implies (ii) in general. Suppose the sequence $\left(\Sigma_{k}, s_{k, *}, v_{k}, j_{k}, v_{k}\right)$ Gromov converges to $\left(\Sigma, s_{*}, v, j, v\right)$ and the underlying marked nodal Riemann surface $\left(\Sigma, s_{*}, v, j\right)$ is not stable. Then we can add marked points to $\Sigma_{k}$ and $\Sigma$ such that the resulting sequence still Gromov converges and the augmented marked nodal Riemann surface $\left(\Sigma, s_{*}, t_{*}, v, j\right)$ is stable. By what we have already proved, the augmented sequence $\left(\Sigma_{k}, s_{k, *}, t_{k, *}, v_{k}, j_{k}, v_{k}\right)$ satisfies (ii). Let $\left(\pi_{A}: P \rightarrow A, R_{*}, T_{*}, H_{A}, a_{0}\right)$ be a universal unfolding of the augmented stable map. Removing the additional sections $T_{*}$ results in an unfolding that is no longer universal but, by definition of universal, admits a morphism to ( $\pi: Q \rightarrow B, S_{*}, H, b_{0}$ ). Hence the original sequence $\left(\Sigma_{k}, s_{k, *}, v_{k}, j_{k}, v_{k}\right)$ also satisfies (ii). This proves the theorem.

\section{Concluding remarks}

It would be interesting to know to what extent the techniques developed in this paper extend to the nonintegrable case. Since the linearized Cauchy-Riemann operators $D_{v}$ are not complex linear in this case the resulting moduli space will at best be a smooth (not a complex) orbifold. In the definition of a universal unfolding we can at most expect the existence of a smooth morphism $(\Phi, \phi): \pi_{A} \rightarrow \pi_{B}$. An analogue of the universal unfolding theorem (Theorem 3.6) for the nonintegrable case will depend on an answer to the following question.

Given an almost complex structure $J$ on $\mathbb{R}^{2 \mathrm{~m}}$ and a complex number $z \in \operatorname{int}(\mathbb{D})$ define the set

$$
\mathcal{N}_{z}:=\left\{\begin{array}{l|l}
(\xi, \eta, z) \in H^{s}\left(S^{1}, \mathbb{R}^{2 \mathrm{~m}}\right)^{2} & \begin{array}{l}
\exists \text { a } J \text {-holomorphic map } \\
v: N_{z} \rightarrow \mathbb{R}^{2 \mathrm{~m}} \text { in } H^{s+1 / 2} \\
\text { s.t. } \xi=v \circ \iota_{1, z}, \eta=v \circ \iota_{2, z}
\end{array}
\end{array}\right\}
$$

where $N_{z}$ is as in 5.13. It is easy to prove that this set is a smooth submanifold of $H^{s}\left(S^{1}, \mathbb{R}^{2 \mathrm{~m}}\right) \times H^{s}\left(S^{1}, \mathbb{R}^{2 \mathrm{~m}}\right)$ for every $z$. A natural question to ask is if the disjoint union

$$
\mathcal{N}:=\bigcup_{z \in \operatorname{int}(\mathbb{D})}\{z\} \times \mathcal{N}_{z}
$$

is a smooth submanifold of $\operatorname{int}(\mathbb{D}) \times H^{s}\left(S^{1}, \mathbb{R}^{2 \mathrm{~m}}\right) \times H^{s}\left(S^{1}, \mathbb{R}^{2 \mathrm{~m}}\right)$. In Lemma 5.14 this was proved in the integrable case. However, we have examples of finite dimensional analogues where this fails. On the other hand, we expect that the Hadamard proof of the unstable manifold theorem carries over to the infinite dimensional setting and shows that the $\mathcal{N}_{z}$ form a continuous family of smooth submanifolds. This would give an alternative approach to the gluing theorem for pseudoholomorphic curves. Moreover, one could then carry over the techniques of this paper to prove that, in the almost complex case, the regular stable maps form a $C^{0}$ orbifold.

\section{References}

1. Fulton, W., Pandharipande, R.: Notes on stable maps and quantum cohomology. In: Algebraic GeometrySanta uz 1995 ed. Kollar, Lazersfeld, Morrison. Proc. Symp. Pure Math. 62, 45-96 (1997)

2. McDuff, D., Salamon, D.A.: J-holomorphic Curves and Symplectic Topology. AMS Colloquium Publications, Providence (2004)

3. Milnor, J.: Topology from the Differential Viewpoint. Princeton University Press, Princeton (1965)

4. Robbin, J.W., Salamon, D.A.: A construction of the Deligne-Mumford orbifold. J. Eur. Math. Soc. 8, 611-699 (2004). Corrigendum, July 2007, to appear in JEMS.

5. Ruan, Y., Tian, G.: A mathematical theory of quantum cohomology. J. Differ. Geom. 42, 259-367 (1995) 